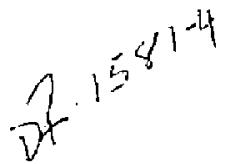

\title{
A COHPARISON OF VARIOUS NLTE CODES IN COMPUTING THE CHARGE-STATE POPULATIONS OF AN ARGON PLASMA
}

\author{
Sam R. Stone and Jon C. Weisheit \\ Physics Department \\ Lawrence Livermore National Laboratory \\ Livermore, Callfornia 94550
}

November 1984
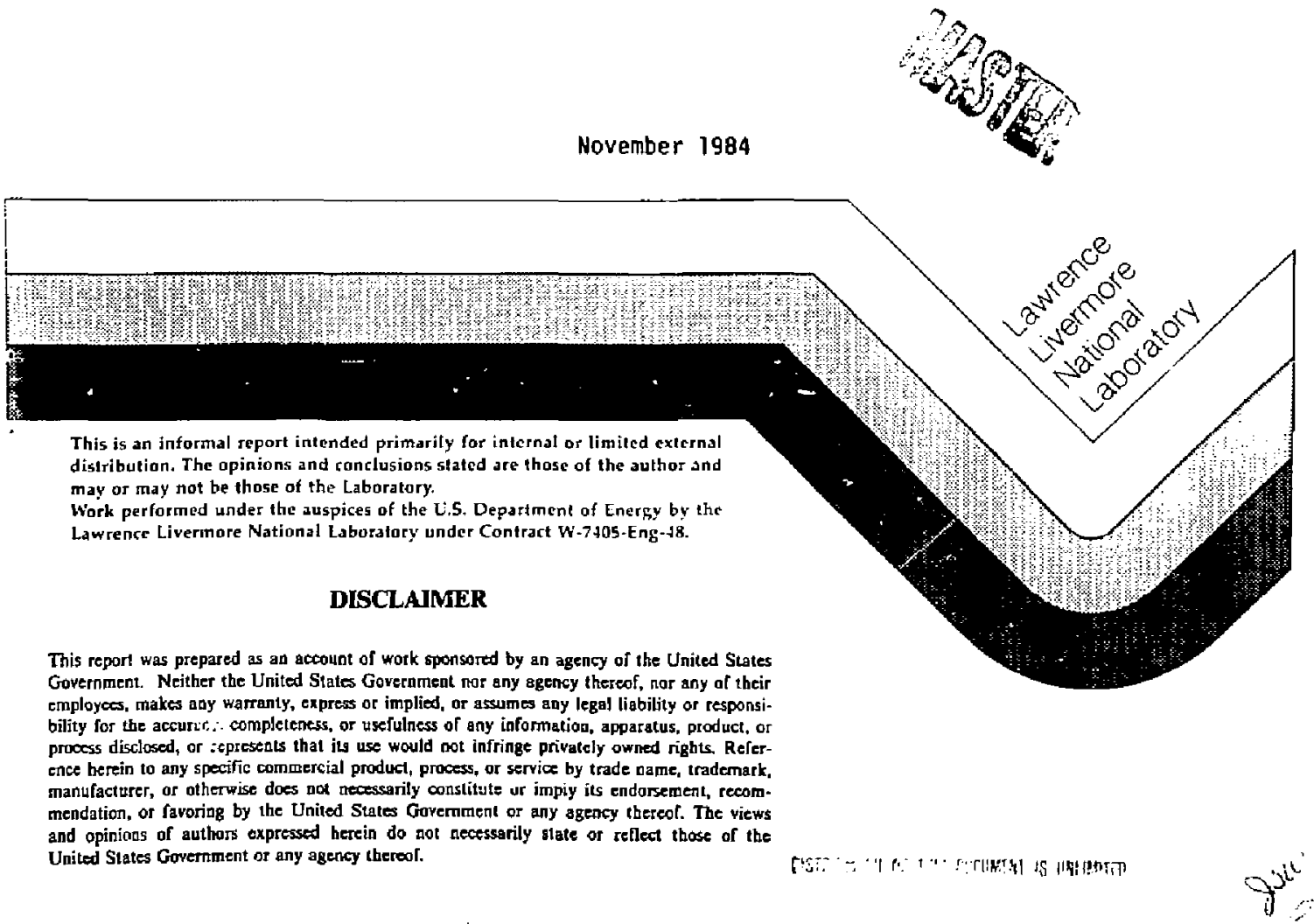


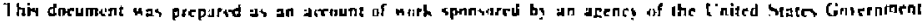

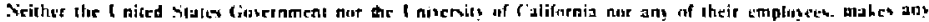

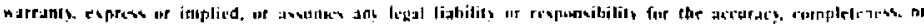

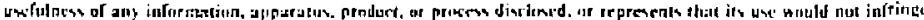

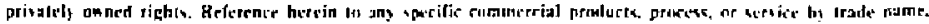

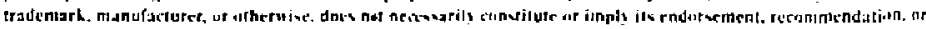

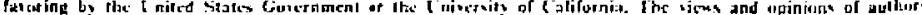

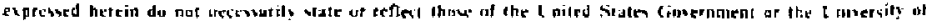

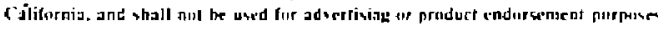

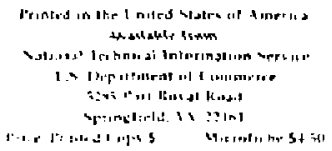

\begin{tabular}{|c|c|c|c|c|}
\hline 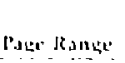 & $\begin{array}{l}\text { Iromentic } \\
\text { 'tuic }\end{array}$ & 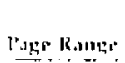 & 1). & $\begin{array}{l}\text { mentis } \\
\text { Prisne }\end{array}$ \\
\hline $1: 011-62=$ & $5>00$ & $326-750$ & 5 & $26.26 !$ \\
\hline (Isn-3is & K. $\$ 0$ & 351.575 & & 28.116 \\
\hline 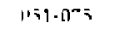 & In nil & $\vec{x} \boldsymbol{x}-\|1\|$ & & 24.711 \\
\hline${ }^{2}+n-1011$ & {$[1.3]$} & $f(1)-i 2 x_{1}$ & & 31.0111 \\
\hline ר-1.111 & \.3.1W & $+27-4513$ & & 32.511 \\
\hline $1 \leq n \cdot 1=1$ & 11.50 & $451-475$ & & 34.041 \\
\hline $1: 1-1: 7$ & 16.100 & & & 33.710 \\
\hline $15 n \cdot 2(n)$ & 1:.711 & $5(11-525$ & & 37 \\
\hline $201-\because 25$ & 14.011 & $520-5 \div 1$ & & 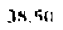 \\
\hline $2 \geq t-2)$ & 20.51 & $551-575$ & & $90.010]$ \\
\hline $241-2-5$ & 22.611 & ה) & & +1.50 \\
\hline $2 \div 6-300$ & 23.50 & $601-u p^{1}$ & & \\
\hline 311 [ - 1ב5 & 25.40 & & & \\
\hline
\end{tabular}

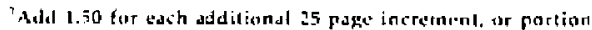
thereth fron hall pagei up. 
A COMPARISON OF VARIOUS NLTE CODES IN COMPUTIHG

THE CHARgE-STATE POPULATIONS OF AN ARgON PLASMA

Sam R. Stone and Jon C. Weisheit

Physics Department, Lawrence Livermore National Laboratory

Livermore, California 94550

ABSTRACT

A comparison ameng nine computer codes shows surprisingly large differences where it had been believed that the theory was well understood. Each code treats an argon plasma, optically thin and with no external photon flux; temperatures vary around $1 \mathrm{keV}$ and ion densities vary from $6 \times 10^{17} \mathrm{~cm}^{-3}$ to $6 \times 10^{21} \mathrm{~cm}^{-3}$. At these condtions most ions have three or fewer bound electrons. The calcuiated populations of $0-, 1-, 2-$, and $3-e l e c t r o n$ ions differ from code to code by typical factors of 2 , in some cases by factors greater than 300 . These differences depend as sensitively on how many Rydberg states a code allows as they do on variations among computed collision rates. 


\section{INTROOUETION}

For some years now computer codes have existed that simulate a plasma in steady-state even if, or especially if, the plasma is not in local thermodynamic equilibrium. For almost that long the people who use these non-local-thermodynamic-equflibrium codes (NLTE codes) have worried that the codes agree with each other less than expected, even for plasma conditions where the physics is believed to be well understood.

One purpase of this paper is to compare results from ten NLTE codes. We concentrate on what populations the codes predict in each ionic charge-state of a particular plasma. We find surprising differences between codes, ranging from a few percent to more than two orders of magnitude. A second purpose is to infer why the codes disagree. The populations are found to depend sensitively on how many energy levels are considered for each ionic charge-state; they seem less sensitive to the calculated rate coefficients than we expected.

We do not pursue here a third possible purpose, to determine which codes are most correct. Comparison to experiment is explicitly not included, and we have tried hard to mistrust "physics by concensus." Indeed the codes considered tend to agree in groups of two or three codes and to diagree between groups. Still we claim neither accuracy nor inaccuracy for any code.

A standard test problem was used to compare the NLTE codes: a one-element argon plasma with its modest $z=18$, opically thin in order that all radiative absorption would be negligible. Densities were considered from near the low density coronal limit up to about $10^{21}$ electrons/cc, where $Z$-pinch experiments might provide some data, and even higher. Temperatures of ions and of electrons were taken equal and hot enough that most of the ionic 
charge-states would have three or fewer bound electrons, which for argon means hotter than about $300 \mathrm{ev}$.

In Section II, the results from the NLTE codes are presented and compared. wine of the codes compute the same standard argon test plasma, and populations of the $0-, 1-, 2-$, and 3-electron ions are graphed in detail. Also graphed are the ratios of rates, the net effective fonization/ recombination, for the charge-states computed. For a tenth code the only results available are for an oxygen plasma, and we compare it to oxygen equilibria computed by two of the other nine codes.

In Section III we show how much the populations change if the various rate coefficients are reduced or if the number of energy levels or the fine structure is altered. This is done for calculations by a single code; thereby we get a feel for the relative importance of a change not complicated by code-to-ccis: comparison.

In Section IV we summarize the results. An Appendix discusses at length the lowering of the continuum in plasmas. 


\section{COMPARISON AMONG THE COMPUTER CODES}

In all we compare calculations by ten different NLTE codes. The codes are listed in Table 1. Of these codes nine compute the ion populations of various charge-states in the same optically thin argon plasma. Seven of the nine are at Lawrence Livermore National Laboratory (LLNL). NRL is the Naval Research Laboratory, and PPL is the Plasma Physics Laboratory at Princeton University. One other code, here called IPP, computes equilibria in an oxygen plasma instead and is compared to LINEZ and RATION only: IPP is the Institute of Plasma Physics at Nagoya University. Japan.

TABLE 1.

List of the NLTE codes corapared. The rame used herein is different in some cases from how the code is known away from Lawrence Livermore National Laboratory (LLNL).

\begin{tabular}{rlc}
\hline COOE & Principal Authors/Institution & Reference \\
YTL & Y. T. Lee/LLML & 1 \\
XSN & W. Lokke, W. Grasberger, et al./LLNL. & 2 \\
XRASER & P. L. Hagelstein, W. L. Morgan, et al. ILLNL & 3 \\
RATION & R. W. Lee, et al./LLNL & 4 \\
PPL & R. Hulse/Princeton PPL & 5 \\
NRL & D. Duston/MRL, Washington, D.C. & 6 \\
NEBULA & C. B. Tarter/LLNL \\
LINEZ & J. C. Weisheit/LLNL \\
IPP & T. Fujimoto, T. Kato/Institute of Plasma Physics, Nagoja & 9 \\
FOREST & W. Grasberger/LLNL
\end{tabular}




\section{A. What is the Standard Argon Problem? \\ The test case we chose for comparing the NLTE codes is one that was}

agreed on ax LLNL in 1981 by $R$. J. Fortner and others. That choice was the single-element argon plasma, optically thin and with negligible external photoin flux. The particle temperatures were around 1 keV and the density was at or near $6 \times 10^{19}$ ions/cc.

There were severa? reasons for this choice. Livermore's $z$-pinch experiment $2 A P P$ could achieve those conditions in argon. The populations of lanic charge-states were expected to be dominated by He-like Ar XVII, so the developers of the various codes were confident that most atomic data for this case would be known fairly accurately. At those conditions the rate of dielectronic recombination was estimated to be fairly small, and in 1981 only two codes included that rate.

\section{B. What Populations do the codes Compute?}

Each code was asked to calculate Ar-ion populations in a range of temperatures $T$ and for one or more plasma densities. How many of the total ions are He-like Ar XVII. for example, varies with temperature $T$ and with ion density $n_{j}$ : when $n_{j}=6 \times 10^{19}$ ions/ce and $T=1 \mathrm{keV}$ then the He-like population is around 4 or $5 \times 10^{19} \mathrm{~cm}^{-3}$, and its relative population or population fraction is some 60 to $90 \%$.

An example of how populations are plotted is shown in Fig. 1 . In this example the cede YTL computes populations of Li-like, He-like, H-like, and bare ions. The density of all electrons, bound and unbound, is $10^{21} \mathrm{~cm}^{-3}$. Since $z=18, n_{i}=5.556 \times 10^{19}$ ions/cc, and the mass density is $3.685 x$ $10^{-3} \mathrm{~g} / \mathrm{cc}$. The figure shows population fractions for temperatures $r$ from 
100 to $10,000 \mathrm{ev}$. Not shown are the populations of charge-states with more than three electrons, such as Be-like, 8-7ike, etc.

Figures 2, 3, and 4 display the calculated populations in a way that al lows the reader to choose his own zomparisons. Using the same format as in Fig. I there is a separate transparent overlay for the populations computed by each code. Figure 2 is the most complete series; it shows the results in the optically thin argon plasma at the standard density $n_{j} \simeq 5.6 \times 10^{19} \mathrm{ion} / \mathrm{cc}$. The cades compared are RATION, XRASER, LINEZ, YTL, FOREST, and NRL.

In Fig. 2 we see that each charge-state population has its own characteristic temperature range, with fairly narrow peaks in Li-like and H-like and a broad maximum in He-like. The Li-like peak is at 150 eV (YTL) or at $210 \mathrm{eV}$ (RATION). LINEZ sees no Li-like peak because it computes only the four charge-states shown; all ions not having 0,1 , or 2 bound electrons are assumed to be Li-like. The He-like maximum is broadest and hottest with XRASER, coolest with LINEZ and YTL. The H-like peak is high and cool (LINEZ) or low and hot (XRASER). All codes eventually reach the high temperature region where most ions are bare. A striking feature of figure 2 is the dissimilarity among codes. At $1 \mathrm{keV}, \mathrm{Li}-1 \mathrm{k}$ e populations disagree by a factor of 6.3 and He-like populations by a factor of 1.6; $\mathrm{H}-1$ ike and Dare populations vary more than factors of 30 .

Figure 3 compares XRASER, RATION, and IINEZ at an ion density $n_{i}=$ $5.6 \times 10^{17}$ ion/cc, two orders of magnitude less dense than the standard problem in Fig. 2. This much lower density approaches the coronal limit, and so Fig. 3 aiso compares the data from two coronal codes, NEBULA and PPL. In Fig. 3 we see the Lf-like peak is either high and cool (XRASER, RATION), or low and hat $(\mathrm{PPL})$, but the codes agree on Li-like ions to within a factor of 2.5 between 350 and $2,000 \mathrm{eV}$. Similarly the codes agree on He-like ians 
across the maximum above $500 \mathrm{eV}$, except that XRASER shows a much broader maximum above $1,000 \mathrm{eV}$ than the other codes. The :i-like peaks are fairly similar except that XRASER sees much less H-like argon below 9,000 eV. All but XRASER agree quite well on bare populations. In general the two coronal codes (NEBULA, PPL) agree very closely.

Figure 4 compares KRASER, RATION, and LINEZ at an ion denisty $n_{i}=$ $5.6 \times 10^{21}$ ion/cc. two orders of magnitude more dense than the standard problem. In Fig. 4 the agreement is generally within a factor of 2 between RATION and LINEZ, while XRASER differs Erom them by factors of 2 to 16 . The differences represent relatively more ionization by LINE2. less by XRASER.

Figures 2, 3, and 4 taken together also allow a comparison of how populations vary with density as computed by the three codes XRASER, RATION, and LINEZ. RATION shows a smooth progression of increasing ionization as density increases four orders of magnitude. The trend of ionization with LINEZ is less regular but very similar to that by RATION. XRASER ionizes with density even more irregulariy, reversing the process with Li-like ions. In comparing Figures 2 and 3 there is surprising agreement between NRL at $5.6 \times 10^{19}$ ions/ce and the two coronal codes NEBULA and PPL. NRL and PPL agree to within $40 \%$ at temperatures above $500 \mathrm{eV}$.

Another series of plots compares the average-atom code XSN to LINEZ at three different densities. Any average-atom code calculates fractional numbers of electrons in each electronic shell (principal quantum number $n=1$, 2, 3, etc.) instead of computing populations of charge-state lons (bare. H-like, He-like, etc.). For that reason Figs, 5.7, and 9 show for three different densities the numbers of electrons in the lowest shells of the average atom. And at the same three densities Figs. 6, 8 , ana 10 show how the charge $\bar{z}$ on the average atom varies with temperature. 
In Figs. 5-10 we sef that XSN and LINEZ agree poorly at low density and more closely at high definsity. At the near-coronal $5.6 \times 10^{17}$ ions/cc of fig. 5 the average-atom $X S N$ depopulates $i t s ~ L-$ and M-shells abruptly as temperature increases from 1300 to $1600 \mathrm{eV}$. In the $\bar{z}$ plots of Figs. 6, 8. and 10 LINEZ shows an average ionization increasing with density above $800 \mathrm{eV}$, but below that temperature it sees its largest $\vec{z}$ at the intermediate density $5.6 \times 10^{19}$ ions/CC. (Because LINEZ assumes all charge-states have $0,1,2$, or 3 bound electrons only. its results are valid where Lf-like ions are a small minarity; that is, above about $300 \mathrm{eV}$ for these Ar plasmas.)

c. What Ionization/Recombination Ratios do the Codes Compute?

Another way to compare the different NLTÉ codes is to examine the ratio of net ionization/recombination that each code computes for the ioric charge-states. For each ith charge state, whose fon has $i$ bound electrons, we define:

$n_{j}=$ no. density of ith fonic charge-state

$I_{i}=$ rate coefficient of net ionization out of the 1 th state

$\mathbf{R}_{\mathbf{i}}=$ rate coefficient of net recombination into the ith state

$n_{e}=$ no. density of unbound electrons

Steady-state conservation of total ions means that

$$
\begin{array}{r}
n_{1} n_{e} I_{1}=n_{0} n_{e}^{R_{1}} \\
n_{0} n_{e} R_{1}+n_{2} n_{e} I_{2}=n_{1} n_{e} I_{1}+n_{1} n_{e} R_{2} \\
n_{1} n_{e} R_{2}+n_{3} n_{e} I_{3}+n_{2} n_{e} I_{2}+n_{2} n_{e} R_{3} \\
\text { etc. for higher } 1 .
\end{array}
$$


This set of equations yields a ratio for each $t$-electron ion:

$$
\frac{I_{i}}{R_{i}}=\frac{n_{i-1}}{n_{i}}
$$

Therefore comparing the ratios of net fonization/recombination rates is equivalent to comraring ratios of populations rather than the populations themselves. These ratios of rates are particularly relevant to inaiyzing spectra that involve lines from more than one charge-state. Notice however that $I_{i}$ and $R_{j}$ defined here are not the collisional-radiative rates of Bates et al. " those rates are defined by the pupulation ratios of successive ground-state ions, and at higher densities than corcnal. substantial fonic populations reside in the excited levels.

Figure 11 compares the ratios of net ionization to net recombinatian in the same way that Fig. 2 compares populations. There is a separate transparint overlay for the ionization/recombination : $f$ each code compared. And as in Fig. 2, Fig. 11 treats the argon plasma ar the standard density $5.6 \times 10^{19}$ ion/cc. In Fig. 11 we see a wide scatter in ratios of ionization/ recombination rates, which reflects the scatter in populations shown by Fig. 2. For Li-like inns YTL ionizes most and NRL least: they differ hy factors of 5 to 6 . For He-like ions LINEZ fonizes most and XRASER least; differences are factors of 15 to 50 . The H-like rates are much closer. XRASER and NRL are similar, while RATION, LINF., YTI, and FOREST agree quite closely; the two groups differ by a factor of 2 . XRASER is the only cadf for which the H-like jons show a higher ratio of rates than the He-like (above 1,100 eV).

Figure 12 compares the net lonization/recombination ratios at the much lower density $5.6 \times 10^{17}$ ion/cc. two orders of magni*ude less dense than the 
standard problem. Again as in Fig. 3, because this density approaches the coronal limit, Fig. 12 also includes data from the coronal codes NEGULA and PPL. In Fig. 12 the codes agree somewhat more closely but again with striking exceptions. For Li-like ions XRASER and RATION give ratios almost equal to each other at all temperatures; they differ by less then 15\%. LINEZ agrees with XRASER-RATION up to $600 \mathrm{eV}$, then ionizes less Li-like ions by as much as a factor of 3.5. NEBULA has the lowest Li-1ike ratio, but NEBULA and PPL agree with LINEZ at $3,000 \mathrm{eV}$, where their ratio is a third that of XRASER and RATION. With He-like tons RATION. NEBULA, and PPL agree, while LINEZ's ratio is up to 3 times greater and XRASER's is 2 orders of magnitude less. For H-like ions all codes agree to within $30 x$ except XRASER, whose ratio is less by factors of 1.4 to 2.8 .

Figure 13 compares the net iorization/recombination ratio for the higher density $5.6 \times 10^{21}$ ion/cc, two orders at magniture more dense than the standard problem. In Fig. 13 the three codes differ more consistently: XRASER's ratio is least and LINEZ's is biggest, except that RATION fonizes relatively more Li-like ions.

Figures 11, 12, and 13 taken together allow a comparison of how the net ionization/recombination ratlo varies with density as computed by the three codes XRASER, RATION, and LINEZ. RATION varies less than $30 X$ in the Li-like ratio as the density varies four oroiers of magnitude, while the de-like and H-like ratios increase more substantially with density. XRASER and LINEZ both give their biggest ratio of rates at the intermediate density and their smallest ratio at the high density. With XRASER the He-like ratio varies as the square root of density. which is a much stronger dependency than RATION and LINEL show. XRASER's He-like ratio even falls below its $\mathrm{H}-1$ ike ratio at 
densities beiow about $10^{20}$ ions/cc. A11 three codes show a similar pattern of $\mathrm{H}$-like ratio increasing with density.

Recently the proprietors of the IPp code published ${ }^{9}$ rates computed for an opticaliy thin oxygen plasma. Their rates are the collisianal-radiative rates defined by bates et 1 1. " which are the net fonfzation out of and net recombination into just the ground state of He-like aVII. For comparison it was convenient to compute those same rates using the codes RATION and LINEZ.

Figure 14 compares this ratio of [net ionization out of ]/[net recombination intol the ground state of He-like OVII as computed by IPP, RATION, and LINEZ. The ratio is lowest for IPP at all densities. The RAIION ratio shows a dip at $10^{18}$ electrons/cc. LINEZ peaks broadly between $10^{17}$ and $10^{21}$ electrons/cc. Note that the IPP and LiNEZ results exhfbjt similar behaviors of the ratio, although they differ by factors of 1.3 to 3.8 . 


\section{WHAT MAKES THE DIFFERENCE BETWEEN COOES?}

We wish we knew which NLTE code is "best" in the sense of both convenience of computation and agreement with experiment. Lacking that assurance we considered fleetingly trying to explain which features of the codes compared in section II cause the disagreements. And then, declining to dissect someone else's code, we settled instead upon the more modest goal of this section III, which is to look at how big a difference it makes in a single code if we change a raie or restructure the excited states.

Here then we compare not the codes but the relative effect of a cading change: What happens to charge-state populations when we vary the computed rates of excitation/de-excitation or of ionization/recombination? What happens if we permit different numbers of excited levels in eacli ionic charge-state? We use a single vehicle, the computer code LINE, because it is the most convenient code for us to manipulate.

In this one-code comparison of physics effects, certain featur as are not studied because they are common to all the NLTE codes. ror example, every code uses similar energy eigenvalues and Einstein A-values for radiative transitions. Also the test problem is an optically thin argon plasma, so the different codes do not disagree because of their treatment of radiative absorption.

A. Effect of Rate Coefficients on the Charge-State Populations The various methods in use for computing the rate coefficients were expected to be fairly accurate, especially for the simple 1-and 
2-electron fons. For this reason we made only modest changes to the rate coefficients in our one-code comparison of physics effects. What this did to charge-state populations was modest as well, as this sub-section shows.

1. Rates of excitation/de-excitation due to collisions by electrons

To exhfbit the sensitivity of charge-state populations to the electroncollisional rates of excitation/de-excitation, the rates were arbitrarily mutiplied by 0.5 in all ion charge-states, and the results were compared to a normal calculation. Since excitation and de-excitation are connected through detajled balance, both rates were multipled by the same factor.

Figure 15 compares the charge-state populations in argon with and without the half-strength rates of electron-collisional excitation/de-excitation. The effect of the half-strength rates is to shift the charge-state populations toward less ionization; significant effects are 1 ess than a factor of 1.9 in populations. This change is smaller than many of the differences shown in Fig. 2 between various computer codes.

2. Rates of ionization/recombination due to collisions by electrons Just as for excitation/de-excitation considered above, the rate of fonization between charge states was arbitrarily multiplied by 0.5 . The code LINEZ computed electron-collisional ionization using a Jacobs-Lotz formula. ${ }^{14}$ Again the same multiplication by 0.5 applied also to electron-collisional 3-bady recombination through detajled balance,

As an example, we computed rate coefficients for ianization from $\mathrm{H}-1$ ike and He-like Ar ions at temperatures from 300 to $3,000 \mathrm{eV}$. Four widely used formulas were compared: Thamson, 12 Lotz, 13 Jacobs, 14 and Younger. 15 Ionization rates from ground states agreed to within a factor of 1.5 . 
Figure 16 compares the charge-state populations in argon with and without the change to electron-collisional ionization/recombination. The effect of the half-strength rates is to decrease fonization, but with significance only for temperatures hotter than about 2,000 eV. Even there the effects are less than a factor of 1.5 in populations. As with Fig. 15, Fig. 16 shows that the change in populations is smaller than many of the code-to-code differences shown in Fig. 2 .

\section{Rates of dielectronic recombination}

Dielectronic recombination (DR) was sometimes mentioned as a possible reason why one code might differ from another. With the argon test problem DR was not expected to be significant relative to radiative recombination. To confirm this the $O R$ rate was set to zero in LIHEZ.

Figure 17 compares the charge-state populations with and without DR. Only He-like and Li-like populations are affected significantly, and on iy at temperatures hotter than about $1,500 \mathrm{eV}$. Mere again the effects are less than a factor of 1.5 in He-like populations, and they aris smalier than some of the code-to-code differences of Fig. 2.

4. Rates of excitation/de-excitation due to ion collisions

One otter process that can affect the charge-state populations is ion-ion collisions. LINEZ normally includes ion-collisional cr.itation/de -excitation, and so a run was made with these rates set to zero.

Figure 18 compares the charge-state populations with and without ion-collisional excitation/de-excitation. The difference is so small that it is hard to see in Figure 18, about $1 \%$ at $1 \mathrm{keV}$ and as much as $17 \%$ (He-like fons) at $101: 1$. 


\section{Effect of Level Structure on the Charge-State Populations.}

It is hard to separate two of the features of how a code models its energy levels: continuum lowering and number of excited energy levels. One approach is to caiculate the lowering first, then retain all of the levels for which the binding energy is greater than the lowering. Another approach is to compute continuum lowering, but for computational economy keep only a fixed number of energy levels, say 5 or 7 , which is fewer than the number allowed by lowering the continuum. LINEZ can do it efther way; but its preferred method (cf. Bates et al. ${ }^{11}$ ) is to keep all levels for which the lifetime in the plasma exceeds one Bohr orbital period, then lower the continum to the lowest lying level not kept. The first part of the Appendix discusses some of the ways to compute a lowered continuum, such as the widely used stewart-Pyatt formula. The second part of the Appendix treats the Bates et al. ${ }^{11}$ and other level-limiting schemes.

1. Number of energy levels per charge-state

A normal LINEZ run was compared to LINEZ with the principal quantum number $n_{\operatorname{tax}}$ of the uppermost energy level set arbitrarity at 10 and 5. . A11 three runs treated argon at $10^{21}$ electron/cc and temperatures up to 10 key. In the normal run the code retained all energy levels for which the level lifetime exceeded the Bohr orbitai period, and the continumm was lowered to the lowest lying level not retained. The numbers $n_{\text {max }}$ of levels per chargestate varied from 13 ( $H-1$ ike ArXVIII at 600 to $1,500 \mathrm{eV}$ ) to 8 (Li-like

* The choices 10 and 5 were not entirely arbitrary. Other versions of YTL, NRL. RATION, and XRASER had set $n_{\max }=5$. However, those versions were not used in the present study. The choices for $n_{\max }$ that each code used in this study are discussed below and tubulated in figs. 21 and 22. 
ArXVI at 8 to $10 \mathrm{keV}$ ). In the runs with arbitrarily pre-set numbers of levels the Stewart-Pyatt algorithn lowered the continuum.

Figu:e 19 compares normal LINEZ to the arbitrary choice of $n_{\text {max }}=10$. The difference in populations is quite small, usually less than $10 \%$.

Figure 20 compares normal LINEZ to the arbitrary choice of $n_{\max }=5$. Now the difference in populations is large, typlcally by factors of 1.25 to over 2.8. The $H-1 j k e$ peak and the bare populations decrease and shift to hotter temperatures, while the He-like and Li-like populations increase above $1,000 \mathrm{eV}$. The choice $n_{\max }=5$ fails to tonize as much as the normal run.

An intriguing possibility is suggested by comparing fig. 20 to fig. 2. Limiting $\pi_{\max }=5$ in Fig. 20 has the effect of shifting LINEZ populations in the direction of every other code's fopulations shown in fig. 2 . The agreement is especially good between LINEZ limited to $n_{\max }=5$ and nomal RATION (above $1,500 \mathrm{eV}$ ). YTL (above is0 ev!). and FOREST (one temperature point available at 1,000 ev). Just possibly a major cause of disagreement among codes is the number of energy levels allowed per charge-state.

To examine this possibility we ran LINEZ with the uppermost principal quantum number $n_{\text {max }}$ fixed at all values 2 through 15 .

Figure 21 shows how charge-state populations vary with $n_{\max }$. At the single temperature/density of the standard Ar problem, populations change by factors of 1.3 to 4.5 , and the direction is generally toward increasing tonization with increasing $\pi_{\max }$. There is a "knee" in each population curve at an $n_{\max }$ of about 10 abcve which the populations change fairly little from what seem to be their asymptotic values.

A transparent overlay to Fig. 21 displays the same populations as computed by each code from Fig. 2. Here the vertical axis is the same as in Fig. 21, but horizontally the codes are simply ordered by their increasing 
effective $n_{\max }$, which is different than the linear $n_{\max }$ scale of Fig. 21. At the bottom of the overiay each code is named (and color-coded with its data), and the $n_{\text {max }}$ used by each code is tabulated. It is seen that $n_{\max }$ alone cannot explain the code-to-code discrepancies. However, the variations with $n_{m: x}$ have the same qualitative shape in Fig. 21 and in its overlay. Figure 22 displays the effect of $n_{\text {max }}$ on ratios of net tonization/ recombination. It uses the same data as in Fig. 21 , and the same "knee" appears at about $n_{\max }=10$. The ratio for $H$-ilke ionization/recombination is nearly unaffected by $n_{\max }(22 \%)$. While the He-like ratio grows with $n_{\max }$ by a factor of 5.5. Again there is a transparent overlay to fig. 22 which shows the same ratios as computed by each code from figs, 2 and 11 . The vertical axis is the same as in Fig. 22 but horizontally the codes in the overlay are simply ordered by their increasing effective $n_{\text {max }}$. just as in the overlay of Fig. 21 . Again it is seen that $n_{\text {max }}$ aione cannot explain the code-to-code differences but the trend of ratio vs $n_{\max }$ is the same in fig. 22 and in its overlay.

2. Fine structure in He-like energy levels

The excited energy levels of an ion are split slightly by spin-orbit coupling. electrostatic shielding, and other perturbations to the central field of the nuclear charge. The rate coefficients fof excitation. de-excitation, ionization, recombination, emission, and absorption) which determine the papulation of an ionic charge-state are changed very little by the energy shifts within the fine structure, but the rates depend strongly upon the fiuantum numbers af a given fine-structure level.

The code l.INE 2 assumes that He-like fons have only singly excited states, and that these states have fine structure of the form $\left(1 \mathrm{~s}, \mathrm{nl}, 1,3^{3}\right)$ for 
those principal quantum numbers $n$ up to a preassigned value $n_{2 f s} \leq 4$. For larger $n$ the fine-structure states are lumped into a single $n$-level of the form $(15, n)$ with appropriately averaged rate coefficients. Stmilarly LINEZ lumps all states together by $n$-level in the exclted states of $\mathrm{H}-1 \mathrm{ike}$ and Li-like tons.

In order to see how He-like fine structure affects all the populations. the normal LINEZ with $n_{2 f s}=4$ ( 30 excited fine-structure states) was compared to a LINE $Z$ run in which $n_{2 \mathrm{fs}}=2$ (6 excited fine structure states). The results, plotted in Fig. 23, show that the case $n_{2 f s}=2$ ionizes more with some populations varying by up to $70 x$. (Not shown are results for $n_{2 f s}$ $=3$. which are intermediate between those with $n_{2 f s}=?$ and 4.) These differences arise because the lower- $n$ fine structure levels are not populated statisticaliy: and, in lumping together the fine structure of a lower $n$-level, the averaging process enhances the effective ionization mare than the effective recombination. 
The relative populations of charge-states vary widely from one NLIE code to another. This is surprising because the codes use simllar rate coefficients and (except for average-atom XSN) comparable structure in the excited states. It is additionally surprising because the test problems are "easy" . treating optically thin plasmas most of whose jons have two or fewer bound ejectrons. Populations vary from code to cede by as much as 2-1/2 orders of magnitude. although the more typical disagreements are factors of two.

The closest agreements in charge-state populations are between RATION and YTL and between NEBULA, NRL, and PPL, except, in both cases, for Lf-like ArXVI. But these two sets differ from each other by factors as large as 4 (RATION-YTL ionize more than NEBULA-MRL-PPL), and the typical difference is a factor of 1.5 at $2 \mathrm{keV}$.

The agreement with NRL is unexpected in that NEBULA and PPL are coronal equilibrium codes, valid only in the low density timit where ioniration balance is insensitive to density, while NRL and the other codes inciude the effects of higher density upon excited state populations. The density of the NRL run at $10^{21}$ electrons/ce is perhaps three orders of magnitude nigher than the coronal limit in argon.

The average-atom code XSN agrees poorly with the ion population cades at low densities and quite well in dense plasmas. It agrees in $\bar{l}$ at temperatures hot enough that less than 0.5 average electron is bound.

Ratios of net effective tonization/recombination rates disagree from code to code most strongly for the He-like ions, as much as a factor of 50 but more 
typically by factors less than 5 . In general LINEZ fonizes more than the other codes, and XRASER fonizes less.

The effect on charge-state populations due to setected bits of physics is sumarized in Table 2. Of greatest importance are uncertainties in the electron-collisional rate coefficients and the uppermost principal quantum number $n_{\text {max }}$ allawed to each charge-staie.

\section{Table 2.}

Summary of the effect on populations due to selected changes ill physics. All changes are within the code LINEZ sfrulating the optically thin AR plasma at $5.6 \times 10^{19}$ ions/cc.

Cherge within LINF2

\begin{tabular}{|c|c|c|}
\hline $\begin{array}{l}\text { decrease e-call. excit./ } \\
\text { de-excit. by factor of } 2\end{array}$ & less ionization & 1.9 \\
\hline $\begin{array}{l}\text { decrease e-coll. ioniz.l } \\
\text { recomb. by factor of } 2\end{array}$ & $\begin{array}{l}\text { less ionization } \\
\text { above } 2 \mathrm{keV}\end{array}$ & 1.7 \\
\hline $\begin{array}{l}\text { decrease dielectronic recomb. } \\
\text { to zero }\end{array}$ & $\begin{array}{l}\text { less ioniz. for He-like, } \\
\text { Li-like above } 1.5 \mathrm{keV}\end{array}$ & 1.4 \\
\hline $\begin{array}{l}\text { decrease ion-coll. excit./ } \\
\text { de-excit. to zero }\end{array}$ & $\begin{array}{l}\text { more ioniz. for He-like } \\
\text { above } 1.5 \mathrm{keV}\end{array}$ & 1.1 \\
\hline \multirow{2}{*}{ decrease $n_{\max },\left\{\begin{array}{l}16 \text { to } 5 \\
12 \text { to }\end{array}\right.$} & less iontz. & 1.1 \\
\hline & Tess ioniz. & 2.1 \\
\hline $\begin{array}{l}\text { decrease highest } n \text { with } \\
\text { f ine structure, } 4 \text { to } 2\end{array}$ & more ioniz. & 1.6 \\
\hline
\end{tabular}

Typical factor by

Effect on overall ionization balance which significant populations rhange 
The populations computed by various codes seem most at risk from nax being set too small. This is because rate coeffictents are usually computed with care (especially for H-like and He-like jons), while n max may be set arbitrarily low to save computer time. On the other hand most codes do not compute $n_{\text {max }}$ but fix it a priori.

One may reasonably worry that diagnosing a plasma depends no more upon the recorded spectrum than upon which code interpreted it. 
APPENDIX

\section{Continuum Lowering in Plasmas}

Quantum states of partialiy ionized atoms in plasmas are influenced by nelghboring electrons and ions. ${ }^{16}$ These perturbations are relatively minor for states with low princlpal quantum number $n$, as evidenced by measured plasma polarization shifts. In contrast, large perturbations are expected for high-lying states, $n \gg 1$, for which the orbital radius $r_{n}=\langle n|r| n\rangle$ is not small relative to the mean inter-ion spacing $R$. Indeed, when $r_{n} \geq R a$ "bound" electron should be free to move from ion to ion. This situation is referred to as continum lowering or pressure ianization.

One important aspect of continum lowering is not discussed here--those changes in various thermodynamic quantities which are due to modification of atomic partition functions (but see, e.g., 2 immerman and More ${ }^{17}$,. This appendix focuses instead on anly two specific questions:

(1) What is the principal quantum number $\tilde{n}$ of the uppermost bound state of an ion in a plasma?

(2) How many states $n \leq n_{\max } \leq \tilde{n}$ must be treated for accurate calculations of ionization balance in non-LTE, steady-state plasmas?

Our dizcussion assumes that there is a single specie of highly charged ions $(Z>1)$ in the plasma, and that electrons and ions have the same temperature $T$. Generalizations to more complicated situations are straight forward.

One way to obtain a specific answer to the first question is to determine the effective, screened Coulomb potential $V(r)$ binding an electron; this potential results from the ionic core plus the (non-uniform) plasma that has 
been polarized by the ion. Values of $\tilde{n}$ for hot, low-density plasmas have been determined most frequently from the Debye-Hückel model.

$$
\begin{gathered}
V(r)=\frac{+Z \mathrm{e}}{r} \exp \left(\frac{-r}{0}\right), \\
D=\left[\frac{k T}{4 \pi \mathrm{e}^{2} Z \mathrm{~N}_{\mathrm{e}}}\right]^{1 / 2}
\end{gathered}
$$

for which extensive efgenvelue computations exist. ${ }^{18}$ for s-states $(l=0)$. these numerical results are accurately represented by the semi-elassi=al (Bohr-somerfeld) expression 19.20

$$
\tilde{n}_{D H}=\frac{1}{r} \int_{0}^{\infty} d r\left(\frac{2 b(r)}{e a_{D}}\right)^{1 / 2}=\left(\frac{4 Z D}{\pi a_{0}}\right)^{1 / 2},
$$

as long as $\tilde{n}_{D H} \geq 3$. Almost the same formula for $\tilde{n}$ results from the expression for the constant. first-order shift of all hydrogenic eigenvalues when the Coulomb potential is replaced by the Debye-Hückel potential, viz, the continuum lowers by an amount

$$
n_{\mathrm{OH}}=\frac{Z e^{2}}{D}=\frac{z^{2}}{n_{\mathrm{DH}}^{2}} \frac{e^{2}}{2 \mathrm{a}_{\mathrm{O}}}
$$

The Debye-Hückel model is physically meaningful only when the plasma parameter

$$
r=\frac{2^{2} e^{2}}{R k T} \ll l
$$

When the other limit $r \gg 1$ arises, plasma screening of the Coulamb interaction can be approximately represented by the ion-sphere model.

$$
\begin{aligned}
& V(r)=\frac{+2 e^{2}}{r}\left[1-\frac{r}{2 R}\left(3-\frac{r^{2}}{R^{2}}\right)\right], \\
& \frac{4}{3} \pi R^{3} M_{e}=Z .
\end{aligned}
$$


In this model the constant, first-order shift of all hydrogenlc levels gives

$$
\Lambda_{I S}=\frac{3}{2} \frac{2 e^{2}}{R}=\frac{2^{2}}{\tilde{n}_{I S}^{2}} \frac{e^{2}}{2 a_{0}}
$$

which leads to the expression

$$
\tilde{n}_{I S}=\left(\frac{2 R}{3 a_{0}}\right)^{1 / 2}
$$

for the uppermost bound state. The approximate value of $\bar{n}$ obtained from Eo. (A.9) is almost the same as the value we compute, using the semi-classical formula (A.3), for s-states in the fon-sphere potential,

$$
\tilde{n}=\frac{3}{2 \pi} \ln (2+\sqrt{3})\left(\frac{Z R}{a_{0}}\right)^{1 / 2}=1.09\left(\frac{Z R}{3 a_{0}}\right)^{1 / 2}
$$

Stewart and Pyatt ${ }^{21}$ derived a simple analytic expression that often is used to compute continum lowering in the intermediate regime $r \sim 1$,

$$
n_{S P}=\frac{k T}{2 Z}\left\{\left[(3 r)^{3 / 2}+1\right]^{2 / 3}-1\right\} .
$$

This formula reduces to $\Lambda_{I S}$ and $\Lambda_{D H}$ in the $h i g h-$ and low-r limits, respectively. since $\sqrt{3 \Gamma}=R / O$, and it gives as the uppermost bound state

$$
\tilde{n}_{S P}=\tilde{n}_{I S}\left\{\frac{3 \Gamma}{\left[(3 \Gamma)^{3 / 2}+\right]^{2 / 3}-1}\right\} \text {. }
$$

There exist more sophisticated descriptions of static screening effects in a plasma, such as the Hartree-Fock-Dirac average atom ${ }^{22}$ and finite-temperature density functional ${ }^{23}$ models. Unfortunately. because these schemes do not yield expressions for $V\langle r\rangle$ in closed form, they are not suitable for simple estimates of $\tilde{n}$. Therefore, even though the Stewart-Pyatt model does have sonue 
shortcomings at large $r$-values (for example, it overestimates the particle energy density by about $15 \%$, and it is not applicable to degenerate plasmas ${ }^{24}$, the continuum lowering formulae of Eqs. (A.11) and (A.:2) should be adequate for computations related to plasma spectroscopy.

It is easy to show that the ion-sphere value of $\tilde{n}$ also results from a consideration of the static Stark effect due to plasma ions. Altogether. these ions praduce a microfield of characteristic strength

$$
\langle\mathrm{F}\rangle=\frac{3 \mathrm{Ze}}{\mathrm{R}^{2}}
$$

Approximate hydrogentc eigenvalues in the presence of this fieid are

$$
E_{n} \simeq\left[\frac{-z^{2}}{n^{2}}+\frac{3<F>n^{2}}{2}\right] \frac{e^{2}}{2 a_{0}} \text {, }
$$

when $\langle F\rangle$ is measured in atomic units $\left(e / a_{0}^{2}\right)$. The uppermost level is gotten from setting $E_{n}=0$, whence

$$
\tilde{n}_{\text {Stark }}=\left(\frac{2 R}{3 a_{0}}\right)^{1 / 2}=\tilde{n}_{\text {IS }} \text {. }
$$

Yet another scheme for estinating $\tilde{n}$ was suggested to us by $\Lambda$. 5zöke ${ }^{25}$; The density of bound electrons in an excited level $n$ should not exceed the density of free electrons in the small energy interval $\left(0 \leq E \leq \varepsilon_{n}\right)$. where $c_{n}$ corresponds to the "width" of the bound level. Because levels near the continuum are almost in equilibrium (LTE) with it, we can use this restriction in conjunction with the Saha-Boltzmann equation to write

$$
N \simeq N_{n}^{L T E} \leq N e \int_{0}^{\epsilon n} f(E) d E
$$


with $f(E)$ being the distribution function for electron energies. If both the width and the binding energy of level $n$ are much less than $k T$, and if $f(E) i s$ Maxwelltan, then Eq. (A.16) reduces to

$$
n^{2} \leq \frac{z}{6 r^{2} a_{0}^{3} \omega}\left(\frac{c n}{e^{2} / 2 a_{0}}\right)^{3 / 2}
$$

By taking the level width to be equal to the level spacing, in $=$ $z^{2} e^{2} / n^{3} a_{0}$. we obtain an uppermost level that is nearly the same as the fon-sphere value,

$$
\tilde{n}_{\text {Szöke }}=\left(\frac{28}{1.7 a_{0}}\right)^{6 / 13}=1.3 \tilde{n}_{I S}^{12 / 13} .
$$

It is interesting to compare these various results with the uppermost spectroscopically discernable level $\tilde{n}_{1 T}$, as given by the Inglis-Teller formula, ${ }^{26}$ which matches the level spacing and the linear stark width. This gives, for $\tilde{n} \gg 1$.

$$
\frac{z^{2}}{\pi^{3}} \frac{e^{2}}{a_{0}}=\frac{3<F>\tilde{n}^{2}+a_{0}}{2}
$$

or

$$
\tilde{n}_{I T}=\tilde{n}_{I S}^{4 / 5}=0.73 \Gamma^{1 / 5} \tilde{n}_{D H}^{4 / 5} .
$$

Roussel and 0 'Conne $11^{27}$ considered the situation where $\tilde{n}_{D H}<\tilde{n}_{I T}$. According to Eq. (A.20), this is tantamount to $\tilde{n}_{D H}<r / 4.9$. But. the Debye-Hückel picture is only valid when $r<1$, so their discussion actually applies only to the irrelevant case of no bound states.

How many of the extant bound states are relevant to the calculation of ionization balance in a particular non-cTE plasma could be determined by 
successive computations, with $n_{\max }=\tilde{n}, \tilde{n}-1, \ldots$ etc., which continue until unacceptable erros result. However, one would like to have this knowledge a priori. so that the smallest possfble atomic data base need be assembled and the least possible amount of computer time need be used.

This issue was addressed in the work of Bates et al... 11 who were the first to compute dense plasma lonization and recombination rate coefficients with their collisional-radiative model. They argued that an electron in an excited level $n$ should be considered bound only if it could complete at least one 8ohr orbit in a time $(a \simeq 1 / 137)$

$$
\operatorname{torb}_{\text {or }}(n)=\frac{2 \pi n^{3} a_{0}}{z^{2} a c}
$$

that is short compared with its lifetime against radiative or collisional destruction

$$
T_{1 i f e}(n)=\left[\tau^{-1} \operatorname{rad}^{(n)}+\tau_{\operatorname{col1}}^{-1}(n)\right]^{-1} ;
$$

otherwise, a transition involving an ion in such a state would not be a distinct event.

An approximate analytical expression of this inequality may be obtained as follows. First, we assume that the plasma density is high, so that for the uppermost bound states $\tau_{\text {coll }} \ll \tau_{\text {rad }}$ Then,

$$
{ }^{\tau} 1 \text { ife } e^{(n)}=\left[N_{e}<\theta_{i n}(n) v>\right]^{-1} \text {. }
$$

where $Q_{1 n}(n)$ is the total inelastic cross section for transitions out of level $n$ and $\langle\ldots$.$\rangle represents a thermal averaging. Next, we use the$ Weisskopf radius $\rho_{W}$ to estimate a specific cross section, and we include a logarithmic factor to give the correct high-energy behavior, ${ }^{2 B}$ 


$$
a(a, b)=\pi \rho_{w}^{2} \ln \left(\frac{4 E}{\Delta E}-1\right)=\frac{\pi^{3} e^{2}}{2 a a^{E}}(a|r| b)^{2} \ln \left(\frac{4 E}{\Delta E}\right) \text {. }
$$

where $(a|r| b)$ is the (dipole) transition moment and $\Delta E$ is the transition energy. The logarithmic term is needed because $\Delta E / E \ll 1$ for Rydberg levels. To simplify the final expression for $Q_{i n}(n)$. We now assume that all scattering channels are open (i.e., they represent de-excitations or, if excltations. transitions with $\Delta E \ll k T$ ) and we replace $\Delta E$ in the logarithmic term with the energy for the $\Delta n=1$ transition, $\Delta E+2 Z^{2} I_{H} / n^{3}$. Thereafter, we use the completeness property of the atomic states to obtain

$$
\begin{aligned}
Q_{i n}(n) & =\sum_{b \neq a} Q_{i n}(a, b)=\frac{\pi^{3} e^{2}}{2 a_{0} E} \ln \left(\frac{2 n^{3} E}{z^{2} I_{H}}\right) \sum_{b \neq a}(a|r| b)^{2} \\
& =\frac{\pi^{3} e^{2}}{2 a_{0} E} \ln \left(\frac{2 n^{3} E}{z^{2} I_{H}}\right)\left[\left(a\left|r^{2}\right| a\right)-(a|r| a)^{2}\right] \\
& =\frac{\pi^{3} e^{2}}{2 a_{0} E} \ln \left(\frac{2 n^{3} E}{z^{2} I_{H}}\right)\left[\frac{2 n^{4}+8 n^{2}-1}{12 z^{2}}\right] .
\end{aligned}
$$

where. In the last 1 ine of $\mathrm{Eq}$. (A.25), we have averaged with respect to orbital quantum numbers Finally, we approximate the Maxwellian average by

$$
\begin{aligned}
\left(Q_{i n}(n) v\right) & =\frac{\pi^{3} e^{2} a_{0} n^{4}}{12 z^{2}} \ln \left(\frac{3 n^{3} k T}{z^{2} I_{H}}\right)\left\langle\frac{v}{L}\right\rangle \\
& =\frac{\tau^{5 / 2} a a_{o}^{2} c}{3} \frac{n^{4}}{z^{3}}\left(\frac{z^{2} I_{H}}{k T}\right)^{1 / 2} \ln \left(\frac{3 n^{3} k T}{z^{2} I_{H}}\right) .
\end{aligned}
$$


Thus, the uppermost level according to the criterion of Bates et al. ${ }^{11}$ is given by

$$
\begin{aligned}
{\left[n_{\max }(\theta K M)\right]^{7} } & =\left(\frac{54 Z}{5 / 2}\right)\left(\frac{Z R}{3 a_{0}}\right)^{3}\left(\frac{k T}{z^{2} I_{H}}\right)^{1 / 2} / \ln \left(\frac{3 k T n_{\max }^{3}}{z^{2} I_{H}}\right) \\
& =3.12 \tilde{n}_{I S}^{6}\left(\frac{k T}{z^{2} I_{H}}\right)^{1 / 2} / \ln \left(\frac{3 k T n_{\max }^{3}}{Z^{2} I_{H}}\right) .
\end{aligned}
$$

Since, typically. $k T \sim 1 / 4$ to $1 / 3$ times $z^{2} I_{H}$, we have simply

$$
n_{\max }^{7}(B \times M) \text { en }\left[n_{\max }(B K M)\right]=\frac{1}{2} I \tilde{n}_{15}^{6} .
$$

For values of $n_{\max }$ between. say, 5 and $50,2 n n_{\max }-3$ and evidently then $n_{\max }\left(\right.$ (BKM) is not substantially less than $n_{I S}$.

Is there a more restrictive criterion one can apply? Yes; it is clear from the steady-state population equations that an excited level in strict LTE with the overlying continum does not contribute to net rates of jonization or recombination. Only those levels for which $\tau_{\text {rad }}^{-1}$ is a significant fraction of ${ }^{-1}$ coll are out of LTE with the continuum, so we can estimate n max from the critericn

$$
\begin{aligned}
{ }^{\top} \operatorname{coll}\left(n_{\max }\right) & \geq(1 / \xi) \tau_{\operatorname{rad}}\left(n_{\max }\right) \\
& =2.6 \times 10^{6} \frac{n^{9 / 2} a_{0}}{\xi Z^{4} \text { or }} .
\end{aligned}
$$

where the number $\xi \gg 1$, and where we have used the asymptotic nydrogenic value for the excited-state radiative lifetime. since $t$ rad is always much greater than sorb for any level, formula (A.29) can be more restrictive than the Bate . al. ${ }^{11}$ criterion for $n_{\max }$. If we use Eq. (A.26) to determine 
Toll, and adopt the typical values $k T \sim 1 / 4$ to $1 / 3$ times ${ }^{2}{ }^{2}{ }_{H}$ and $\ln \left(n_{\max }\right) \sim 3$, the LTE departure criterion yeilds an uppermost lavel

$$
n_{\max }(N L T E)=\left[\xi\left(Z \alpha \tilde{n}_{I S}^{2}\right)^{3}\right]^{2 / 17} .
$$

Our experience in this and other investigations suggests that, in dense plasmas. level with LTE departures of older $0.1 \%$ or more can measurably contribute to ionization/recombination rate coefficients. Consequently, for simple estimates of $n_{\max }$ we recommend the value $\xi=10^{3}$ be used in Eq. (A. 30).

It is possible to obtain another estimate of $n_{\max }$ by considering the effects of plasma lans. However, instead of inducing transitions via collisions, the slower moving ions are treated as the source of a microfield which, if it fluctuates to a high value $F \gg(F)$, may ionize levels below $\tilde{n}_{\text {Stark }}=\tilde{n}_{15}$. For this to happen, the strong field must (1) exist long enough that a bound electron can respond to $i t$, and (2) arise on a timescale short enough to compete with radiative decay. If $\nu\left(F_{n}\right)$ is the frequency of occurence of fields strong enough to ionize a level $n$, and $\tau\left(f_{n}\right)$ is the characteristic lifetime of said field, these two requirements can be expressed as

$$
\begin{aligned}
& \tau\left(F_{n}\right)>\tau_{\operatorname{arb}}(n) . \\
& v\left(F_{n}\right)>1 \tau_{\operatorname{rad}}(n) .
\end{aligned}
$$

In order to obtain a simple formula for $v\left(F_{n}\right)$, we assume that the micrafield flirtuations are a Poisson step process, with a hopping frequency independent of field strength and equal to the fon plasma frequency, 


$$
v=\frac{1}{2 \pi} \omega_{2}=\frac{1}{2 \pi}\left(\frac{4 \pi Z e^{2} N_{e}}{H_{2}}\right)^{1 / 2}
$$

Then, $v\left(F_{n}\right)$ is just $u$ times the probability $P\left(\geq F_{n}\right)$ that the instantaneous field strength is at least as large as $F_{n}$. For fields $F>(F)$, the Holtsmark distribution yieids

$$
\begin{aligned}
P\left(\geq F_{n}\right) & =\left(\frac{\langle F\rangle}{3 F_{n}}\right)^{3 / 2} \\
& =\left(\frac{1}{3}\right)^{3 / 2}\left(\frac{n}{\tilde{n}_{1 S}}\right)^{6} .
\end{aligned}
$$

where $(F)$ is the mean field defined in Eq. (A.13). Upon using Eqs. (A.33), (A.32), and (A.29), the inequality (A.3)a) yielos the lowest level for which Lorentz ionization can compete with radiative decay:

$$
n \geq n_{<}(F)=\tilde{n}_{1 S}\left[0.54\left(Z / \tilde{n}_{I S}\right)^{1 / 7}\left(M_{2} / M_{H}\right)^{1 / 21}\right]
$$

If the bracketed quantity is less than unity, one then determines, via Eq. (A.3la) for which (if any) levels $n$ in the range $n_{<}(F) \leq n \leq \tilde{n}_{\text {Is }}$ the strong fields are of sufficient duration to effect iontzation.

According to Chandrasekhar and von Neumann ${ }^{2 g}$ strong $f$ ields $F \gg(F)$ have mean lifetimes

$$
r(F)=\frac{0.15}{N_{Z}{ }^{1 / 3}}\left(\frac{M_{2}}{k T}\right)^{1 / 2}\left(\frac{\langle F)}{F}\right)^{1 / 2},
$$

from which it follows that (for $n<\tilde{n}_{\text {IS }}$ )

$$
T\left(F_{n}\right)=\frac{1 . o_{n}^{2}}{z^{2}}\left(\frac{z^{2} I_{H} H_{z}}{k T m_{e}}\right)^{1 / 2} \frac{a_{0}}{a c} .
$$


This expression, together with Eq. (A.21). yields the uppernost state satisfying the inequality of Eq. (A.3la):

$$
\begin{aligned}
n\left\langle n_{>}(F)\right. & =6 . \theta\left(H_{Z} / H_{H}\right)^{1 / 2}\left(Z^{2} I_{H} / k T\right)^{1 / 2} \\
& =\tilde{n}_{I S}\left[B .4\left(\Gamma H_{Z} / Z H_{H}\right)^{1 / 2}\right] .
\end{aligned}
$$

As long as the coulomb paraneter $r \geq 0.008$, the bractieted quantity above will be greater than unity, and all bound levels $n<\tilde{n}_{1 S}$ will satisfy the restriction (A.3la). (It should be mentioned that no levels satisfy this restriction vis-a-vis electron-microfield fluctuations.) Thus, when $n_{>}(f)$ exceeds $\tilde{n}_{I S}$ and $n_{<}(F)$ is less than $n_{>}(F)$ we can identify

$$
n_{\max }(F)=n_{<}(F)
$$

as the uppermast bound level, higher levels being efficiently Lorentz ionized. As a final item, we compare $n_{\max }$ (NLTE) to $n_{\max }(F)$. Their ratio is ( $\xi$ $=10^{3}$ )

$$
\begin{aligned}
\frac{n_{\max }(N L T E)}{n_{\max }(F)} & =0.73\left(\frac{M_{H}}{M_{Z}}\right)^{0.048}\left(\frac{z^{0.21}}{\tilde{n}_{\text {IS }}^{0.15}}\right) \\
& =0.7\left(\frac{Z}{\tilde{n}_{\text {IS }}}\right)^{0.15} .
\end{aligned}
$$

with the second line following from the fact that $M_{Z} \leq 22 M_{H}$. We conclude that these two prescriptions yield very similar values of $n_{\max }$. 


\section{REFERENCES}

1. Y. T. Lee, "Ionization Balance in Non-equilibrium Plasmas." Lawrence Livermore National Laboratory internal report UCID-18574-83-3 (1983); Y. T. Lee, "An Ionization Balance Model for Non-equilibrium Plasmas," to be published in 3 . Quant. Spectrosc. Radiat. Transfer.

2. W. A. Lokke and W. H. Grasberger, "XSNQ-U: A Non-LTE Emission and Absorption Coefficient Subroutine," Lawrence Livermore National Laboratory report UCRL -52276 (1977).

3. P. L. Hagelstein, "Physics of Short Wavelength Laser Design," (Ph.D. thes is) Lawrence Livermore National Laboratory report UCRL-531C0 (Jan. 1981):

P. L. Hagelstein, S. Morgan, R. L. Huff, L. Minner, "XRASERdOC," instruction manual avaflable fron L. Minner, Lawrence Livermore National Laboratory, L-16, P.0. Box B0B, Livermore, CA 94550 (Nov. 1983).

4. A. W. Lee. B. L. Whitten, and R. E. Strout I [, J. Quant. Spectrose. Radiat. Transfer 32, 91 (1984).

5. R. A. Hulse, Nuclear Technology/Fusion 3, 259 (1983),

6. D. Duston, J. E. Rogerson, J. Davis, and M. Blaha, Phys. Rev. $A$ 28, 2968 (1983). 0. Quston, "Detailed Modeling of Ionization/Radiation in Dense Plasmas," to be published in J. Quant. Spectrosc. Radial. Transfer.

T. C. B. Tarter and E. E. Sa Ipeter. Astrophysical J. 156, 953 (1989); 3. C. Weisheit, 6. A. Shields, and C. B. Tarter, Astrophysical J., 245, $406(1981)$. 
8. J. C. Weisheit, C. B. Tarter, J. H. Scofield, and L. M. Richards, J. Quant. Spectrosc. Radiat. Transfer 16, 659 (1976);

W. E. Alley, G. Chapline, P. Kunasz, and J. C. Weisheit, J. Quant. Spectrose. Radiat. Transfer 27, 257 (1982).

9. T. Fujlmoto and T. Kato, Phys. Rev. A 30, 379 (1984). Their effective rate coefficients are called $S_{C R}$ and ${ }^{a} C R$; these are tabulated in a research report, IPPJ-647 from the Institute of Plasma Physics, Nagoya University. Nagoya, Japan (Sept. 1983).

10. H. H. Grasberger, private communication to R. J. Fortner, LLNL (Dec. 198i).

11. D. R. Bates, A. E. Kingston, and R. W. P. McWhirter, Proc. Royal Soc. London A 267, 297 (1962).

12. J. J. Thomson, Phit. Mag. 23, 419 (1912).

13. W. Lotz, Astrophysical J. Suppl. 14, 207 (1967).

14. A. Jacobs, J. Quant. Spectrosc. Radiat. Transfer 12, 243 (1972).

15. S. M. Younger, J. Quant. Spectrosc. Radiat. Transfer 26, 329 (1981).

16. J. C. Weisheit, "Applied Atomic Collision Physics," Vol. II, ed. C. F. Barnett (Academic Press. New York 1984).

17. G. B. Zimmerman and R. M. More. J. Qucant. Spectrosc. Radiat. Transfer 23, 517 (19B0).

18. F. J. Rogers, H. C. Graboske, Jr., and D. J. Harwood, Phys. Rev. A 1, 1577 (1970).

19. A. S. Dickinson, J. Phys. B4, L116 (1971).

20. K. Chandan, Nuovo Cimento 58A, 191 (1968).

21. J. C. Stewart and K. D. Pyatt, Jr., Astrophys. J. 144, 1203 (1966). 
22. B. F. Rozsnyai, Phys. Rev. A5, 1137 (1972).

23. U. Gupta and A. K. Rajagopal, Phys. Reports 87, 259 (1982).

24. R. M. More. "Atomic Physics in Inertial Confinement Fusion." Lawrence Livermore National Laboratory report, UCRL-84991 (1981).

25. A. Szöke, private communication.

26. D. R. Inglis and E. Teller, Astrophys. .. 90, 439 (1939).

27. K. M. Roussel and R. F. D'Conne11, Phys. Lett. 51A, 244 (1975).

28. R. J. W. Henry. Phys. Reports 68, 1 (1981).

29. S. Chandrasekhar and J. yon Neumann, Astrophys. J. 95, 489 (1942). 
Code:

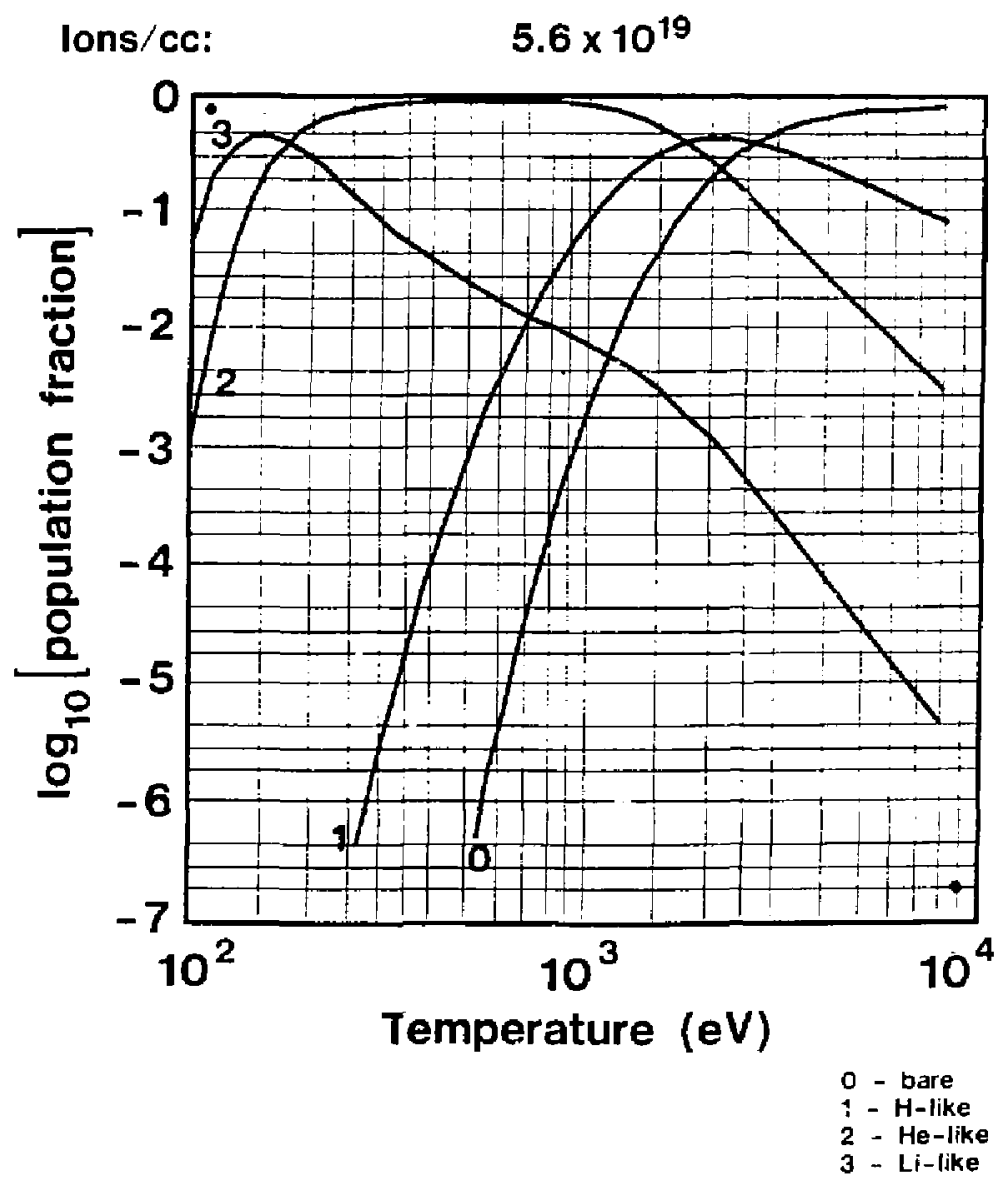

An example of how charge-state populations are plotted. The code YTL computes the population fraction of four ions, those with $0,1,2$, and 3 bound electrons. The plasma is optically thin argon at a density $n_{i}=5.556 \times 10^{19}$ ions/cc, and temperatures vary 'rom 100 to $10,000 \mathrm{eV}$. Not shown are the population fractions of ionic charge-states having more than three bound electrons. 
Code:

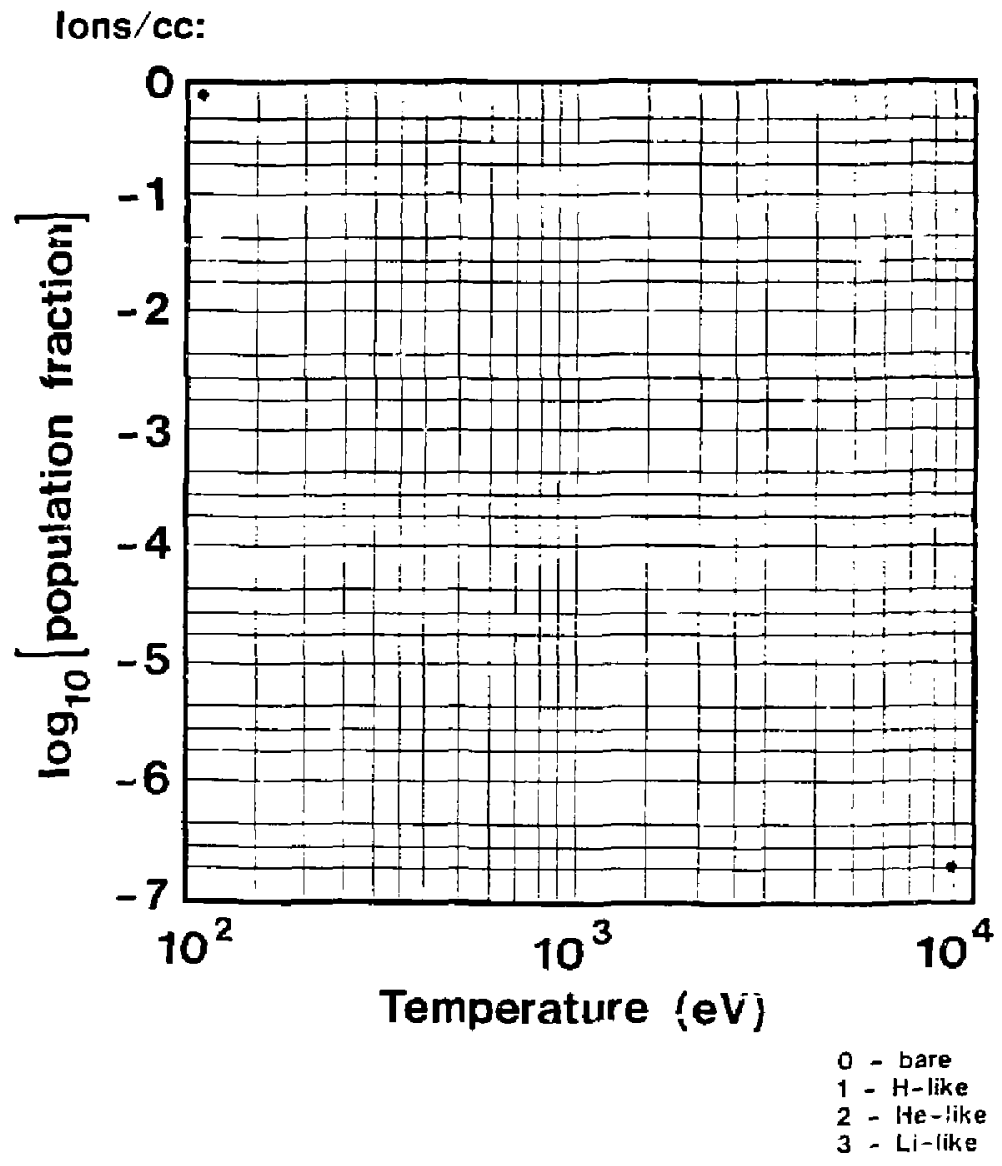

Comparison of charge-state populations. The same $\log -\log$ grid of Fig. 1 calibrates here the curves on six transparent overlays. Each overlay has the C., 1-, 2- and 3-electron ionic populations for an optically thin argon plasma at $n_{i}=5.6 \times 10^{19}$ ion/cc, with a different overlay for each code's computation. The codes are RATION, XRASER, LINEZ, YTL, FOREST, and NRL. Two color dots on each cverlay align with two dots on the $\log -\log$ grid (upper left and lower right corners). 


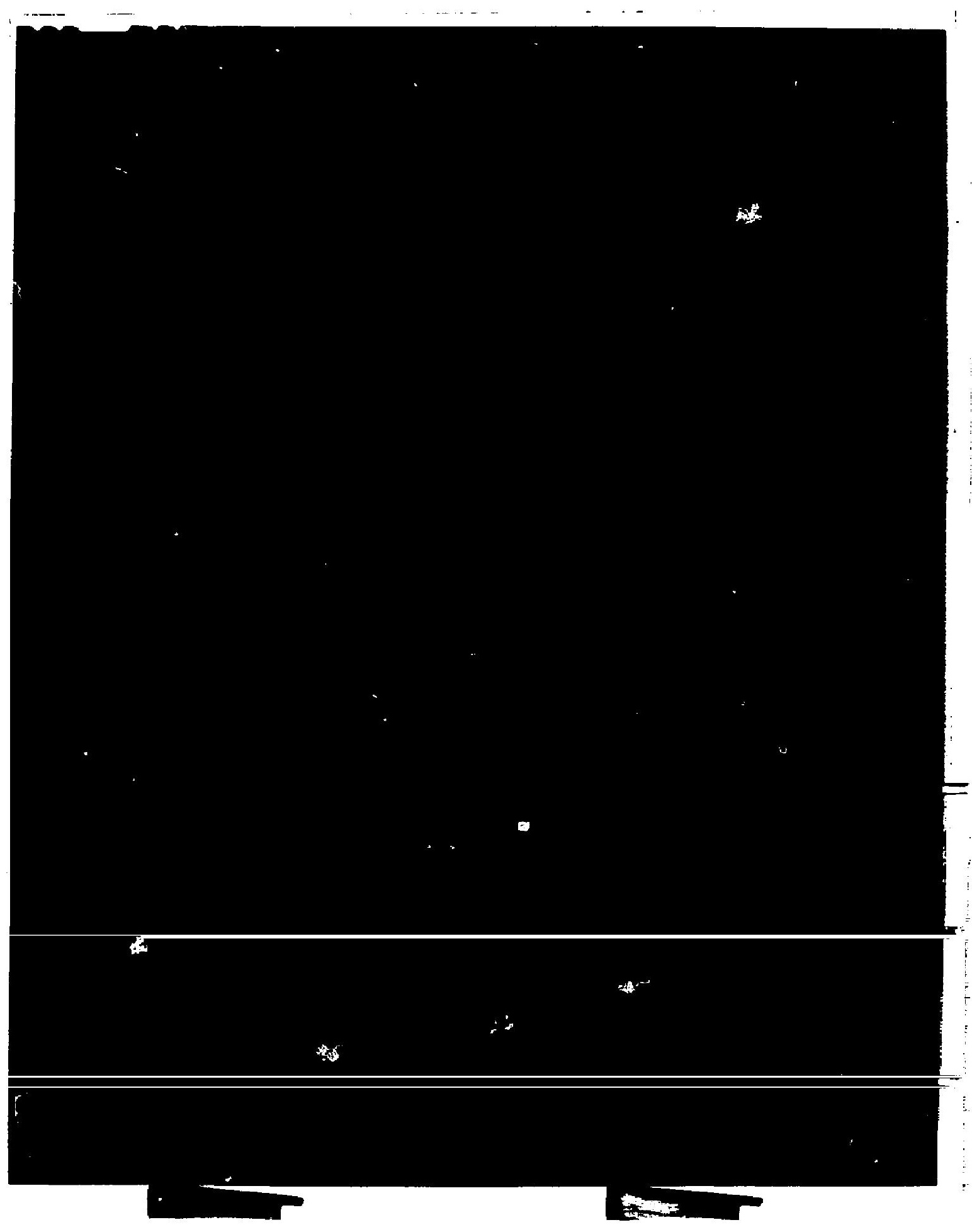





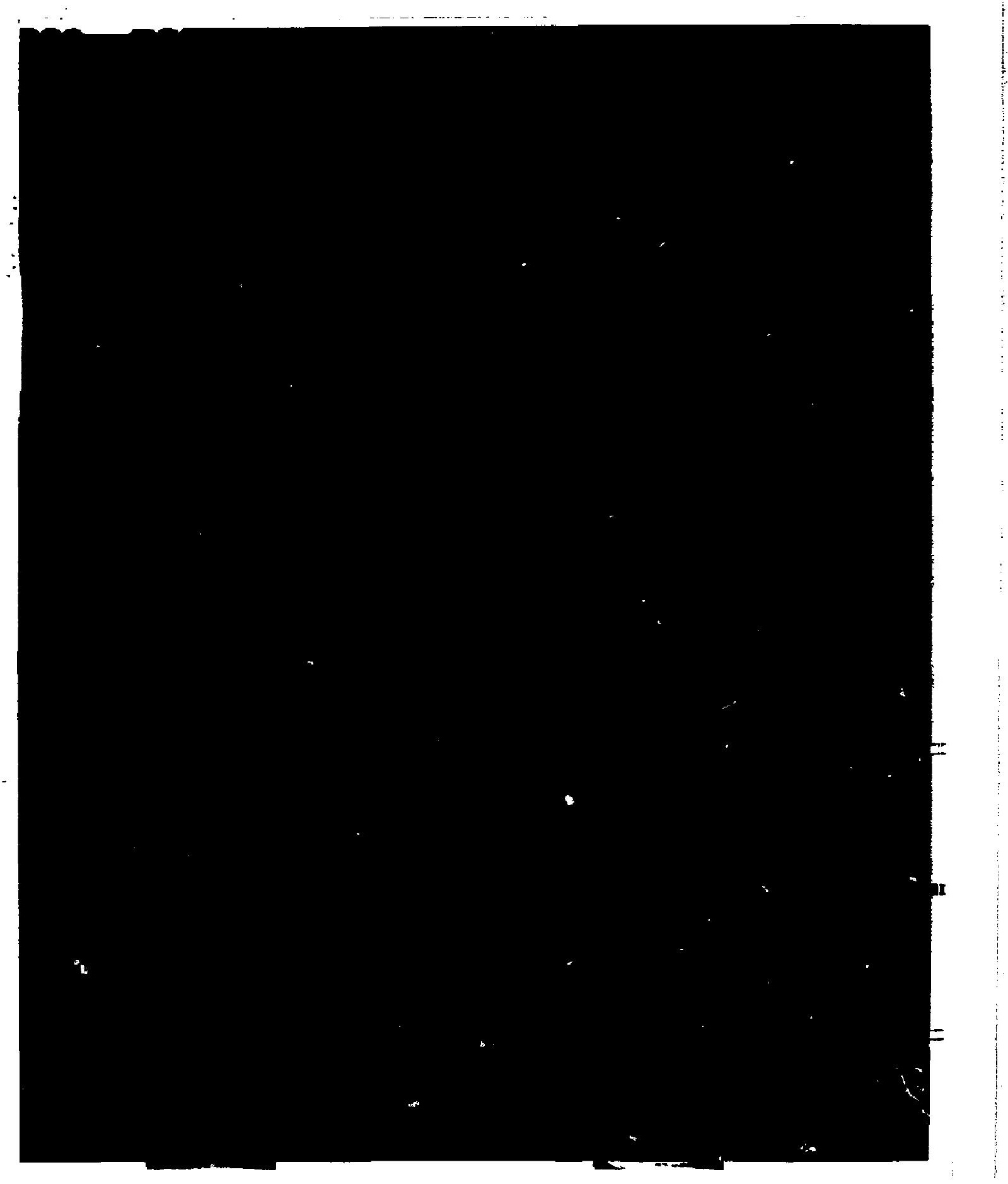




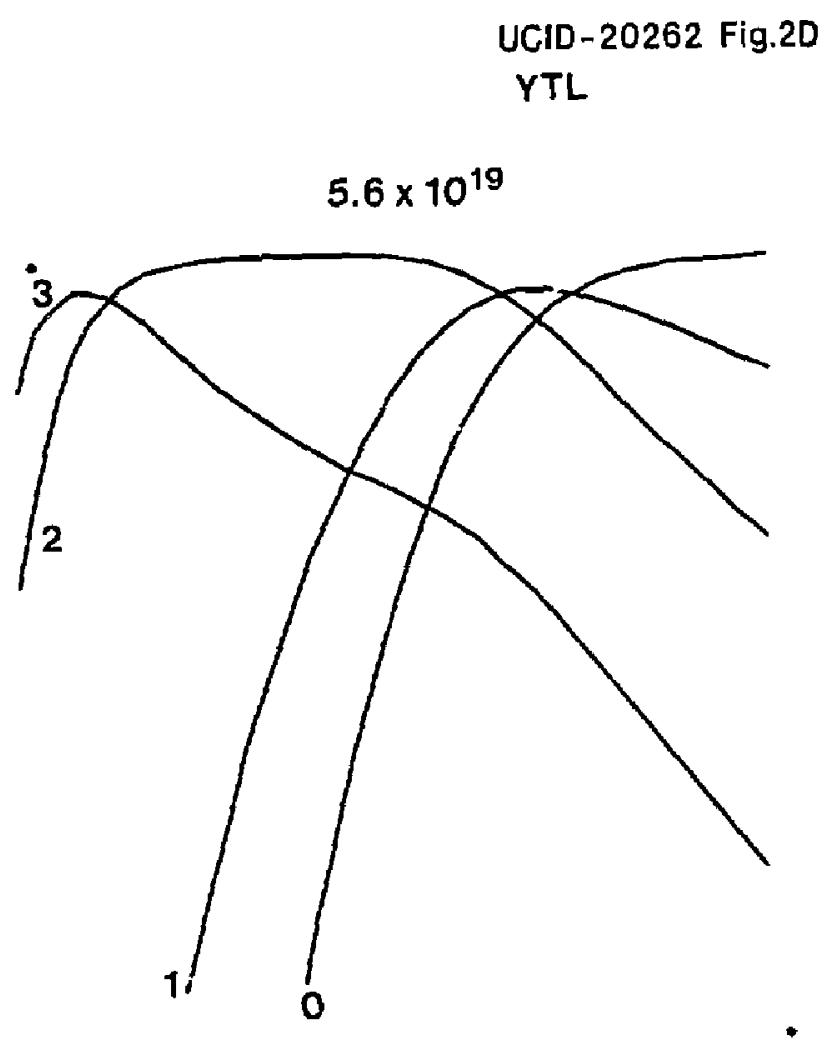




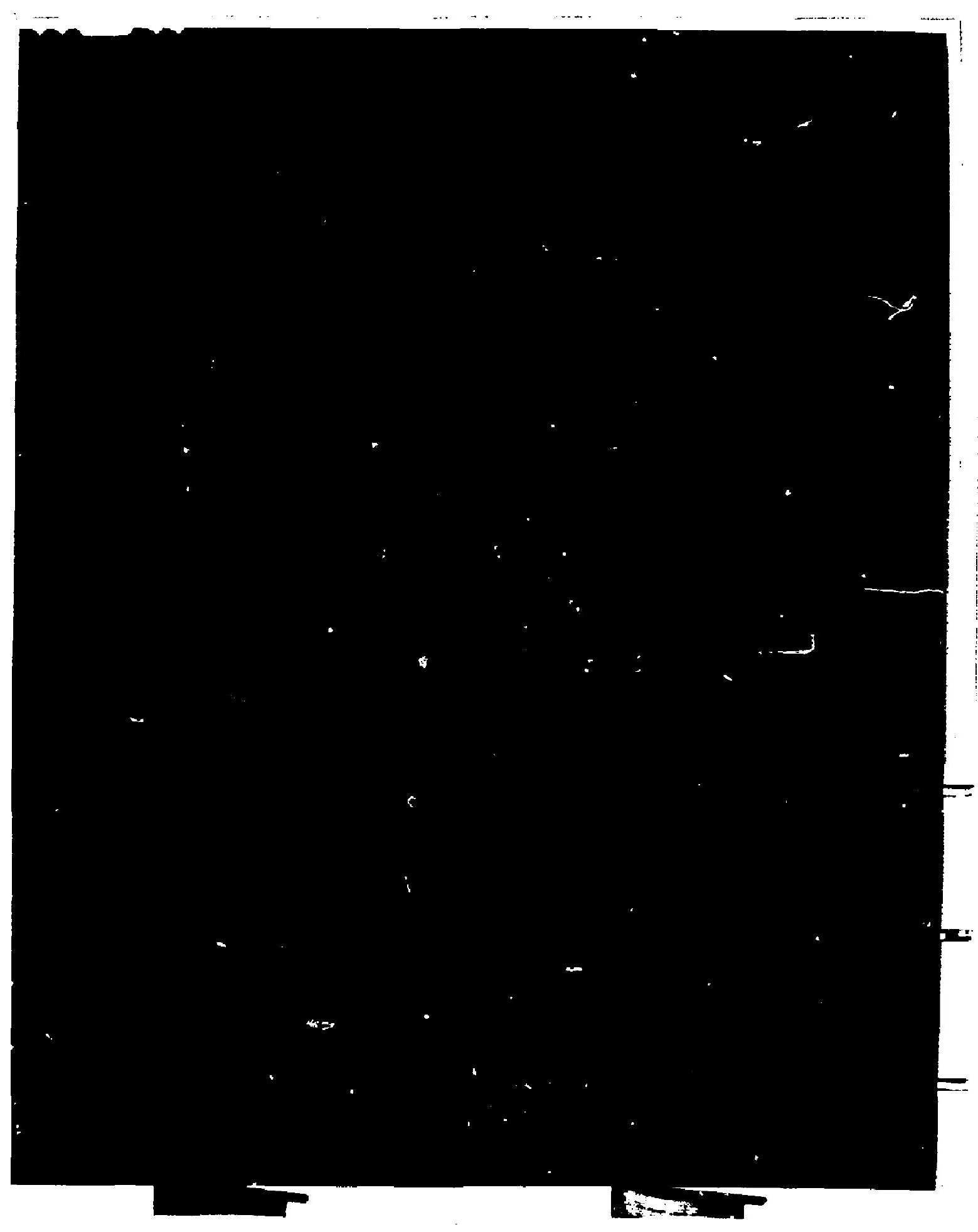




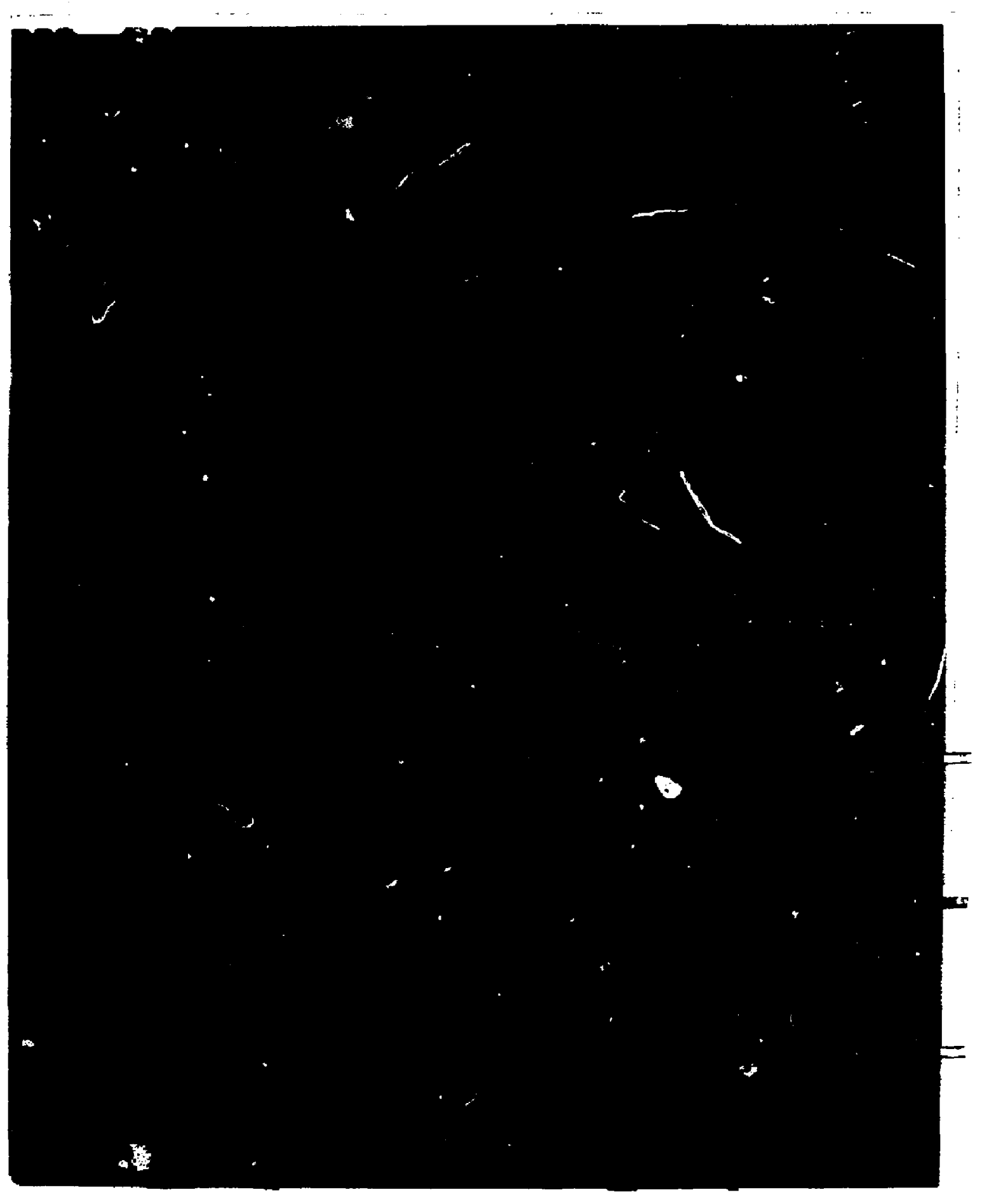


Code:

UCID-20262 Fig.3

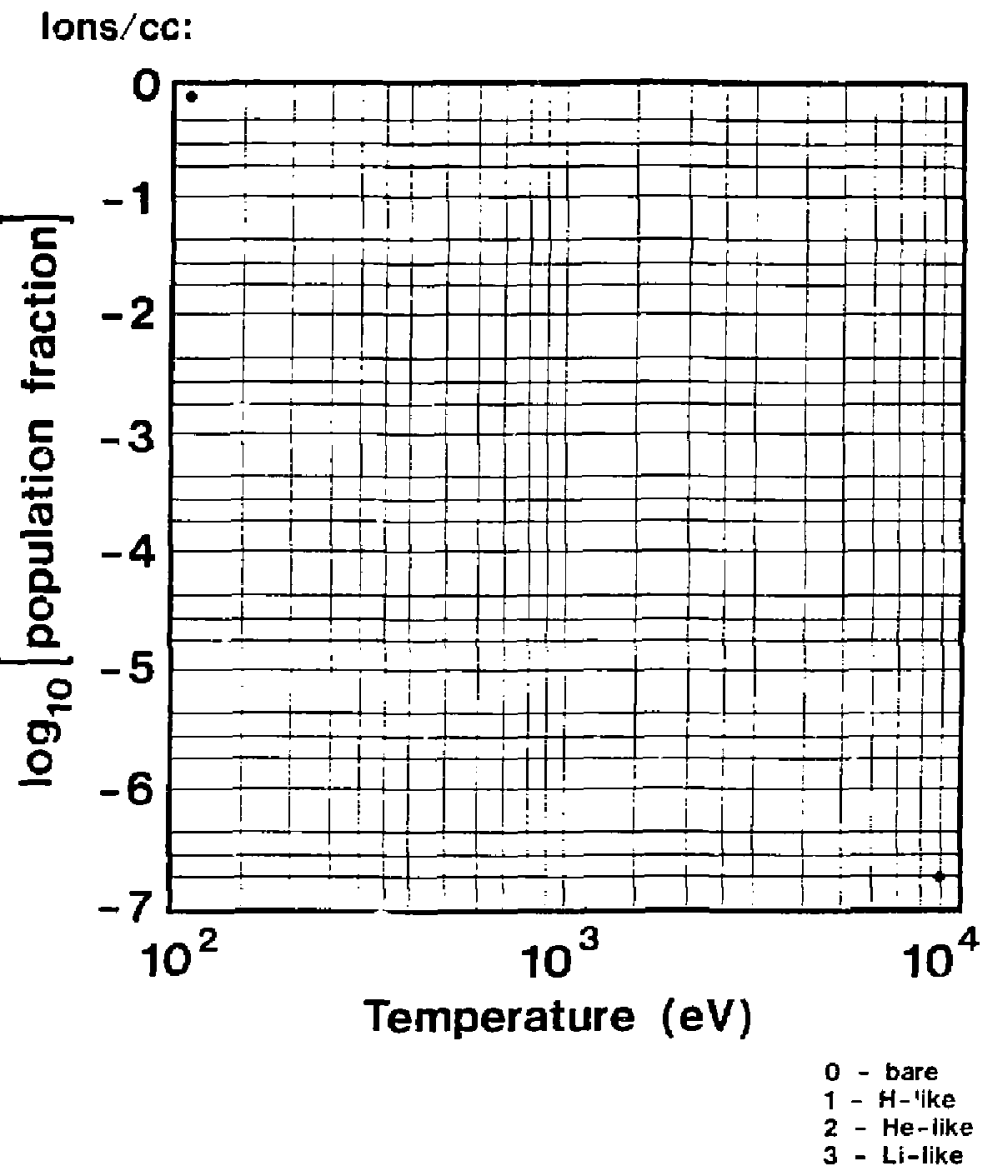

Comparison of charge-state populations. As in Fig. 2, a log-log grid calibrates the curves on five transparent overlays, one for each of five codes. The codes are RATION, XRASER, LINEZ, NEBULA, and PPL. Here the optically thin argon plasma has a density $n_{i}=5.6 \times 10^{17}$ ions/cc. 


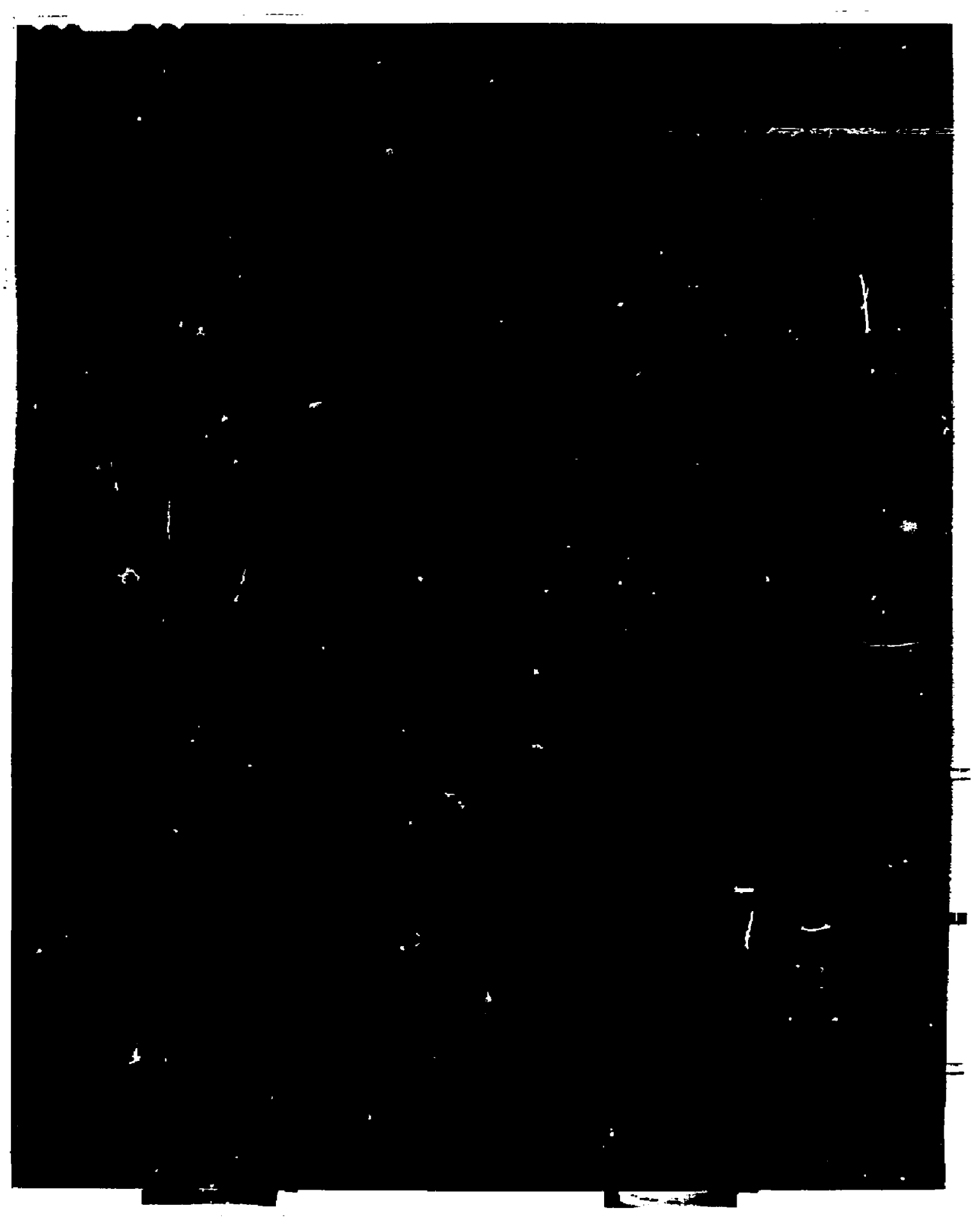




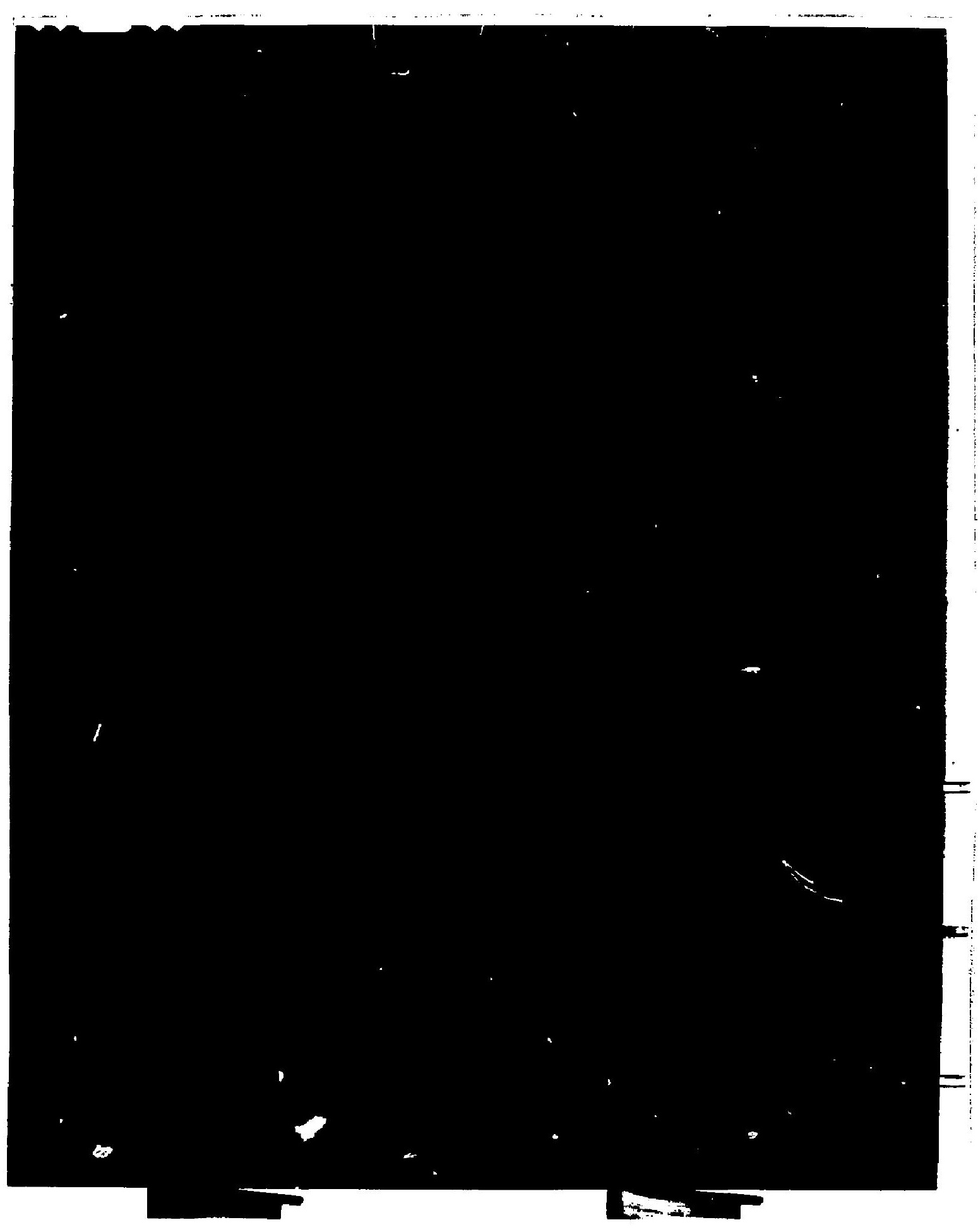




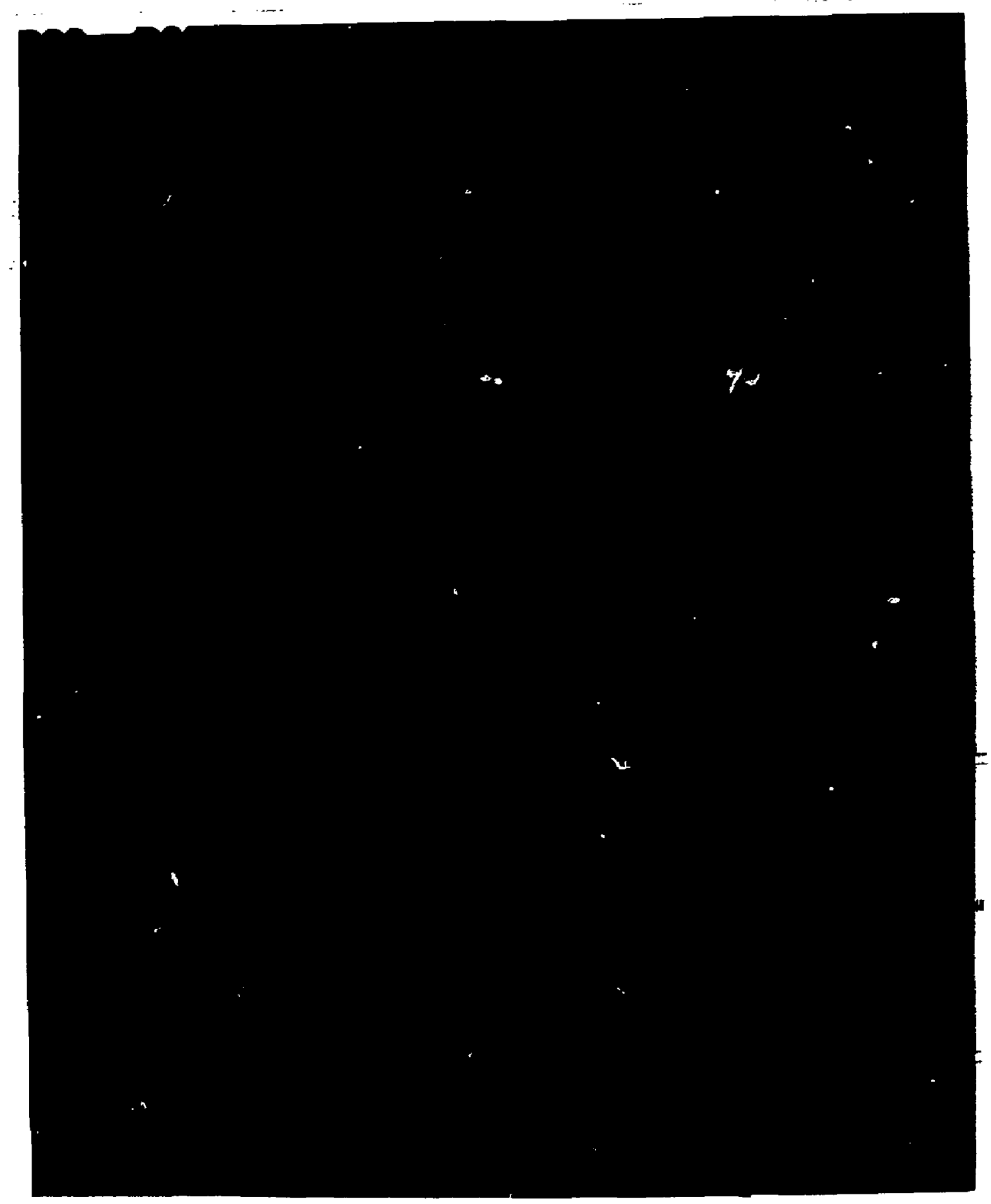




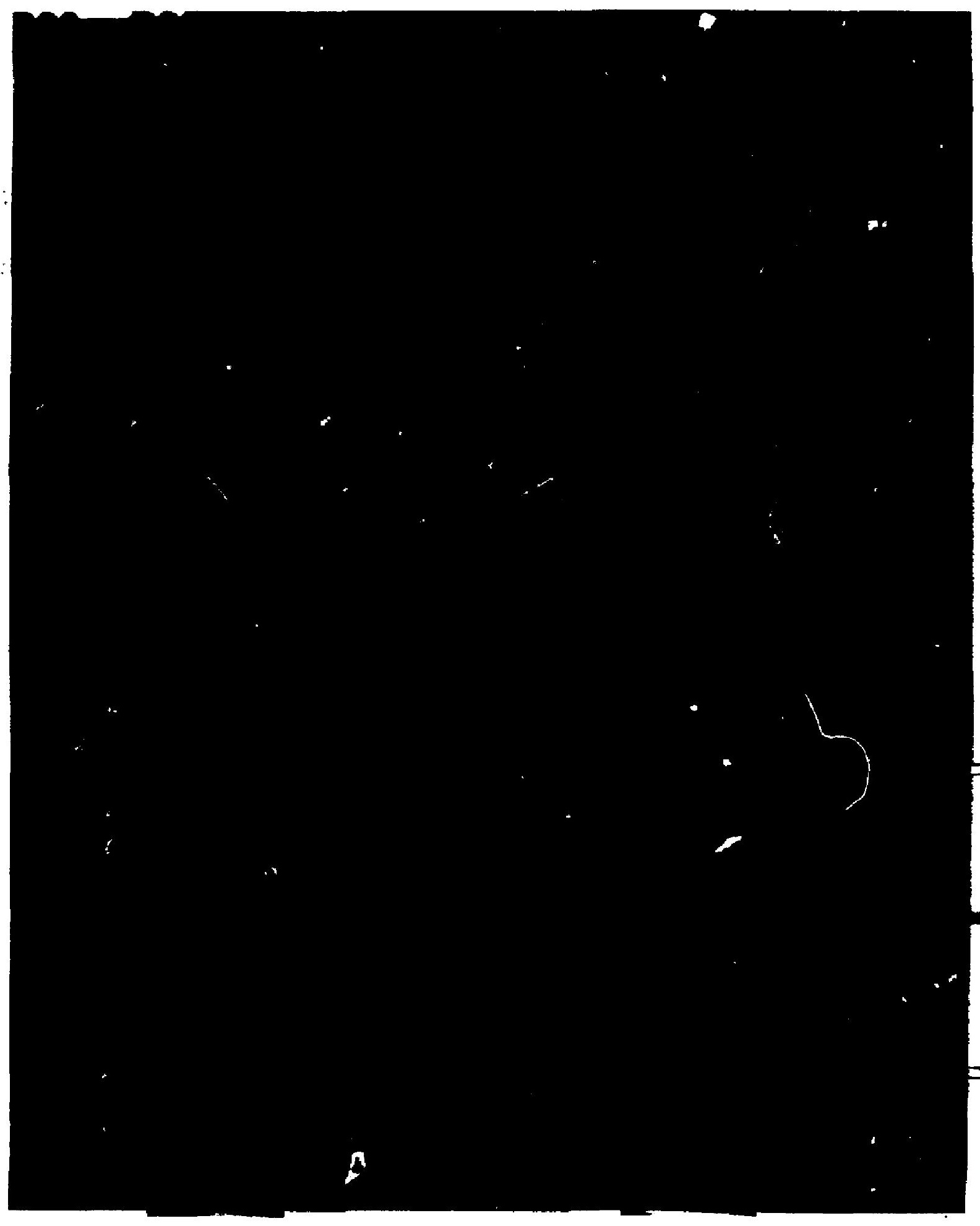




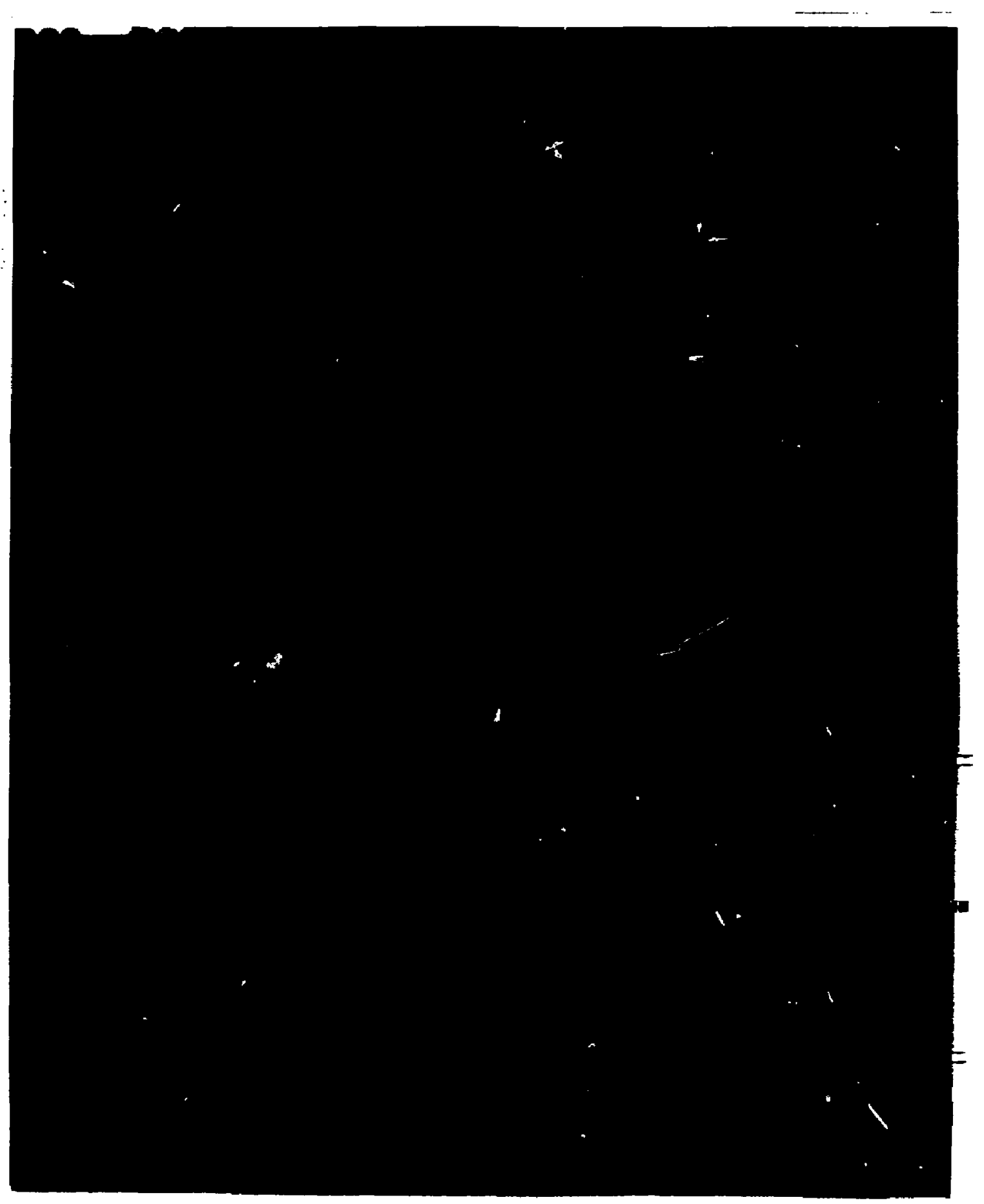


Code:

UCID-20262 Fig.4

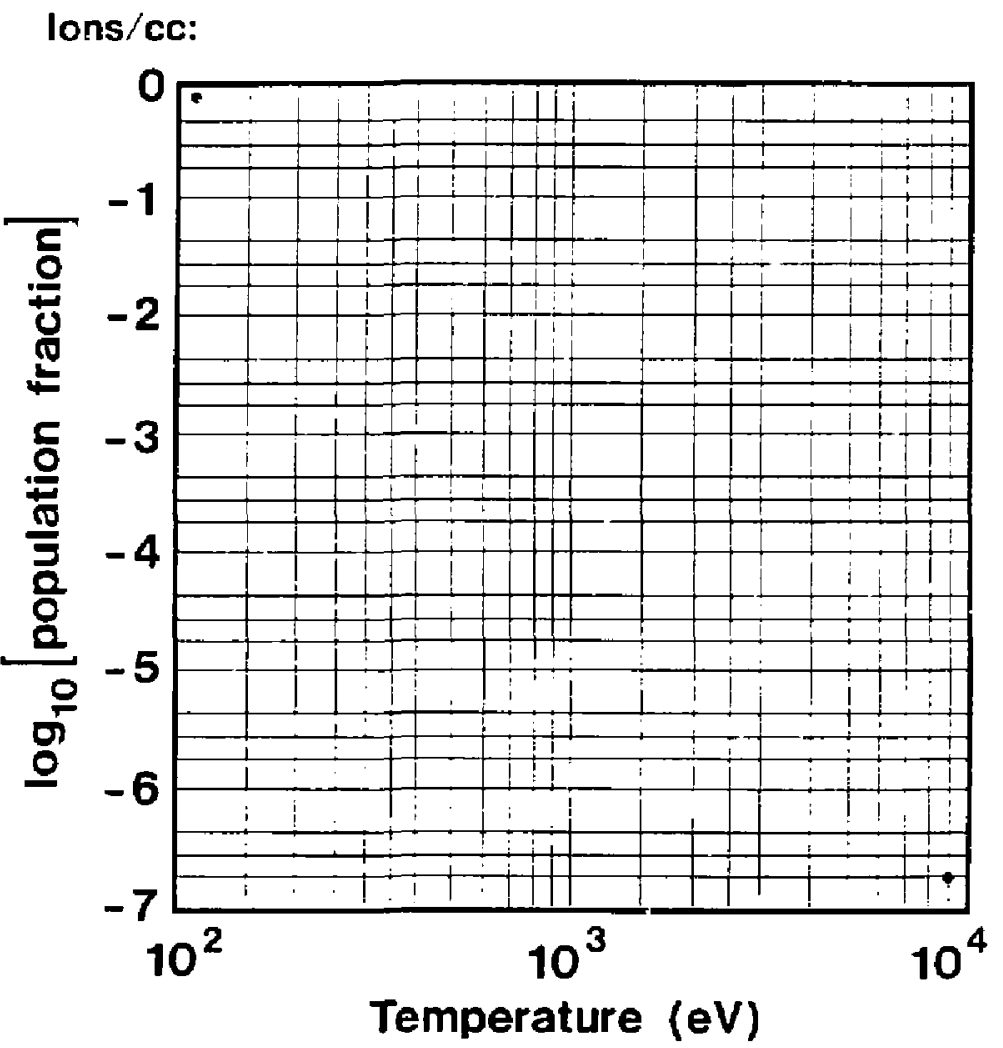

$$
\begin{aligned}
& 0 \text { - bare } \\
& 1 \text { - H-like } \\
& 2 \text { - He-like } \\
& 3 \text { - Li-like }
\end{aligned}
$$

Comparison of charge-state populations. As in Figs. 2 and 3, a log-log grid calibrates the curves on three transparent overlays, one for each of three codes. The codes are RATION, XRASER, and LINEZ. The optically thin argon plasma has $n_{i}=5.6 \times 10^{21}$ ions/cc. 


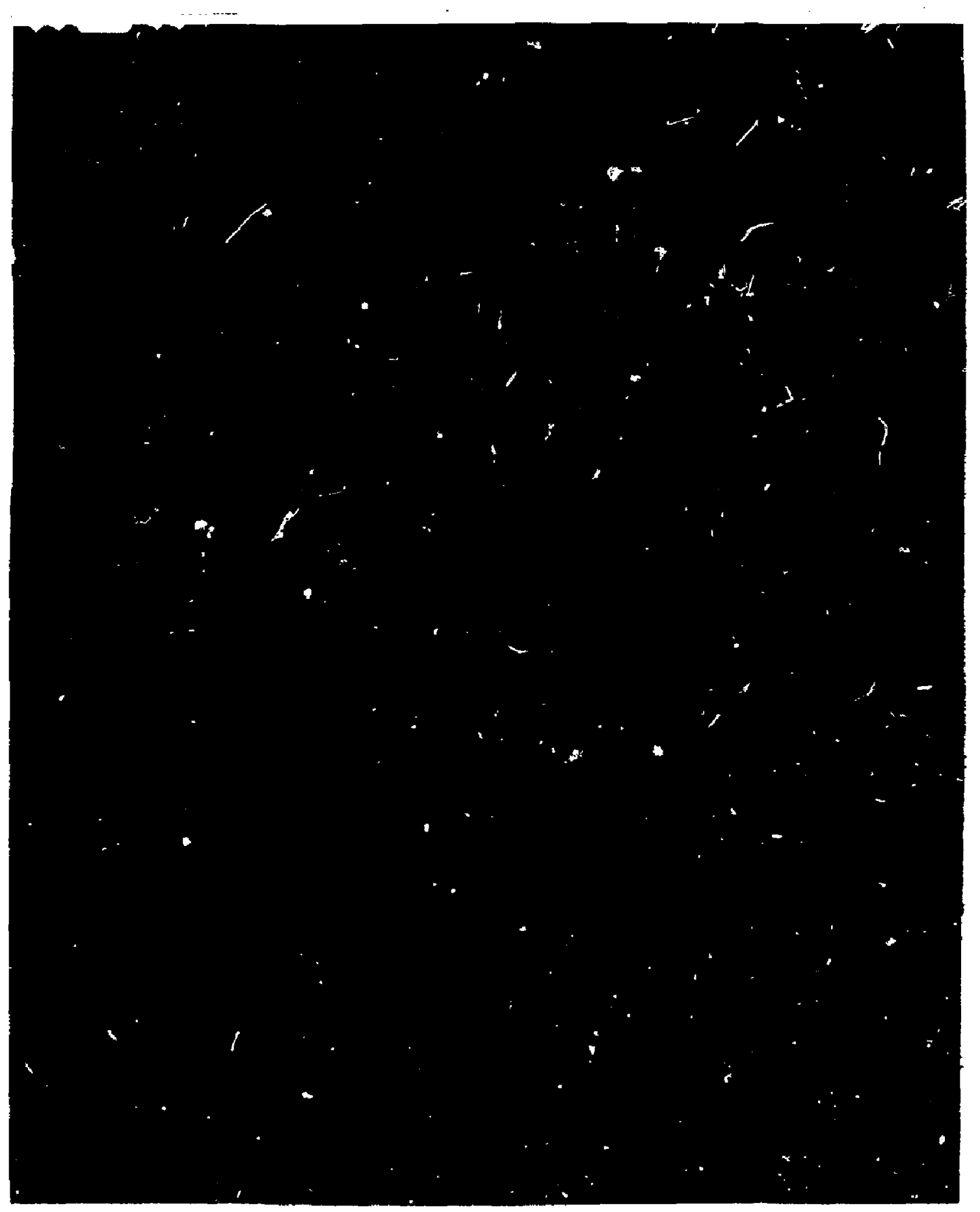



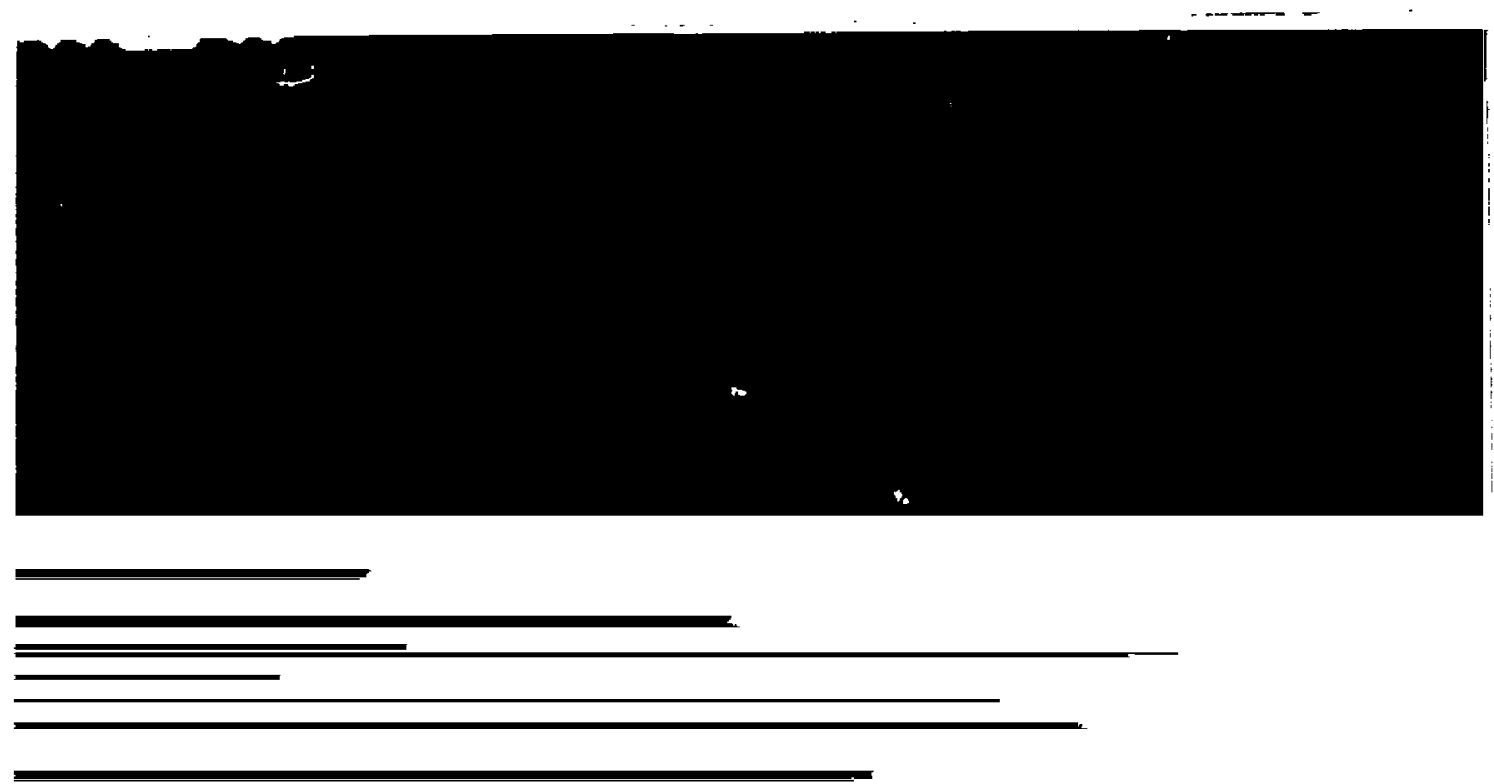

(20)

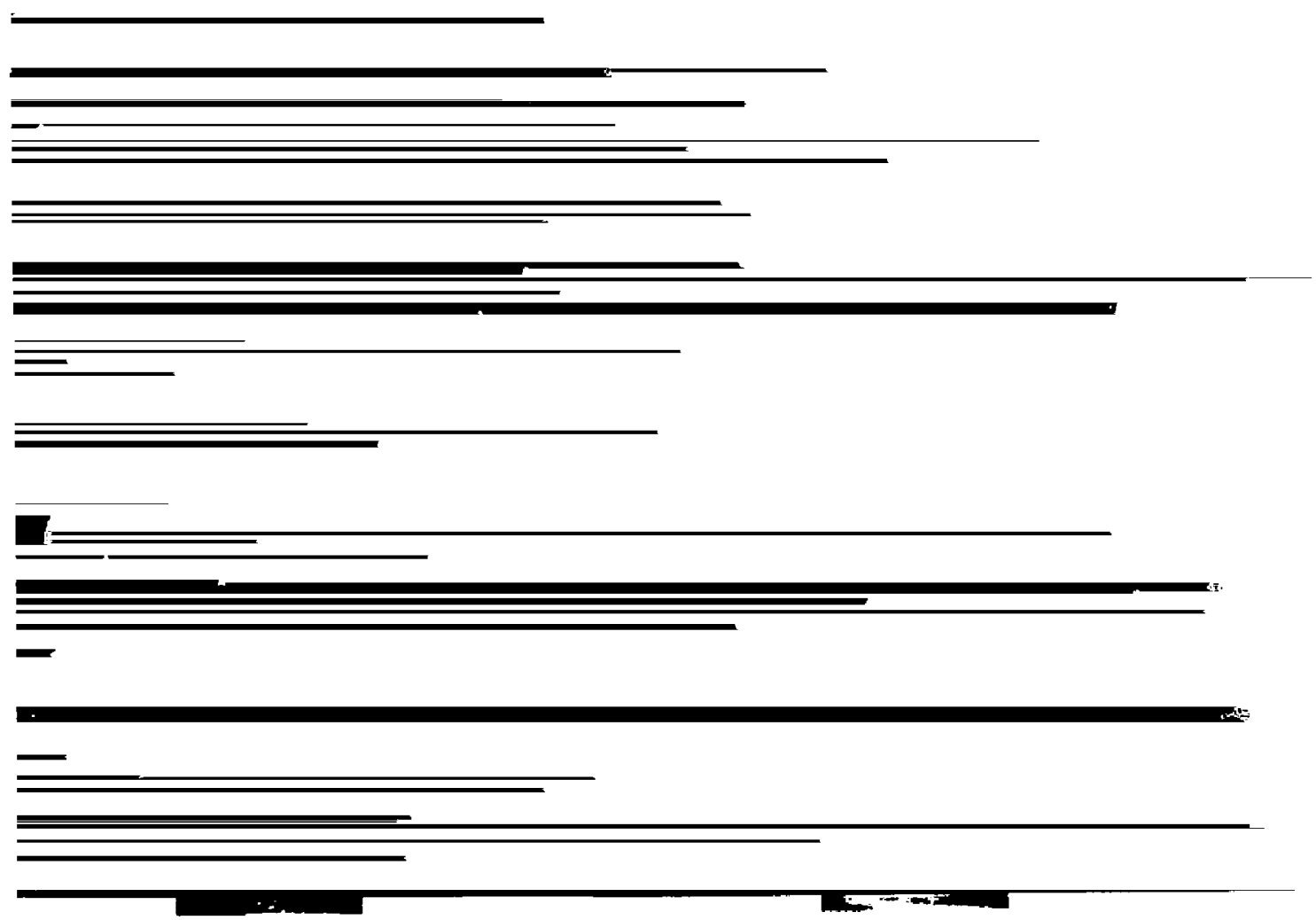




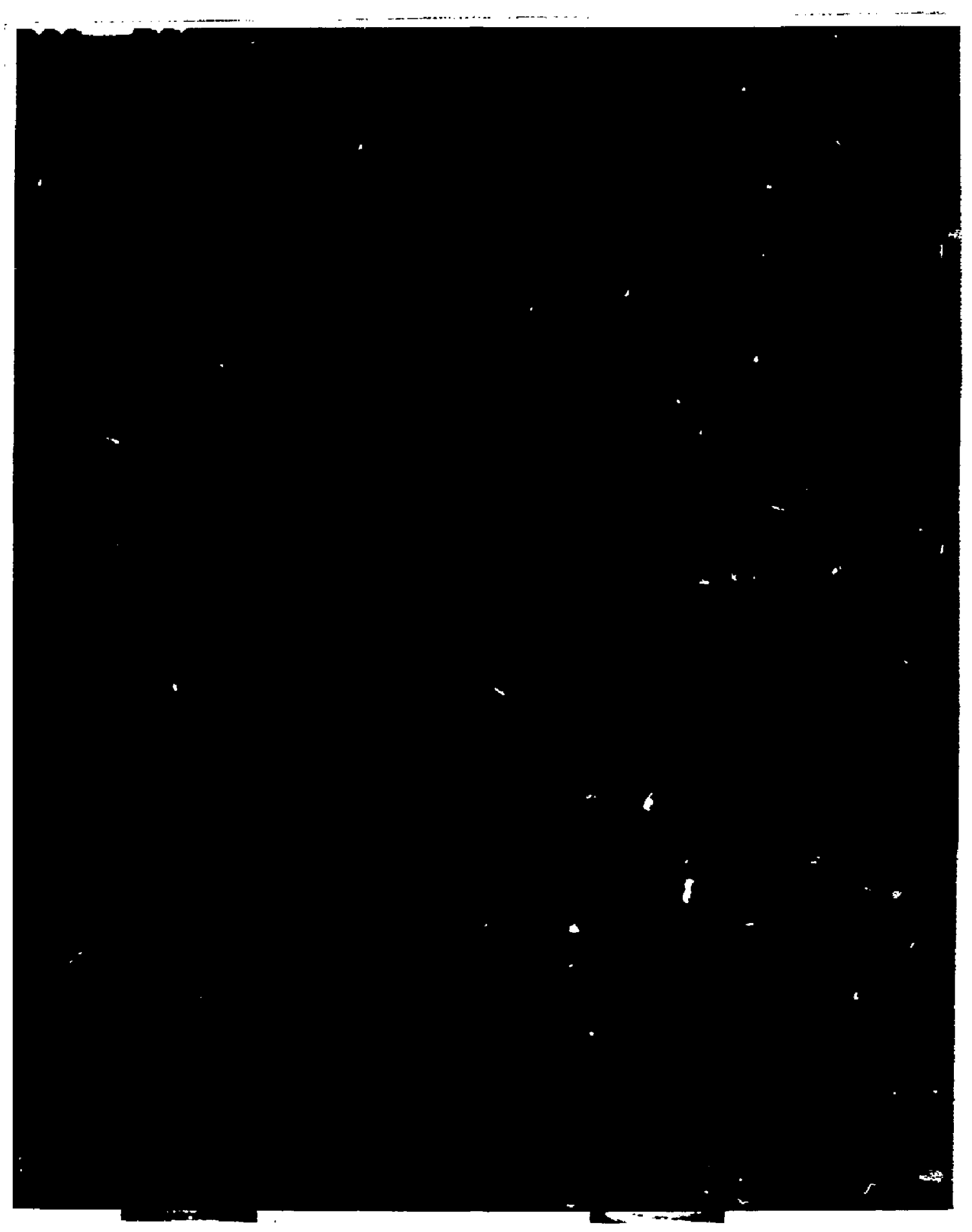




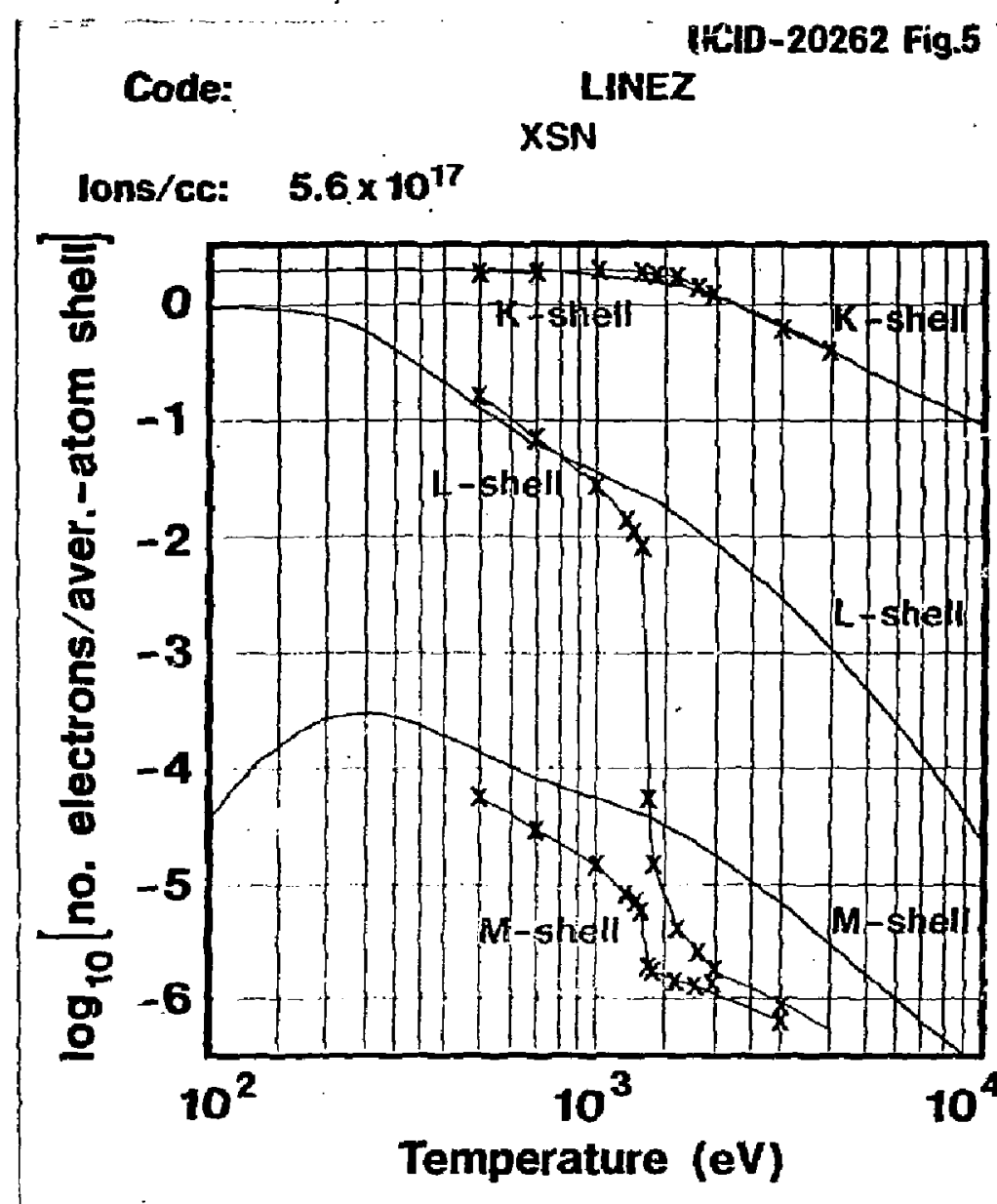

Numer of electrons in average-atom electronic shells. Election occupations are shown for the $K_{-}, L_{-}$and $M$-shells as functions of particle temperature. The average-atom code XSN is compared to LINEZ simulating an optically thin argon plasma. The $x$-marks are XSN data, and the solid curves are by LINEZ. Particle densities are $5.6 \times 10^{17}$ ions/cc and $10^{19}$ total electrons $/ c c$. 


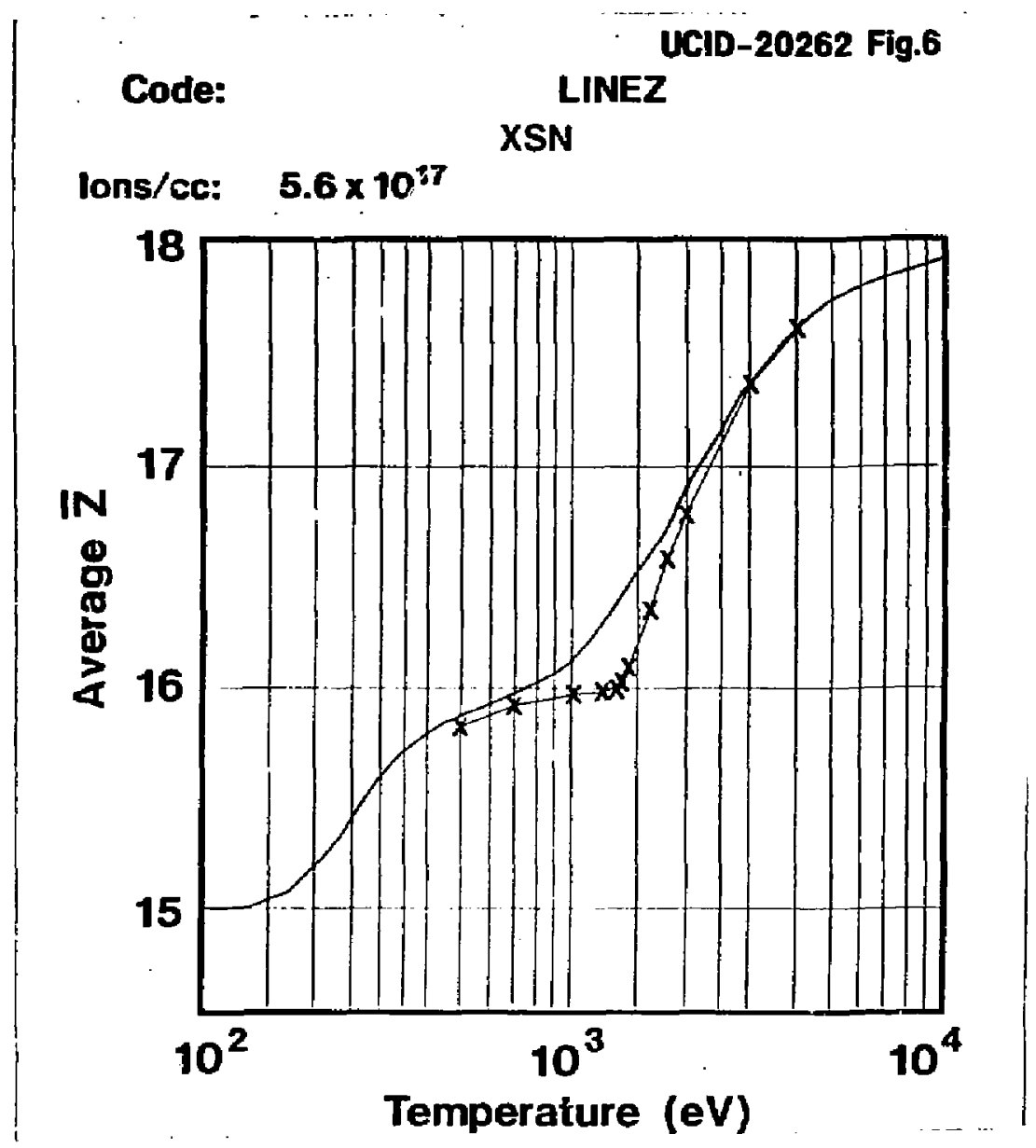

Average jonic charge $\bar{z}$ v.s, remperature. The average-atom code XSN is compared to LINEZ, both codes simulating an optically thin argon plasma. The $x$-marks are XSN data, and the solid curves are by LINEZ. Particle densities are $5.6 \times 10^{17}$ and $10^{19}$ total electrons/cc. 


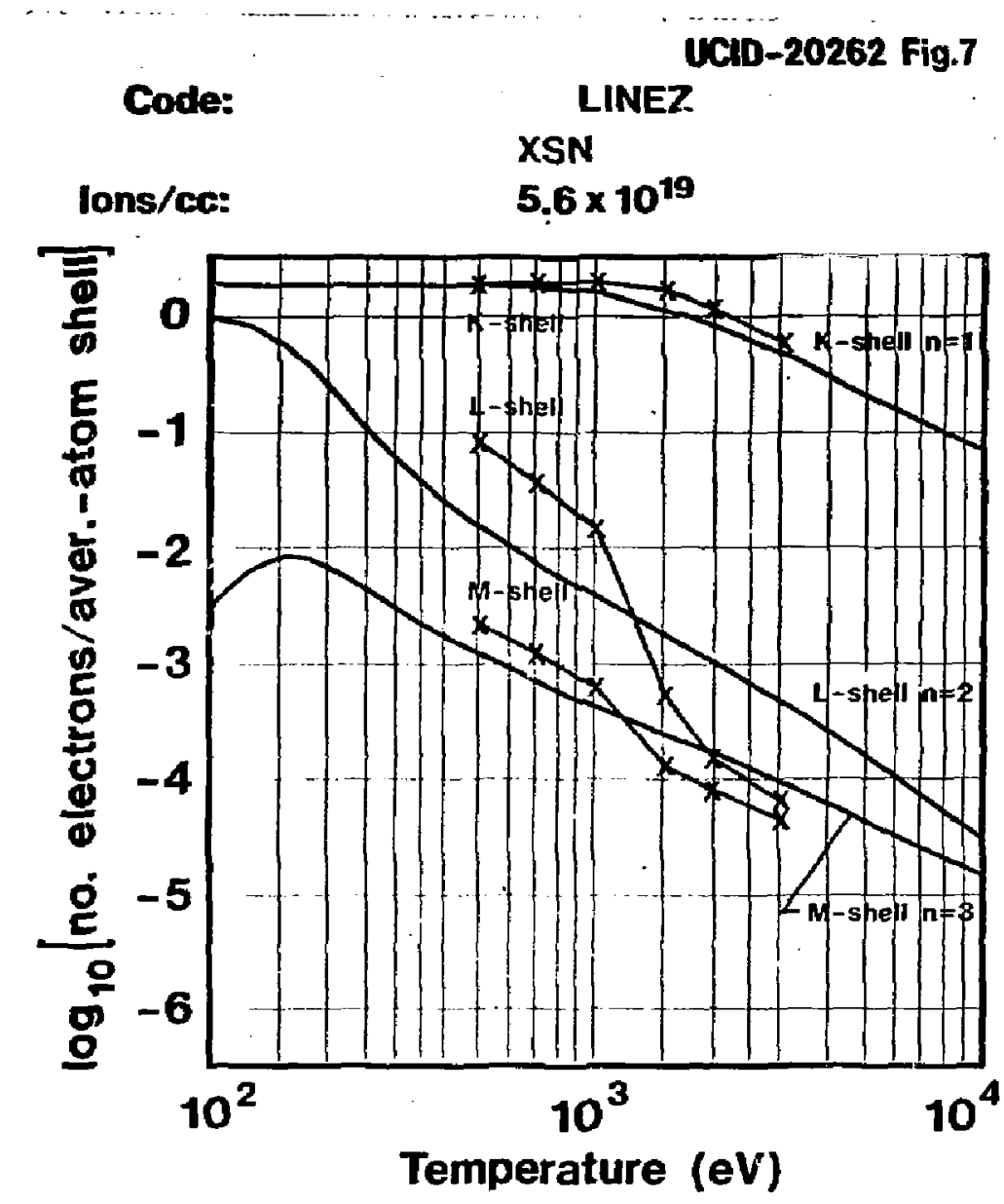

Number of electrons in average-atom electronic shells. Same as in Fig- 5 except that particle densities are $5.6 \times 10^{19}$ ions/ce and $10^{23}$ total electrons/cc. 


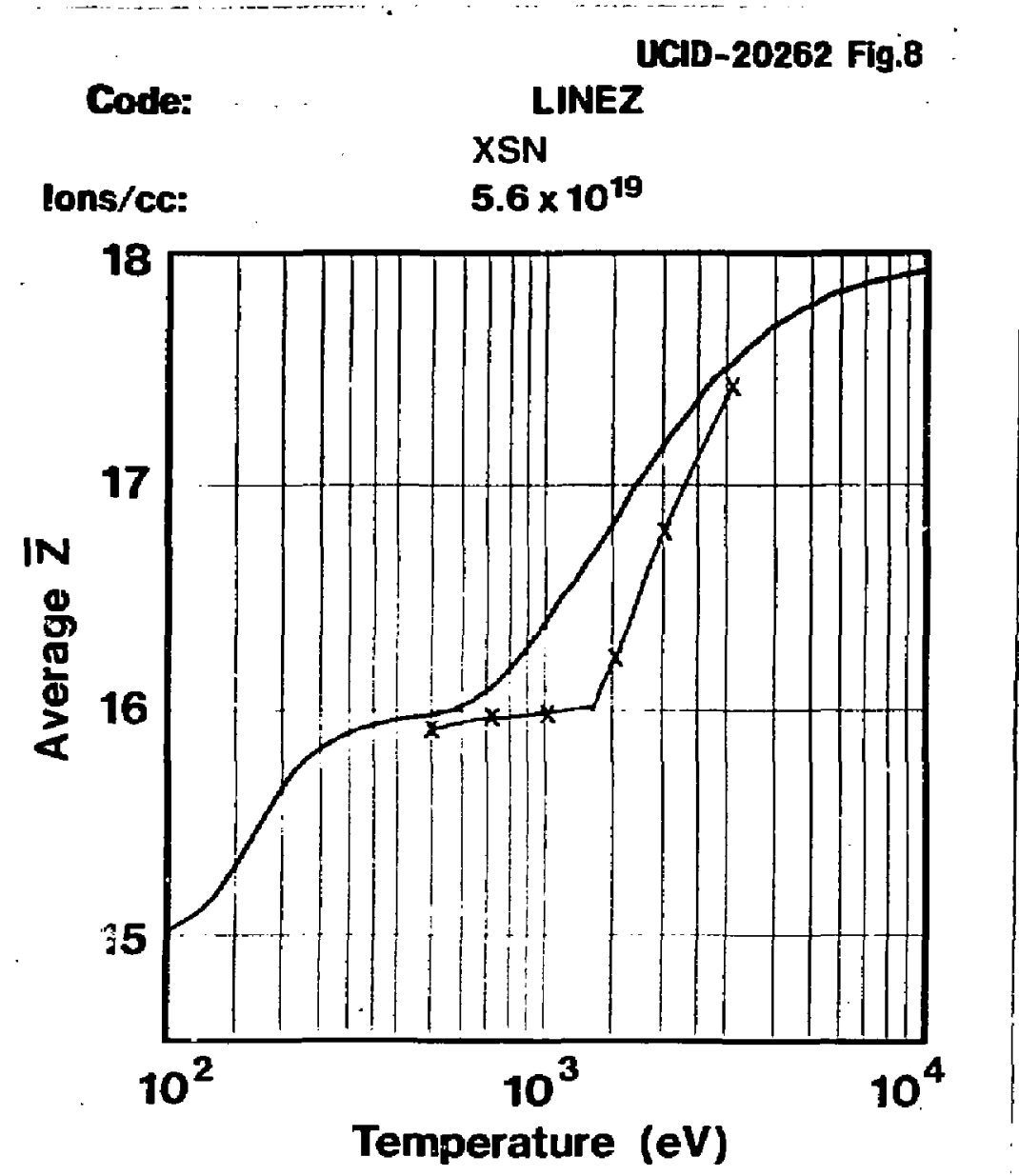

Average ionic charge $\bar{Z}$ v.s. temperature. Same as in Fig. 6, except that particle densities are $5.6 \times 10^{19}$ ions/ce and $10^{21}$ total electrons/cc. 


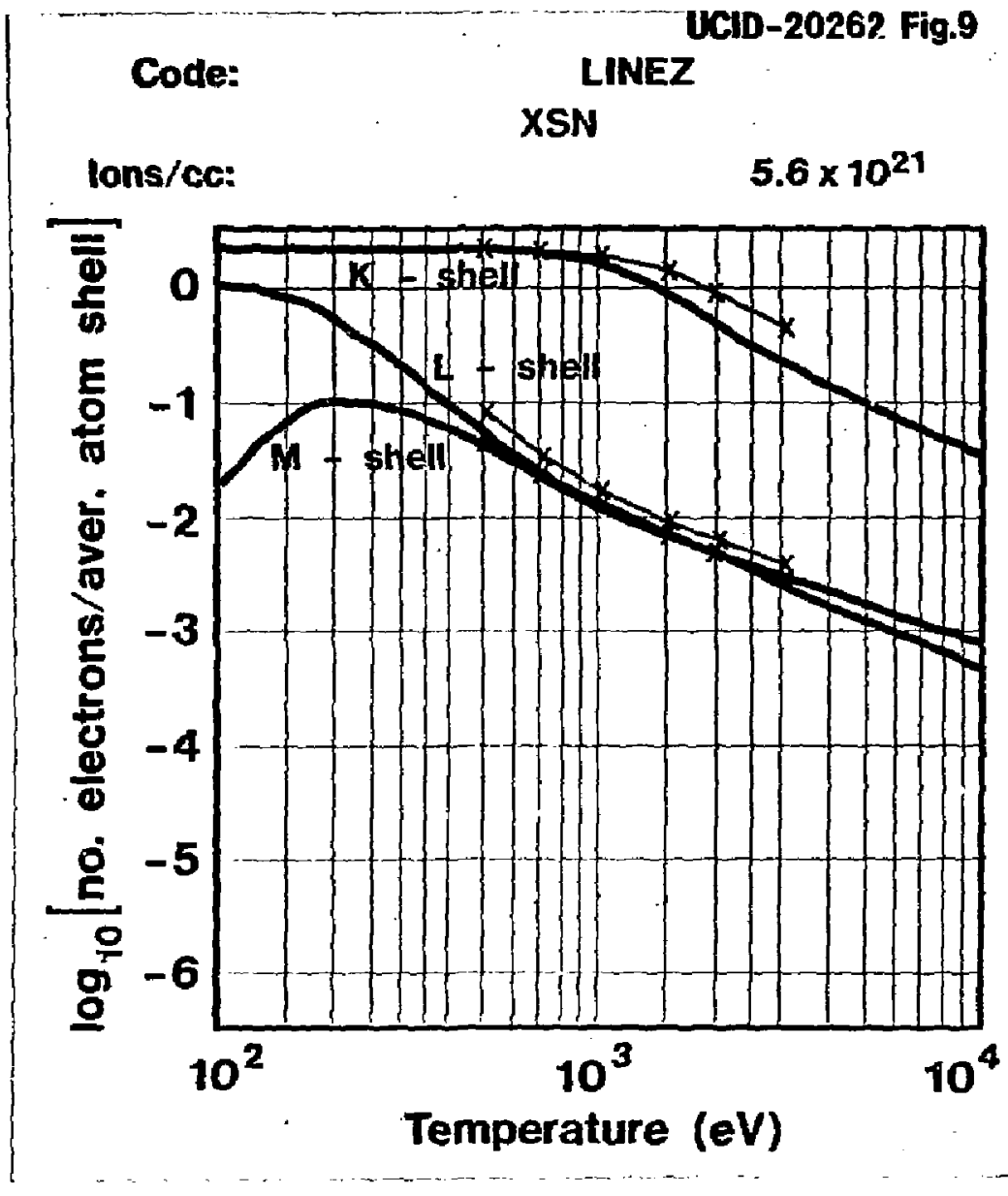

Number of electrons in average-atom electronic shells. Same as in Figs. 5 and 7 , except that particle densities ate $5,6 \times 10^{21}$ jons/ce and $10^{23}$ total electronsic $c_{\text {. }}$ 


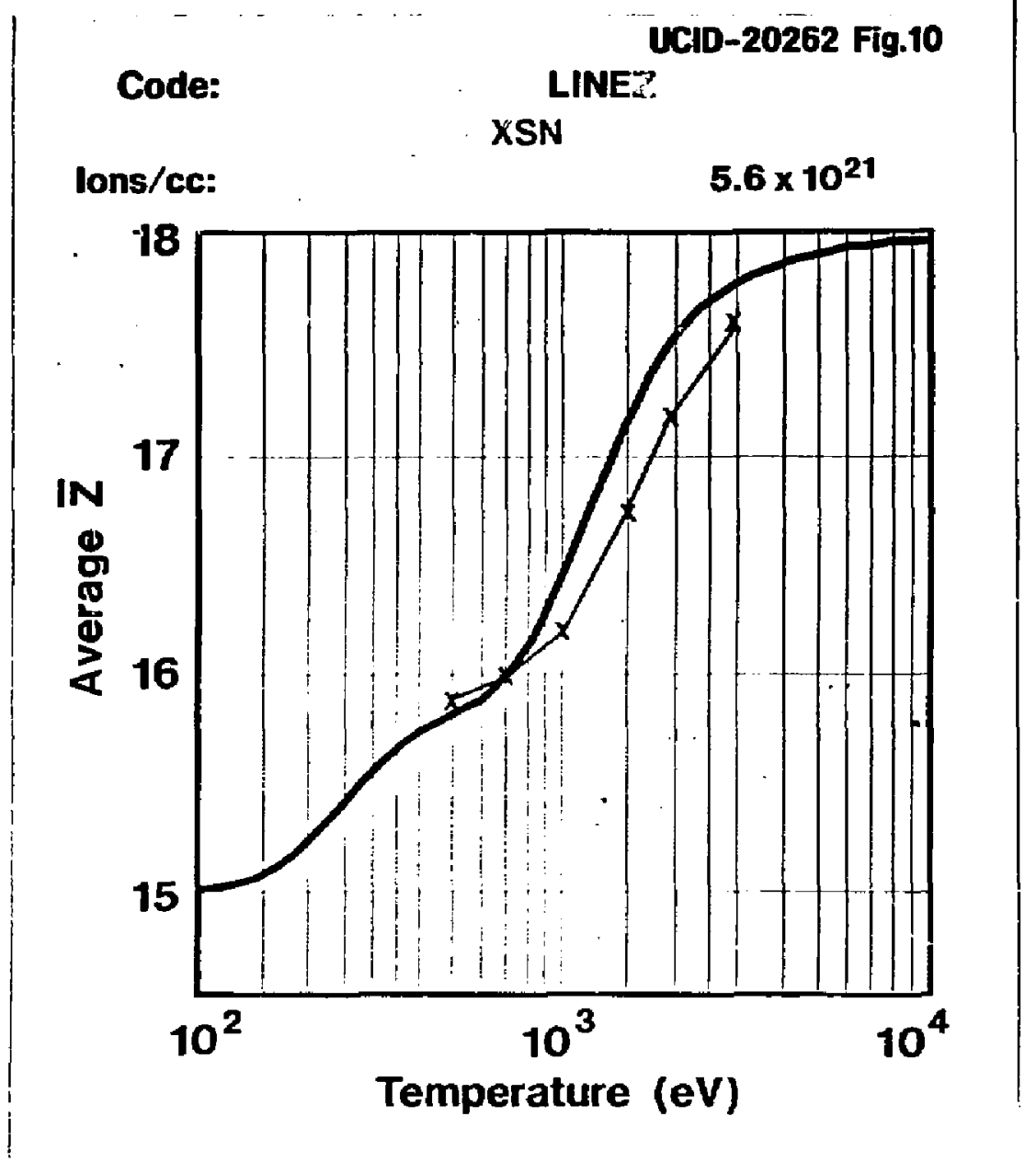

Average ionic charge $\bar{Z}$ v.s. temperature. Same as in Figs. 6 and 8 , except that particle densities are $5.6 \times 10^{21}$ ions/cc and $10^{23}$ total electrons/cc. 
Code:

lons/ce:

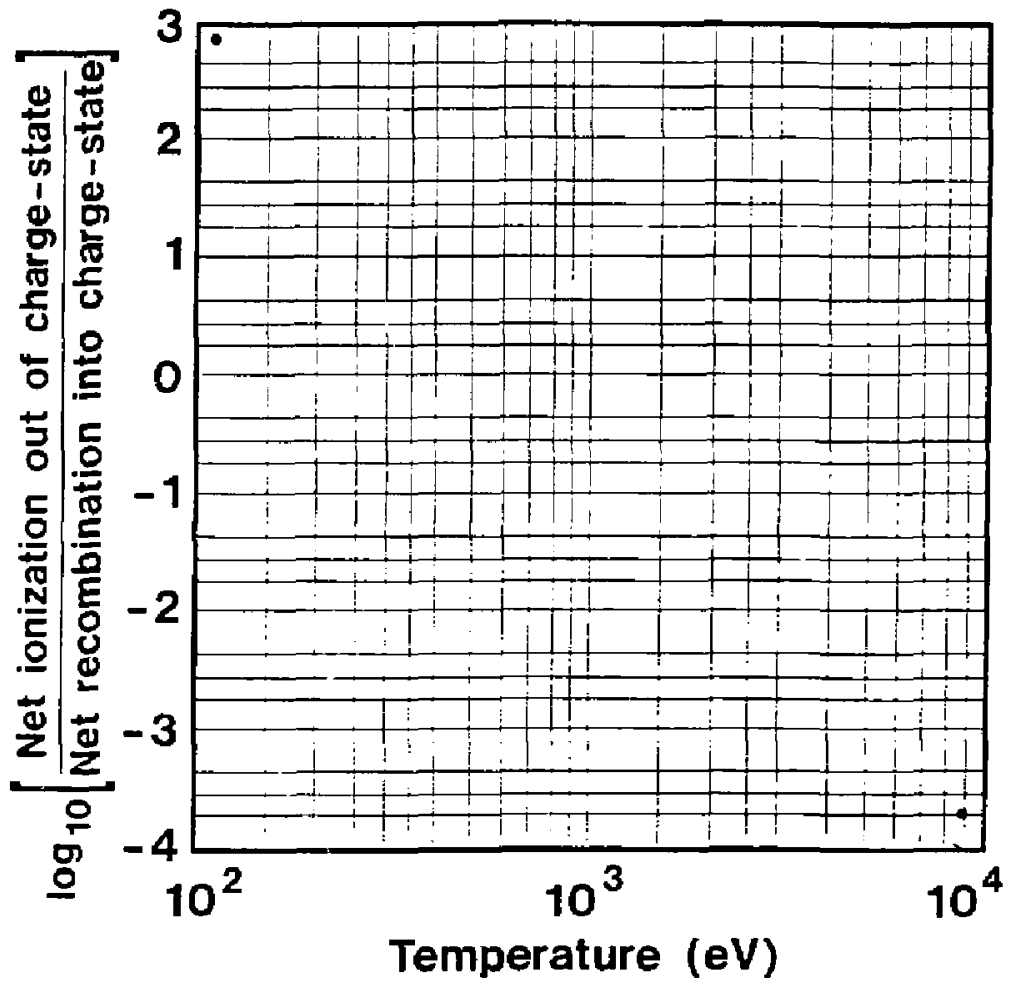

1 - H-like

2 - He-like

3 - Li-like

Ratios of [net ionization out of] / [net recombination into] each ionic charge-state of an optically thin argon plasma. A single grid calibrates the axes, while separate transparent overlays, one for each NLTE code, show the three ratios computed. The codes are RATION, XRASER, LINEZ, YTL, FOREST, and NRL. The argon plasma is simulated at particle densities of $5.6 \times 10^{19}$ ions/ce and $10^{21}$ total electrons/cc. 


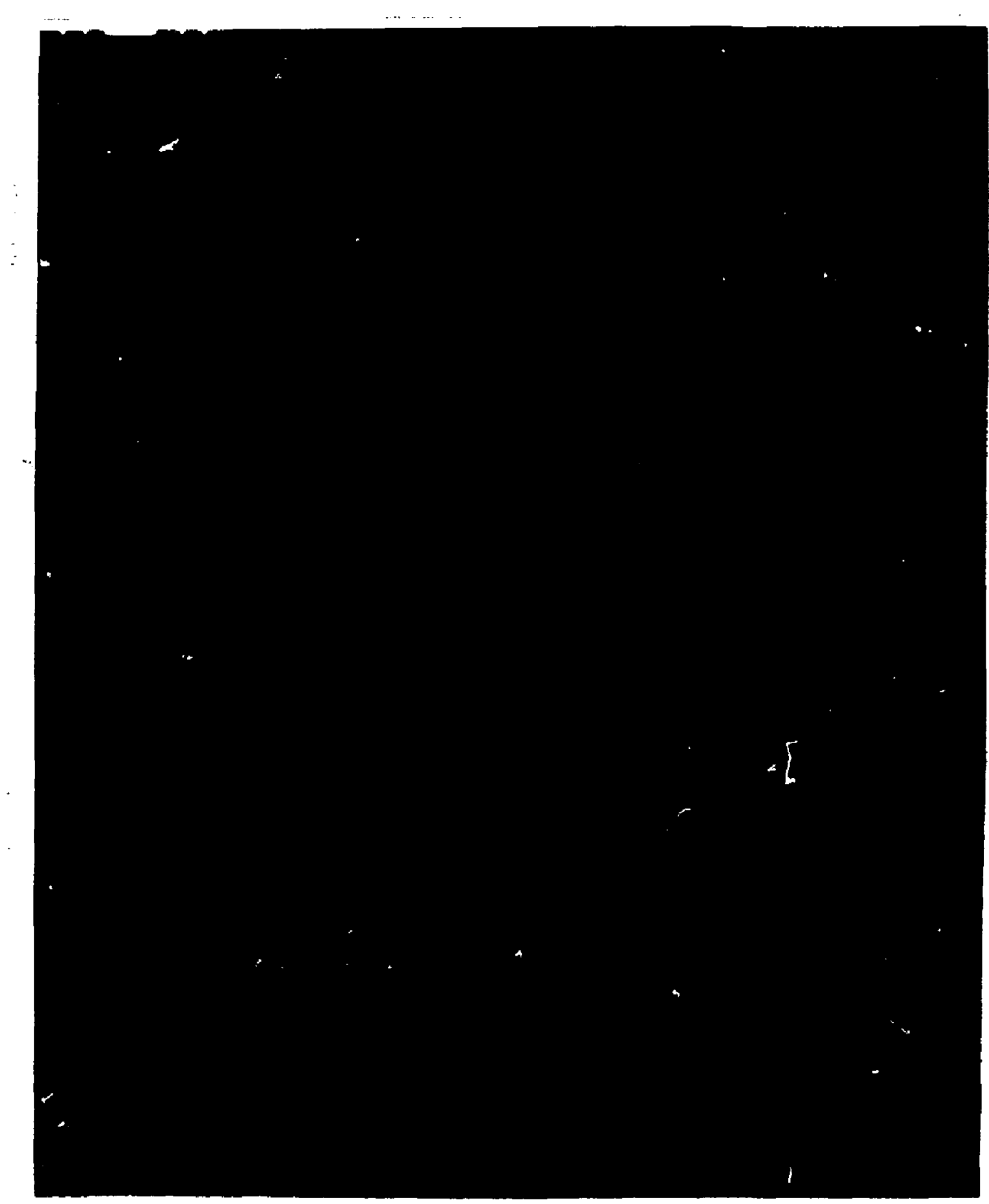


n

$+$
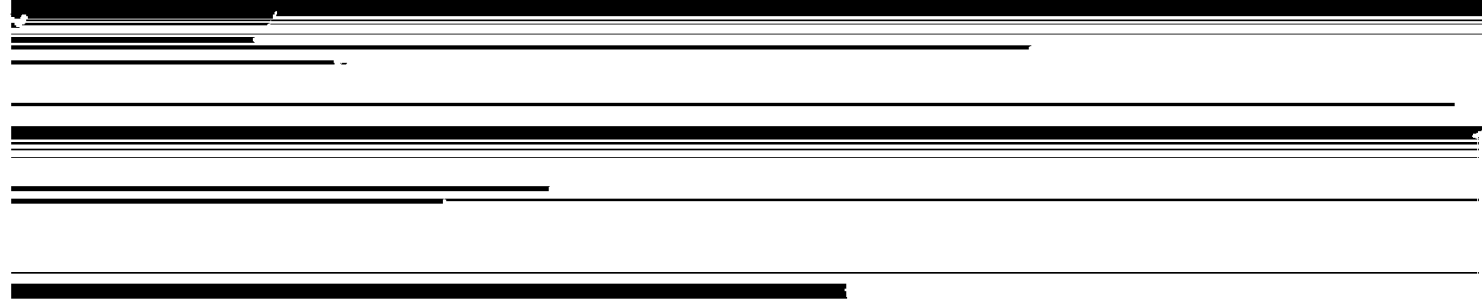


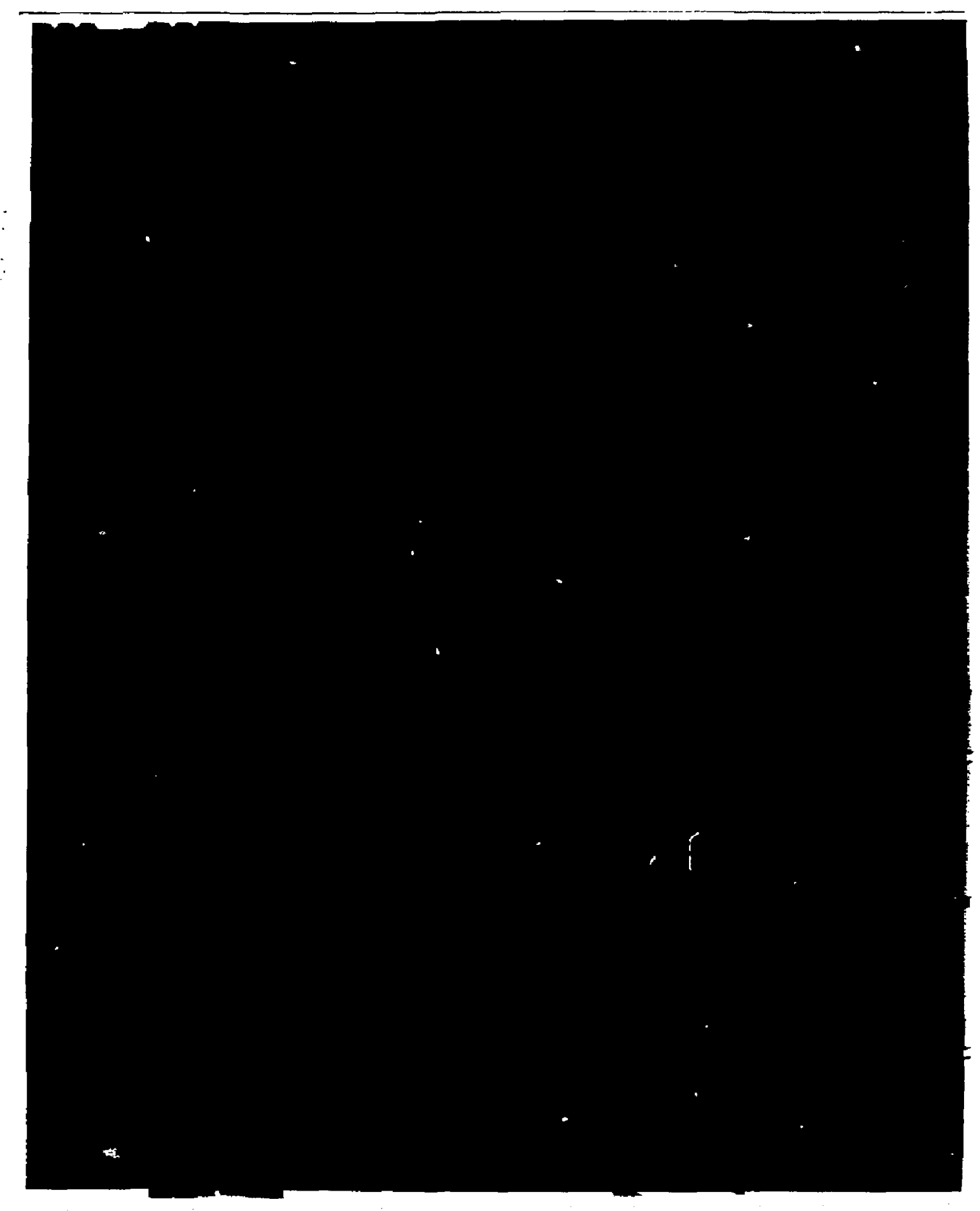


UCID-20262 Fig.i1D YTL

$5.6 \times 10^{19}$

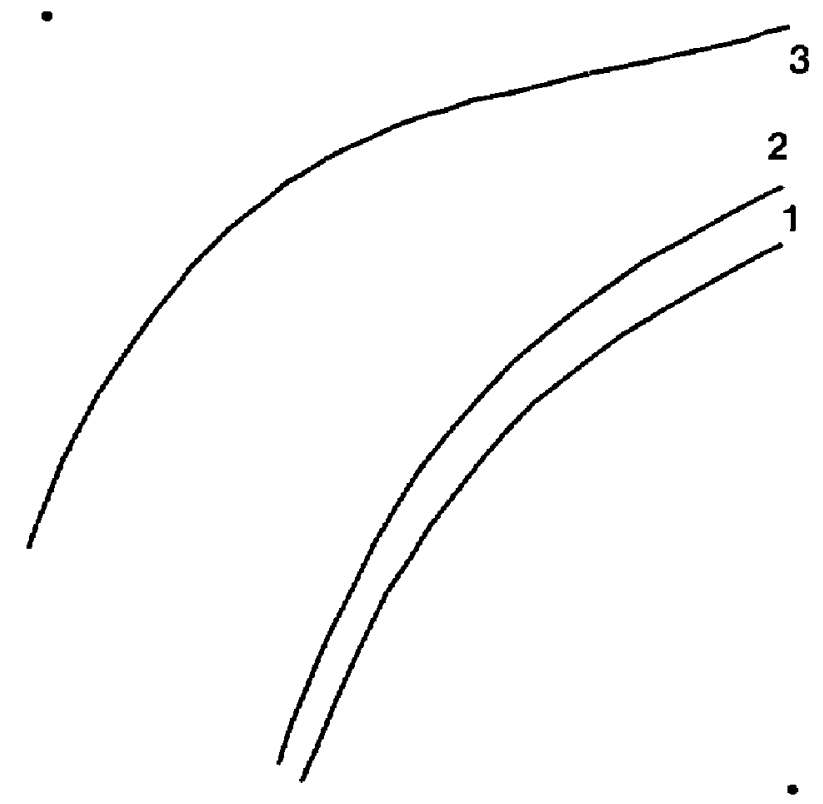




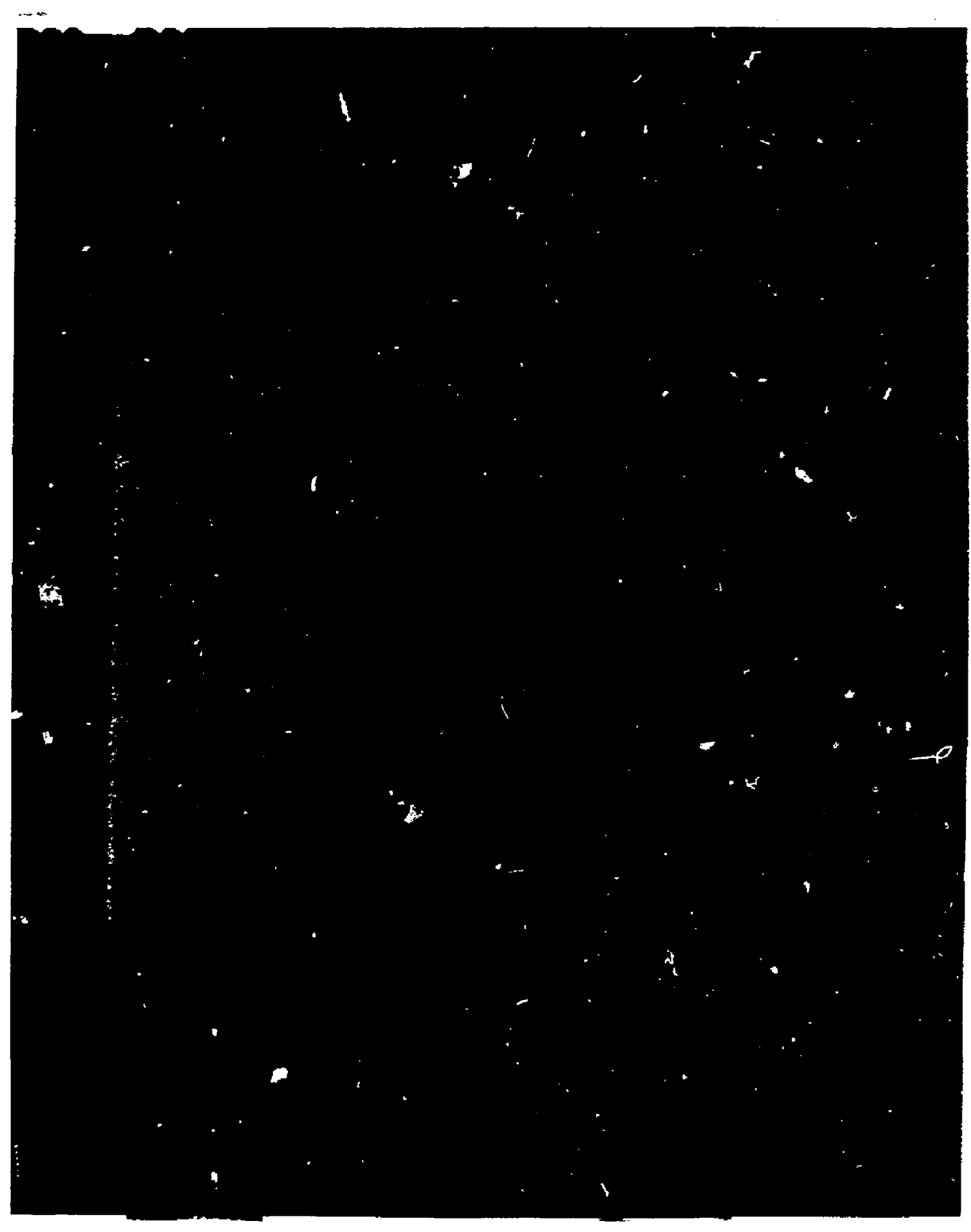




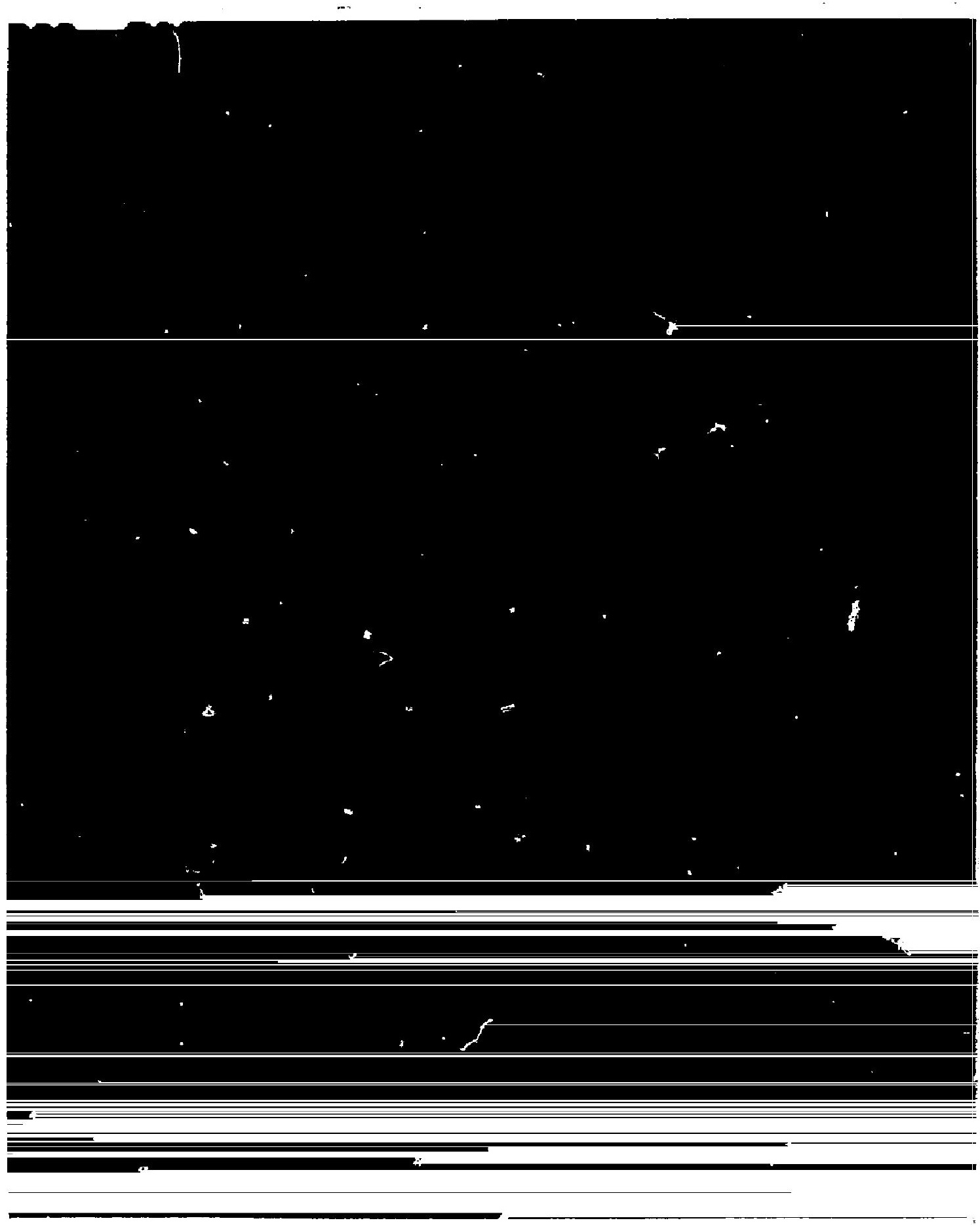




\section{Code:}

\section{Ions/cc:}

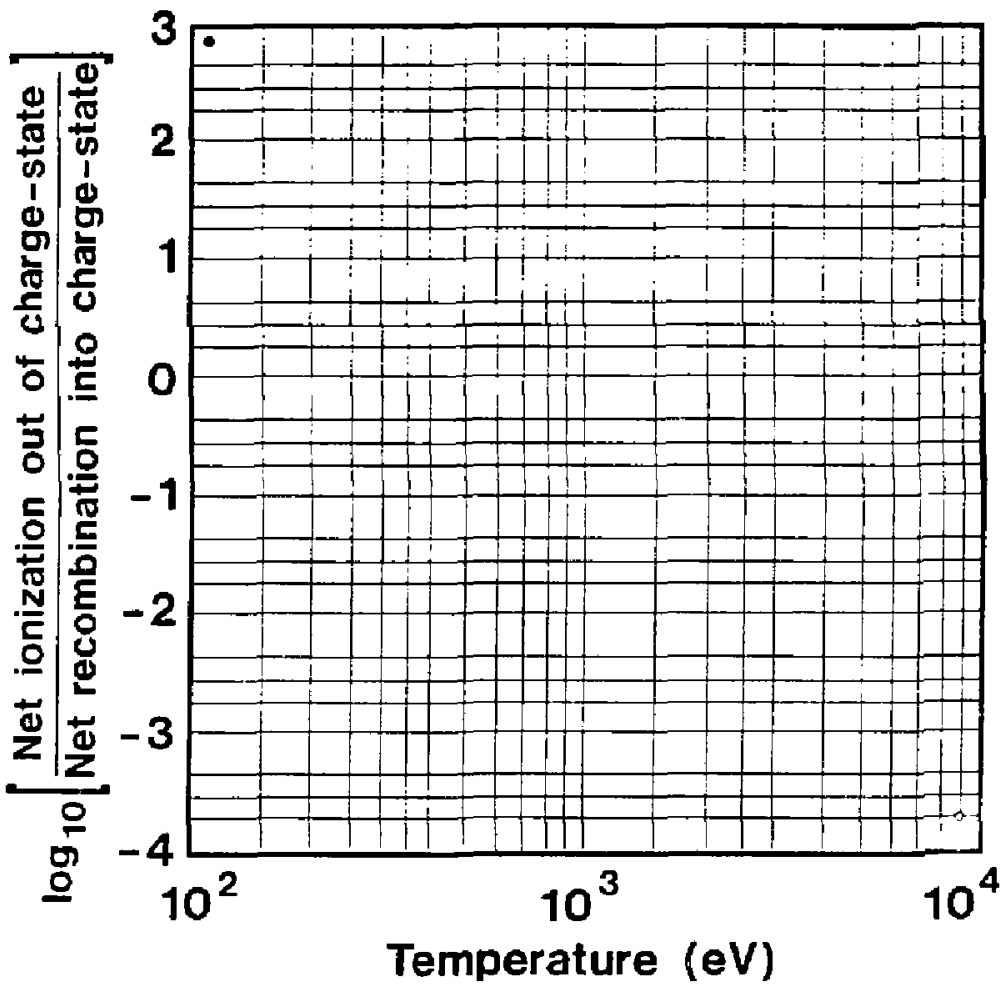

1 - H-like

2 - He-like

3 - Lj-like

Ratios of [nel ionization out of] / [net recombination into] each charge-state of an optically thin Ar plasma. The codes are the same as in Fig. 3: RATION, XRASER, LJNEZ, NEBULA, and PPL. The plasina is simulated at particle densities of $5.6 \times 10^{17} \mathrm{ion} / \mathrm{cc}$ and $10^{19}$ total electrons/ce. 


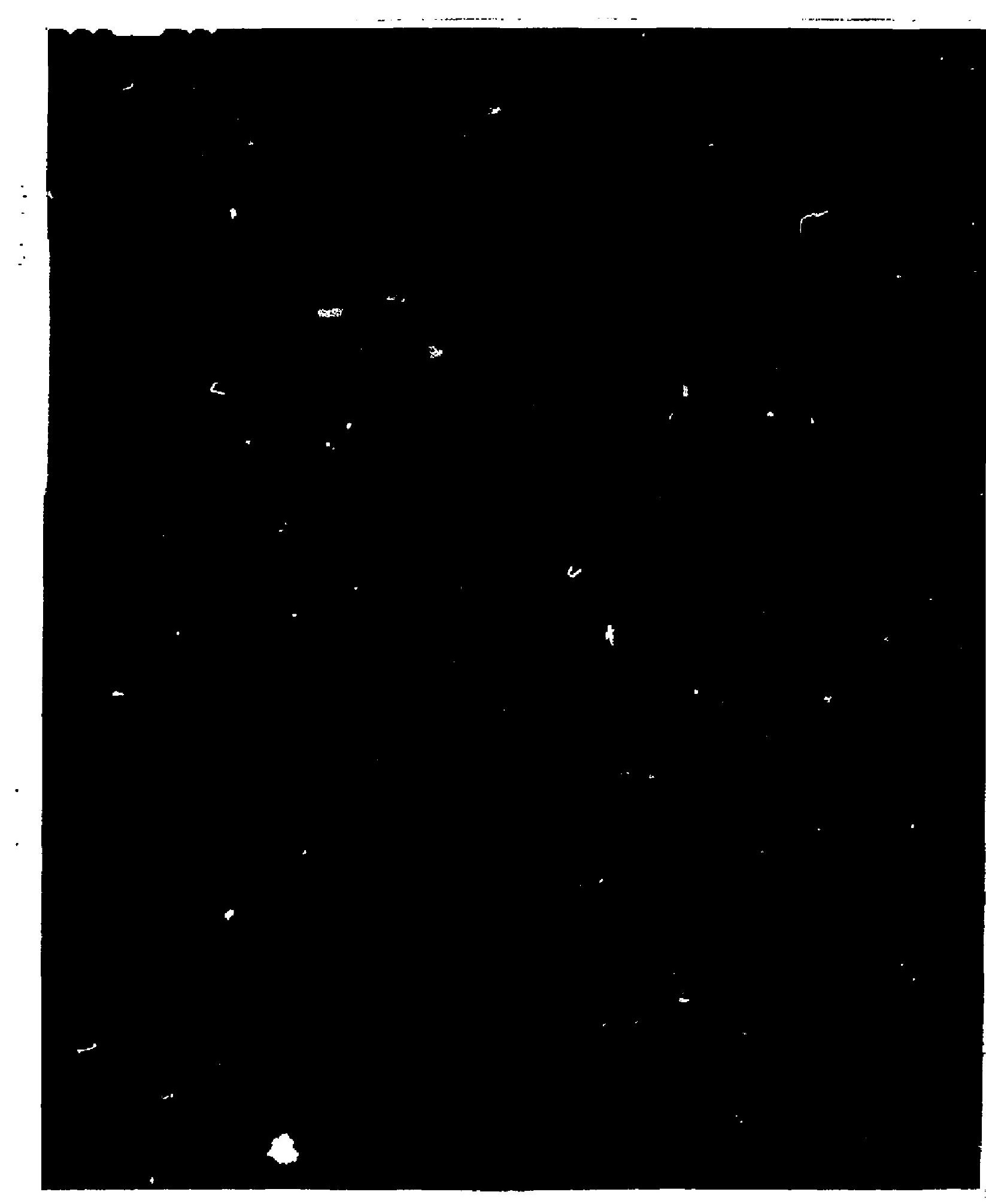




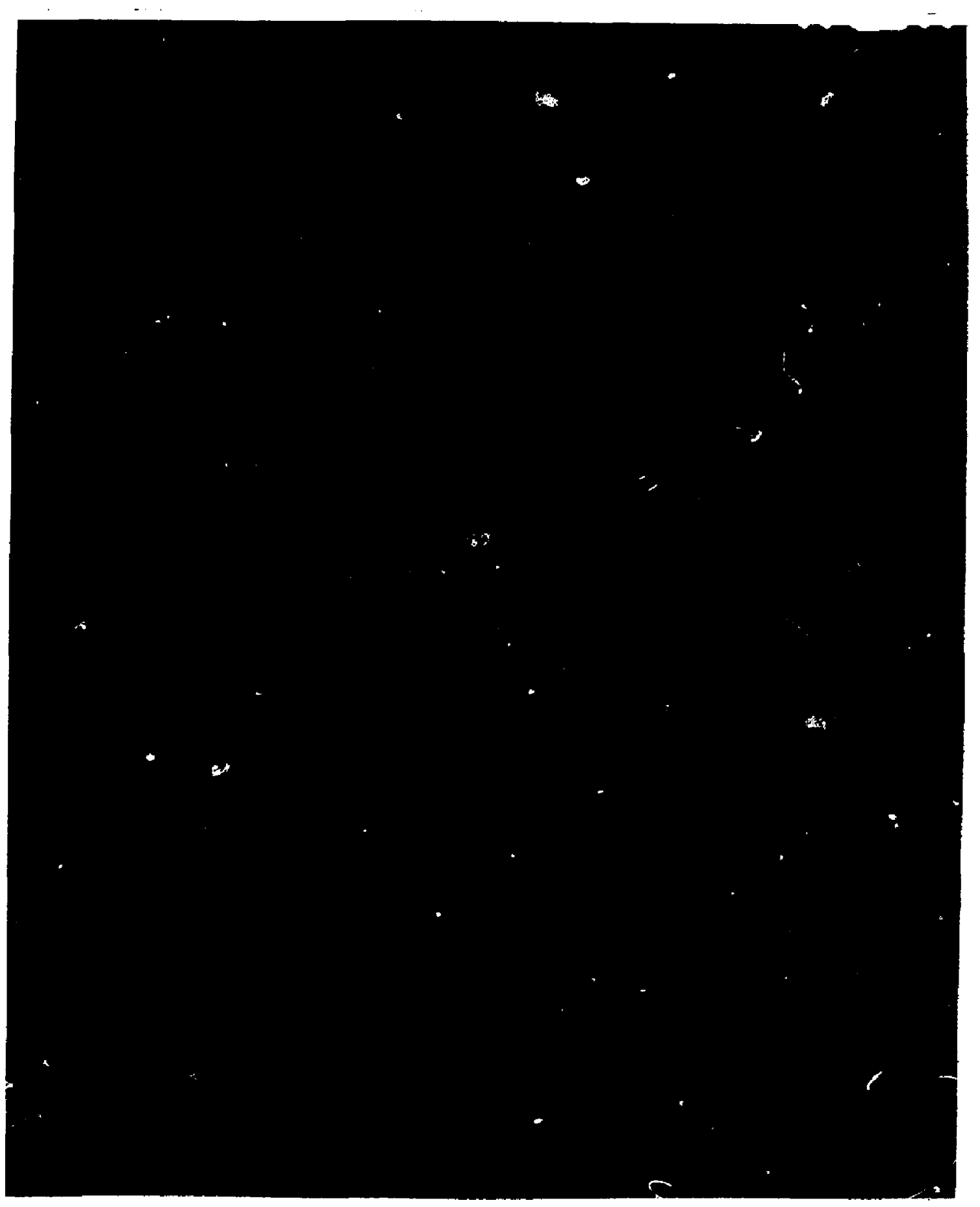




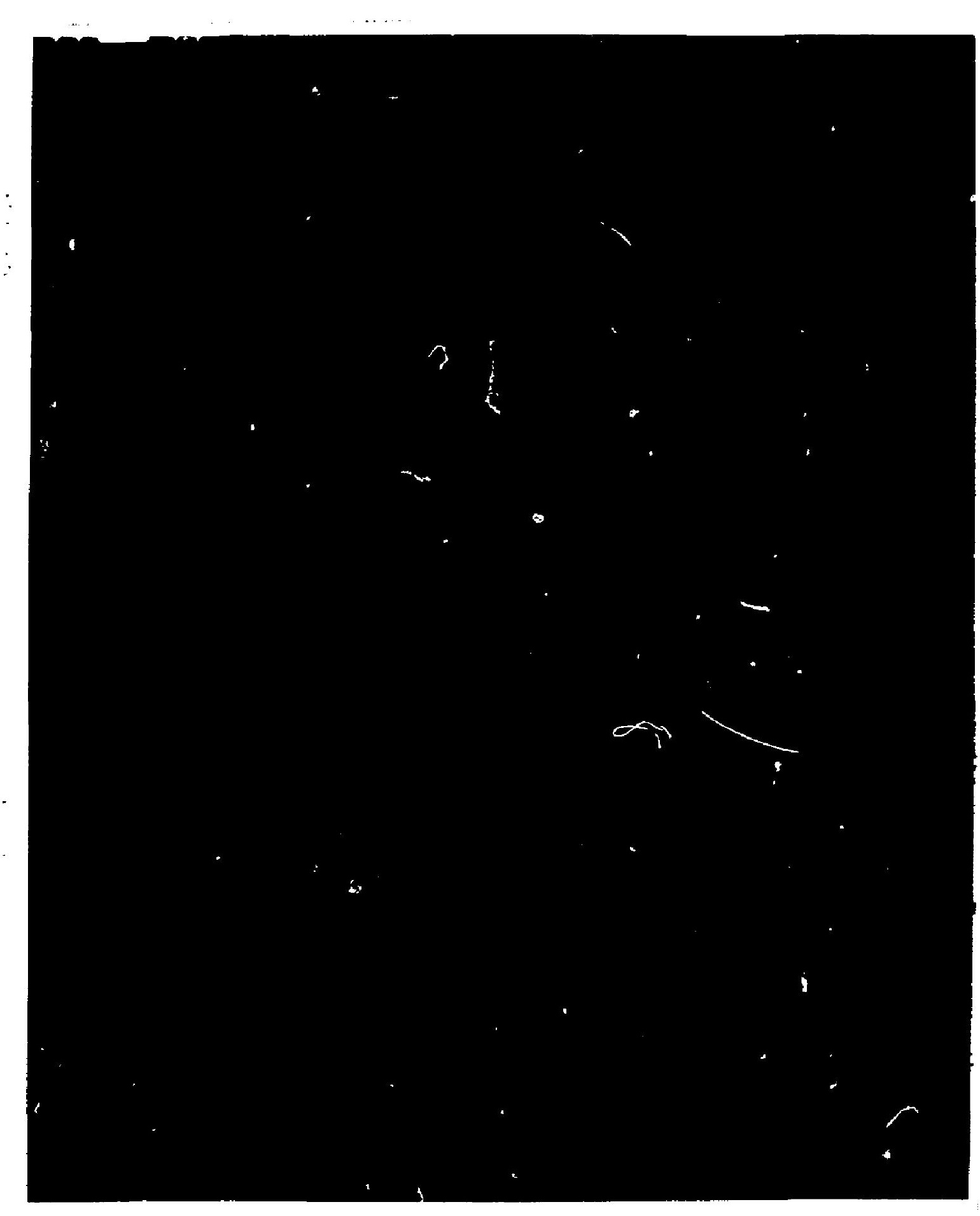




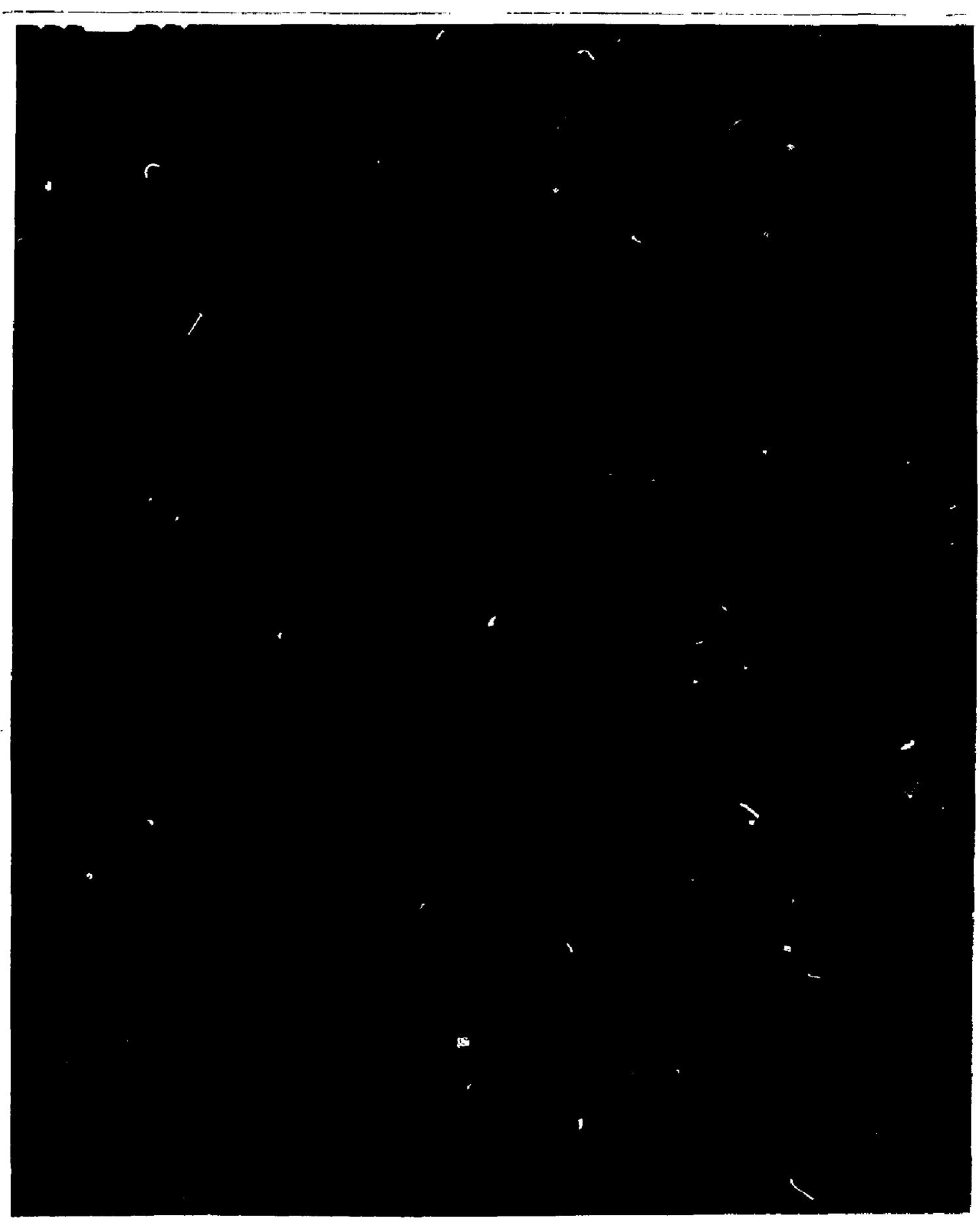



Code:

\section{Ions/cc:}

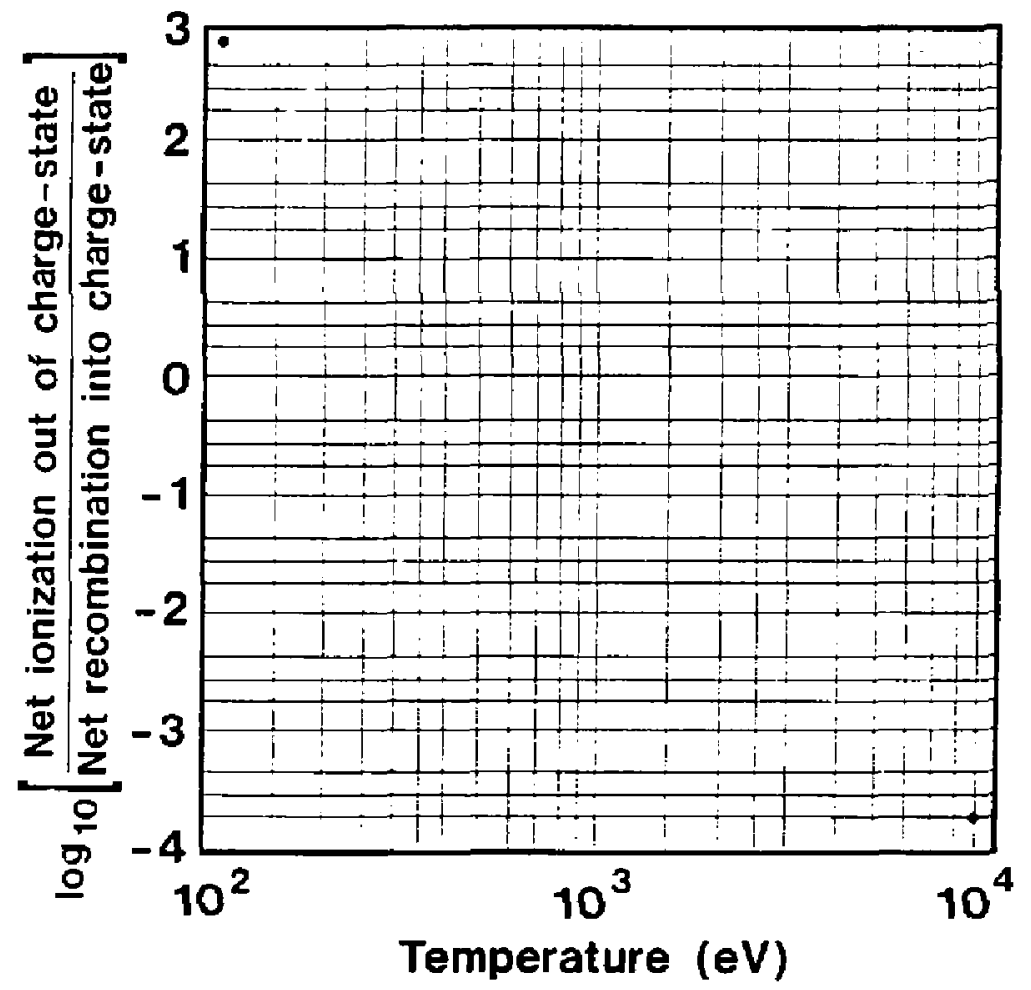

1 - H-like

2 - He-like

3 - Li-like

Ratios of [net iorization out of] / [net recombination into] each charge-state of an optically thin Ar plasma. The codes are the same as in Fig. 4: RATION, XRASER, and LINEZ. The particle densities are $5.6 \times 10^{22}$ ions/ce and $10^{23}$ total electrons/cc. 


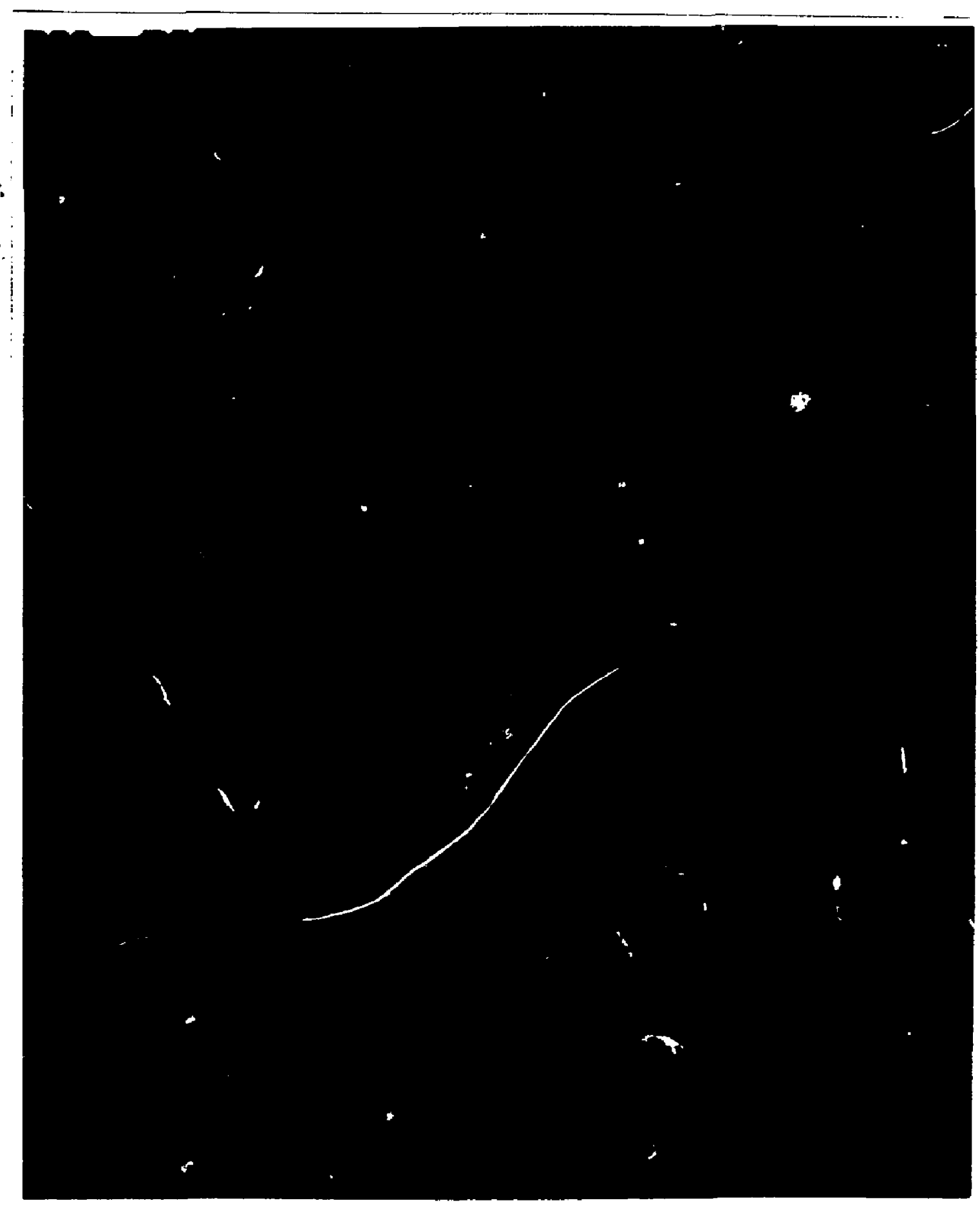




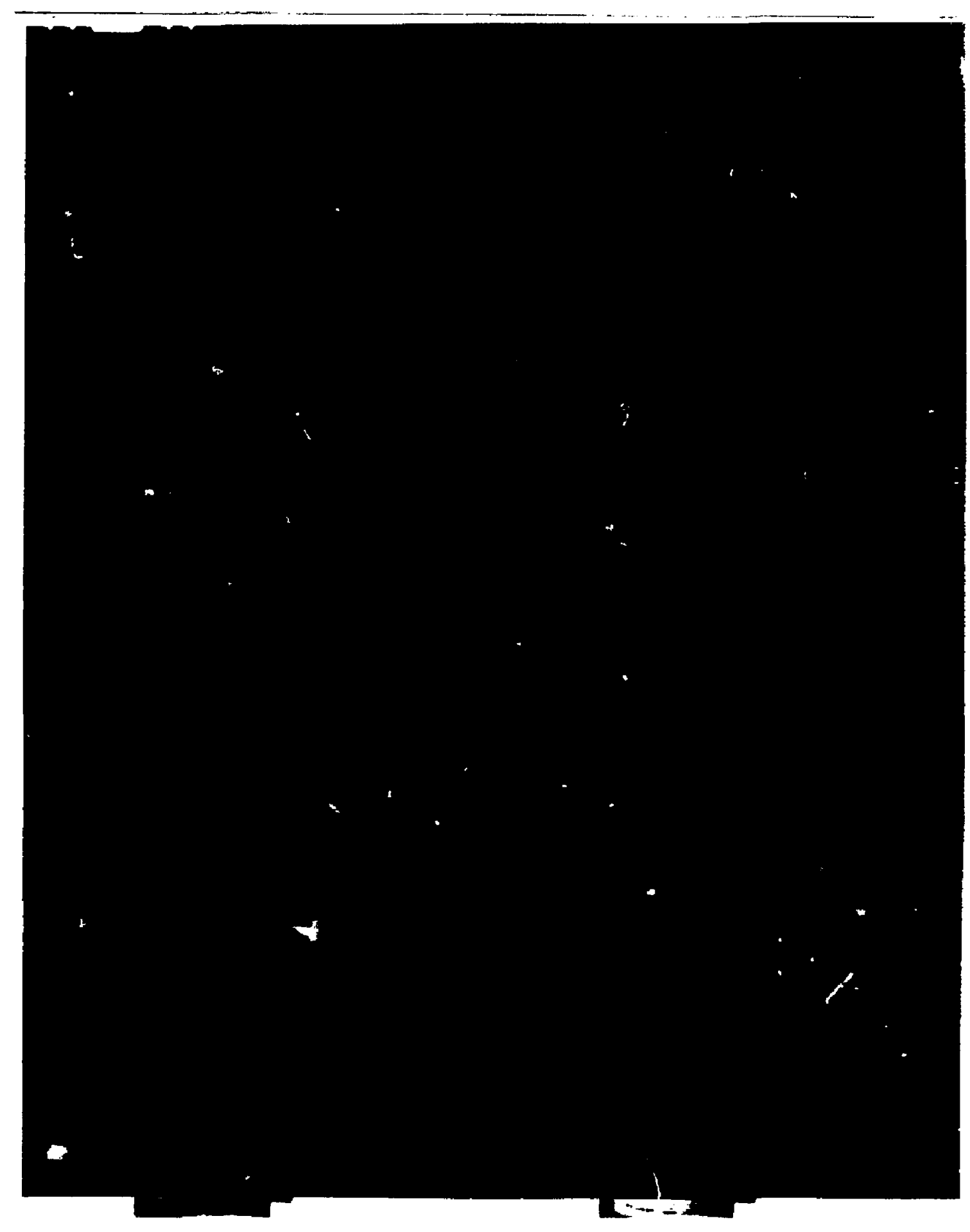




\section{Thers}
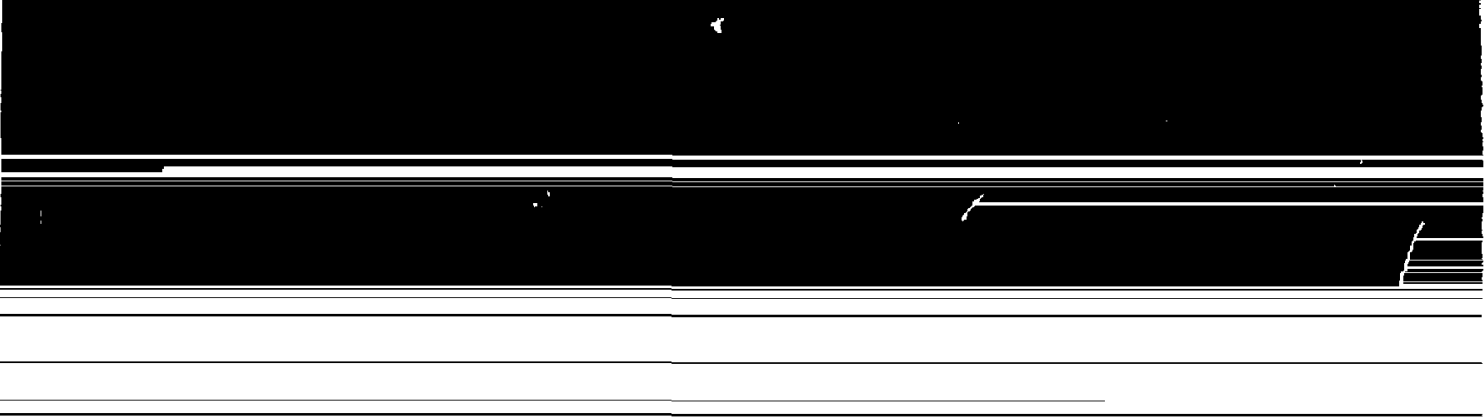

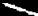

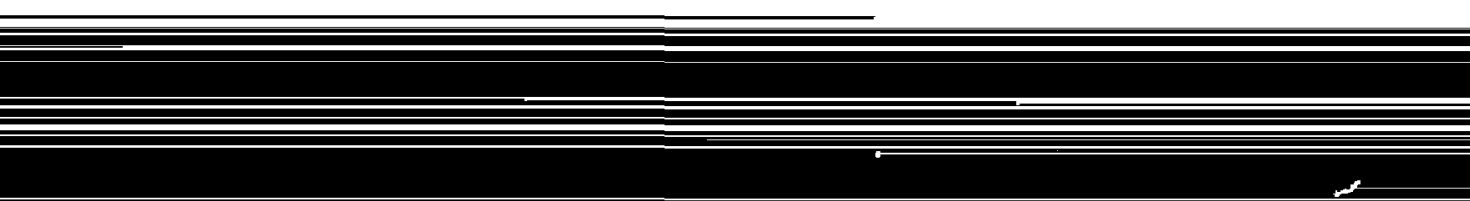

เ

$\leftarrow$

,

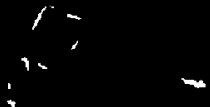

4

$-$ 


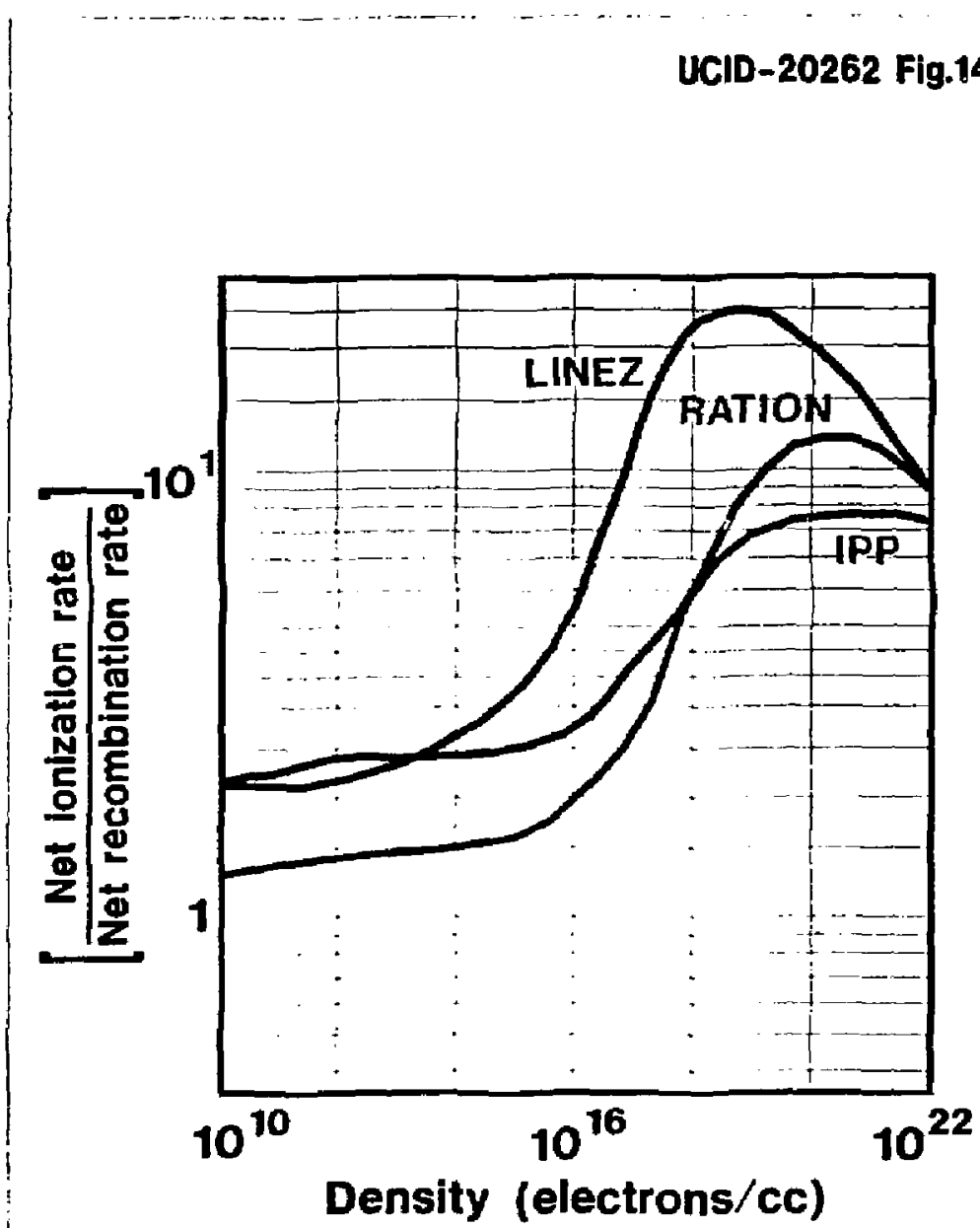

Ratios of [net jonization out of] / [net recombination inta] the ground state of He-like 0 VII in an optically thin oxygen plasma. The code IPP is compared to RATION and to LINEZ. Particle density varies 12 orders of magnilude, while temperature is constant at $172 \mathrm{eV}$. 


\section{- Codes unEZ \\ lons/ce: $5.6 \times 10^{19}$ \\ Strength: Normal Half-strength}

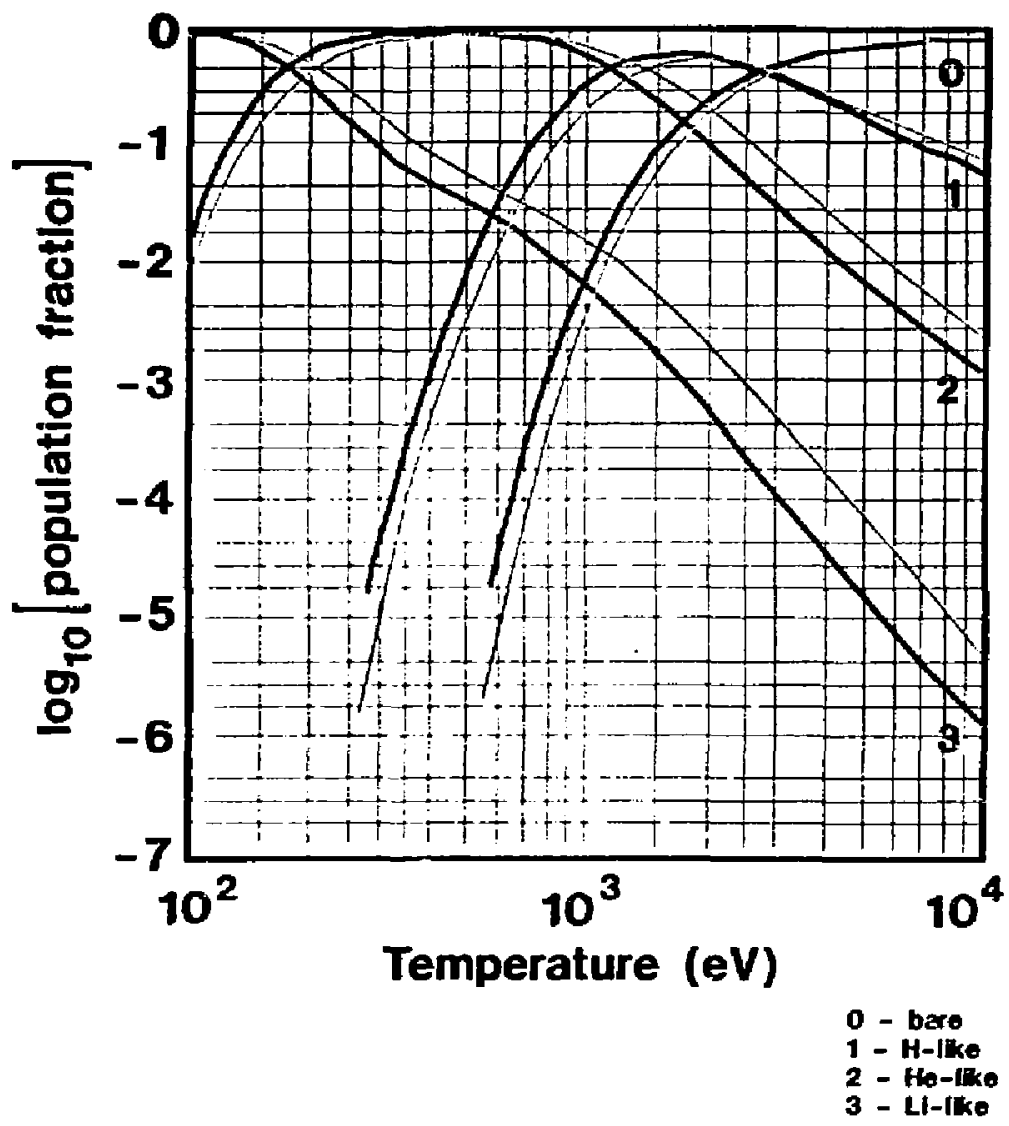

Effect of halt-strcingth rates of electron-collisional excitation/deexcitation. Populations of the four charge-states are computed by LINEZ both for the standard argon plasma (as in Fig. 2) and with all rate coefficients of e-collisional excitation/de-excitation multiplied by 0.5 . 


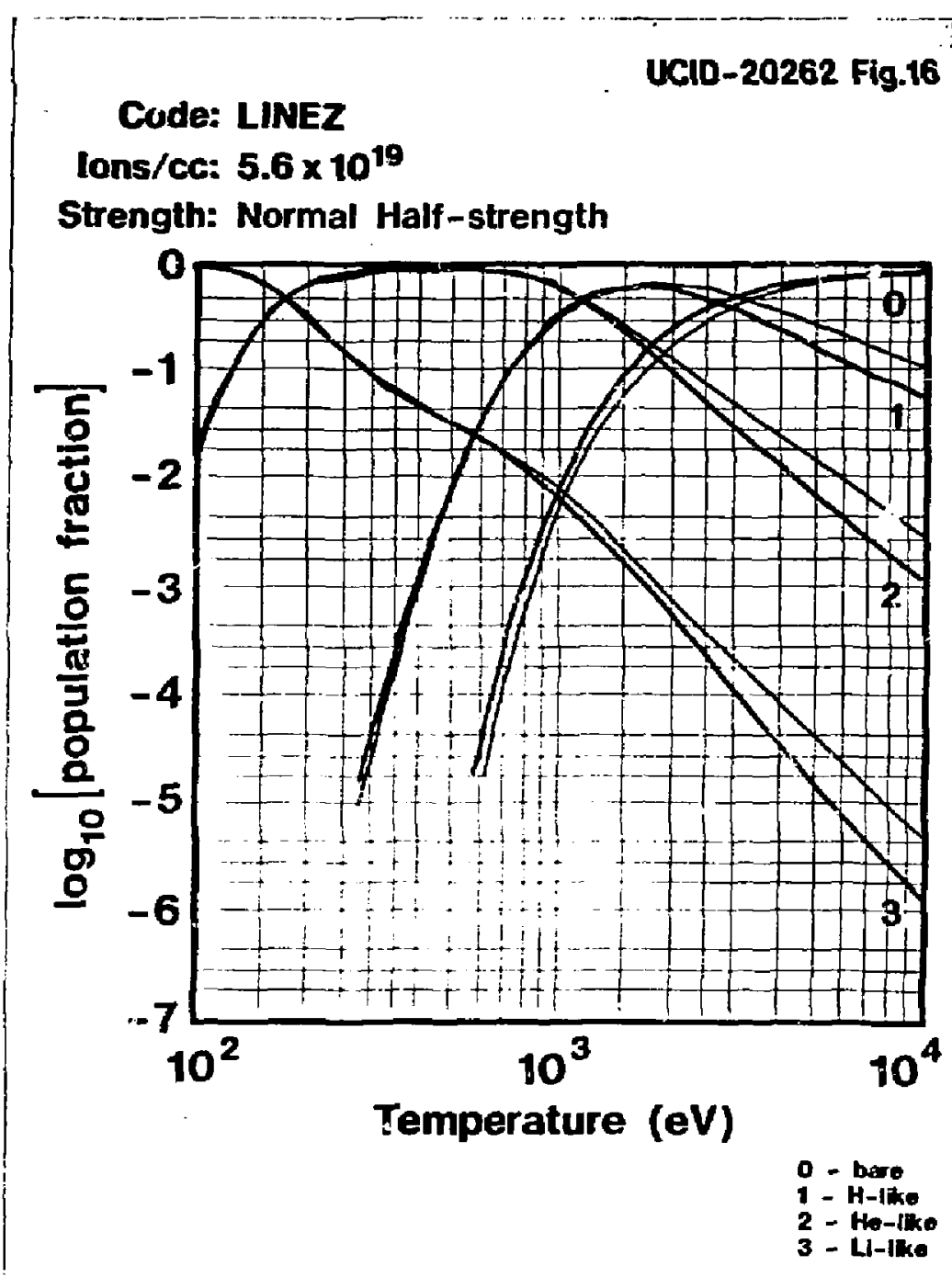

Effect of half-strength rates of electron-collisional ionization/? recombitation. Populations of the four charge-states are computed by LINEZ both for the standard Ar plasma (as in Fig. 2) and with all rate coefficients of e-coll. ionization and 3-body recombination multiplied by 0.5 . 
Code: LINEZ

UCID-20262 Fig. if

Ions/cC: $5.6 \times 10^{19}$

Strength: Normal No DR

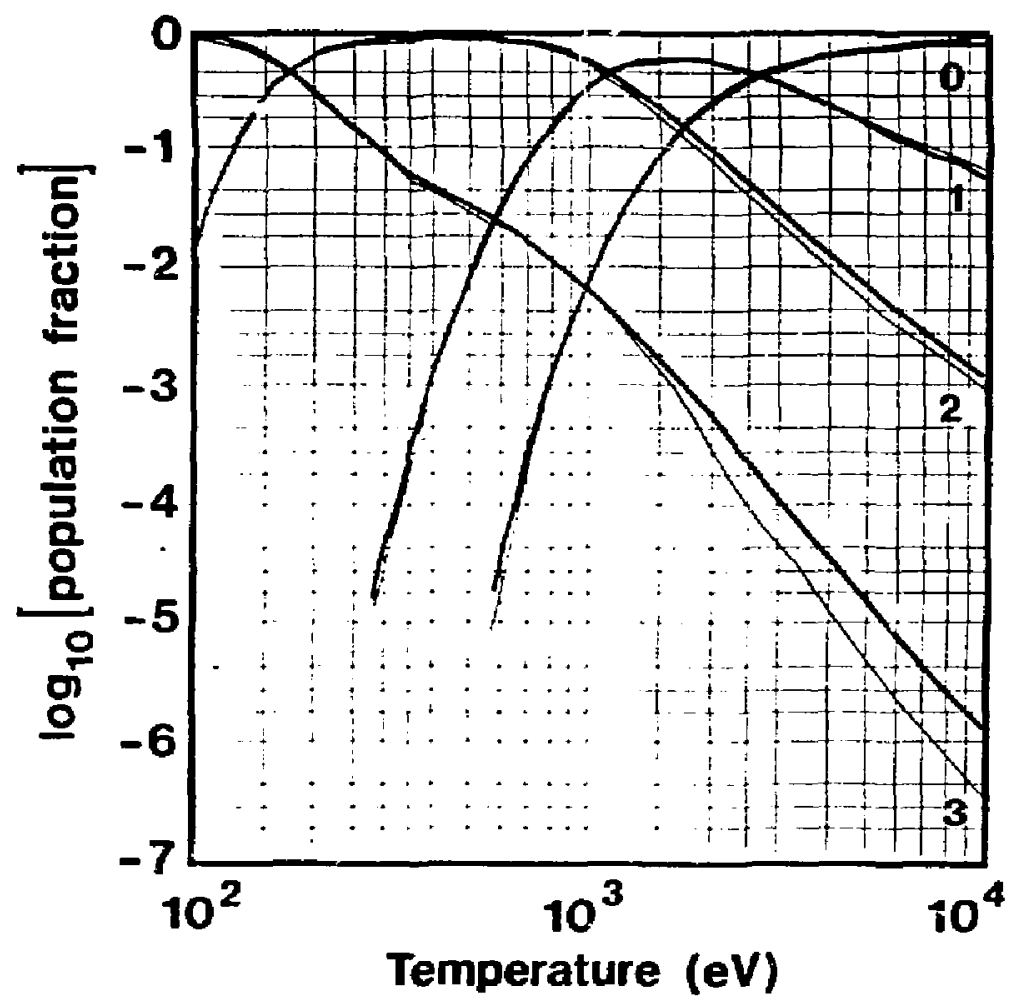

$$
\begin{aligned}
& 0 \text { - bare } \\
& 1 \text { - H-like } \\
& 2 \text { - He-lke } \\
& 3 \text { - Li-like }
\end{aligned}
$$

Effect of no dielectionic recombination. Populations of the four charge-states are computed by LINEZ both for the standard Ar plasma (as in Fig. 2) and with dielectronic recombination set to zero. 


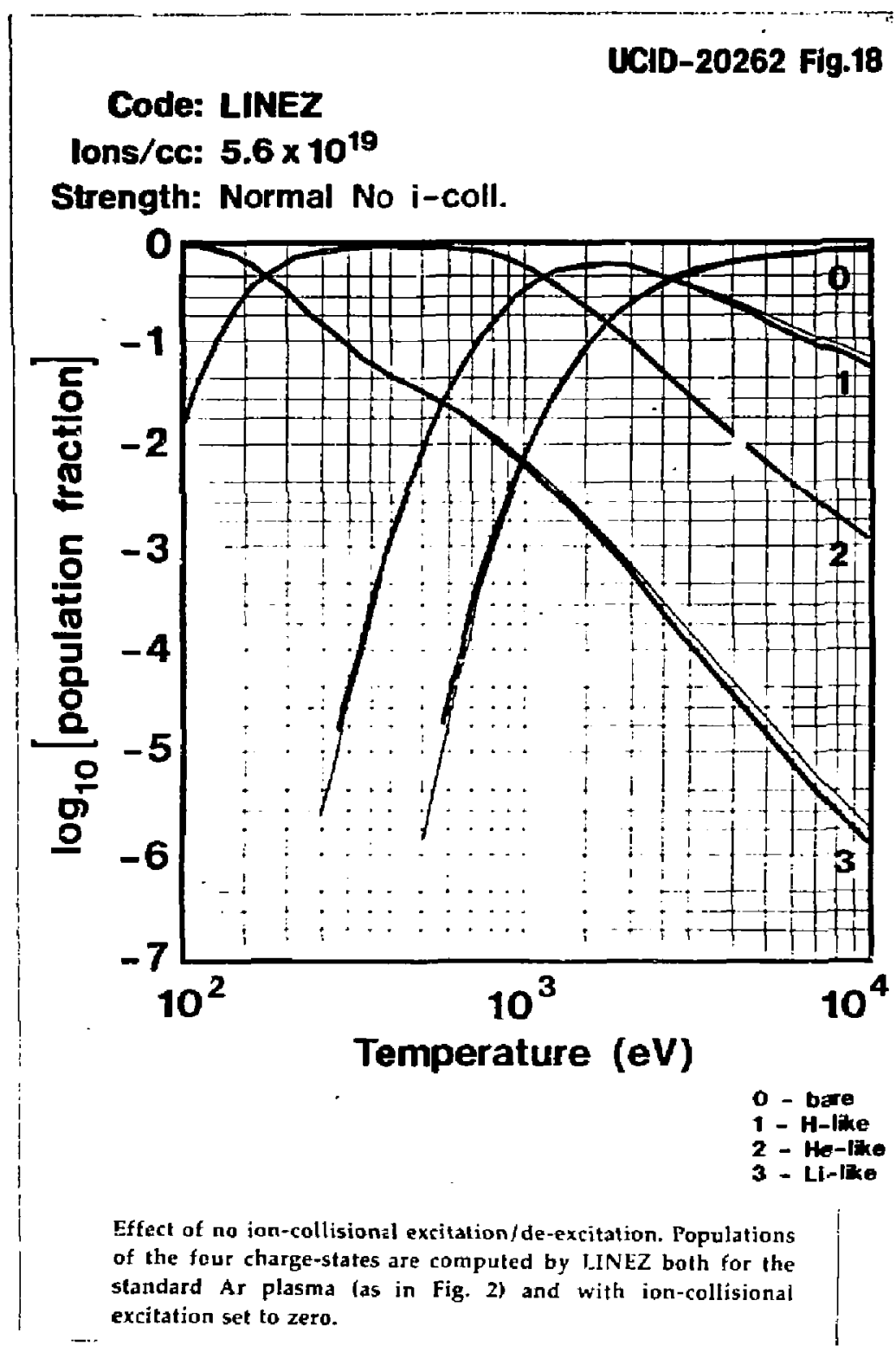


Code: LINEZ

lons/ce: $5.6 \times 10^{19}$

Strength: Normal $n_{\text {max }}=10$

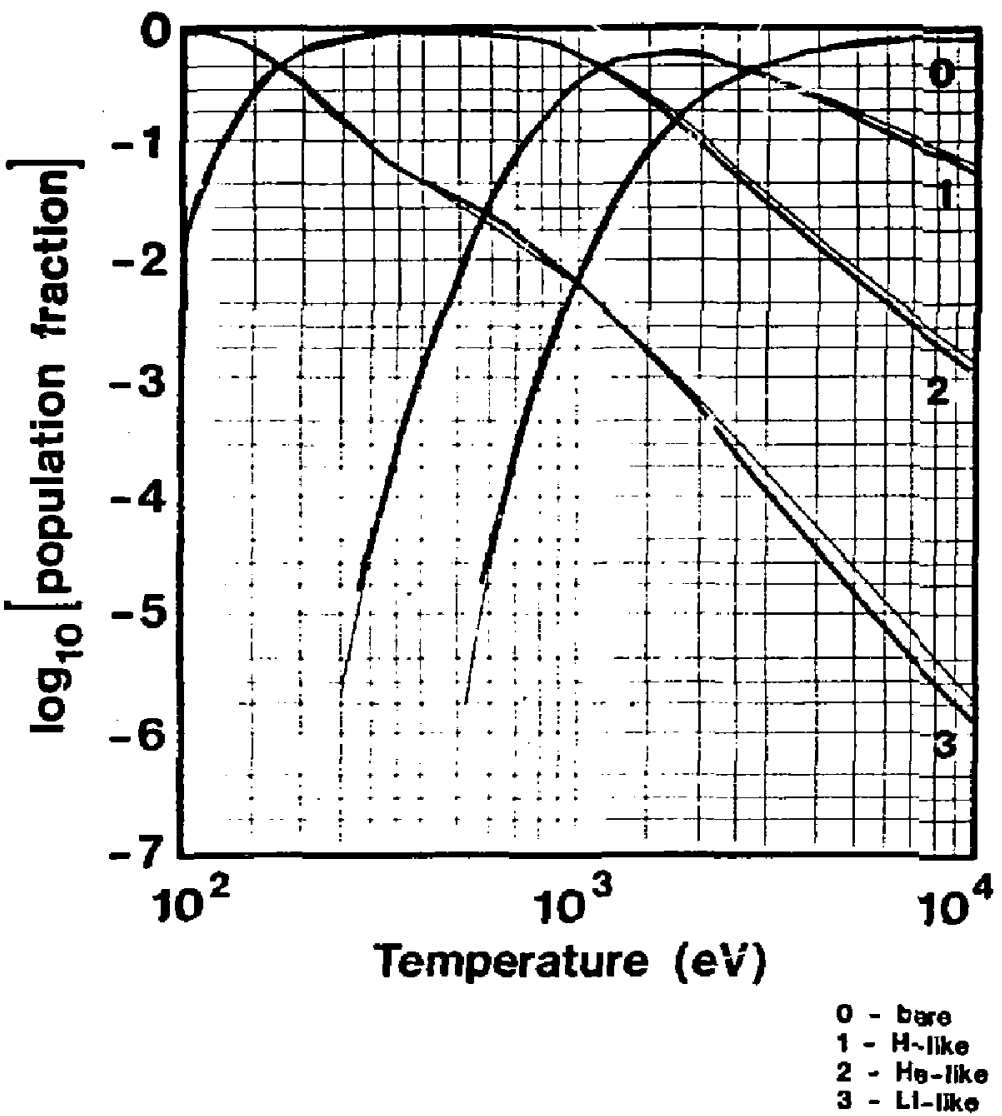

Effect of limiting the states to those having principal quantum: number $\leq n_{\max }=10$. Populations of the four charge-states are computed by LINEZ for the standard Ar plasma in two ways: (j) normal LINEZ, as in Fig. 2 , witt $n_{\max }$ set by the Bates criterion; ${ }^{11}$ and (ii) LINEZ with fixed $n_{m \leq x}=10$, plus Stewart-Pyatt continuum lowering. ${ }^{21}$ 
Code: LINEZ

UCID-20262 Fig.20

lons/cc: $5.6 \times 10^{19}$

Strength: Normal $n_{\text {max }}=5$

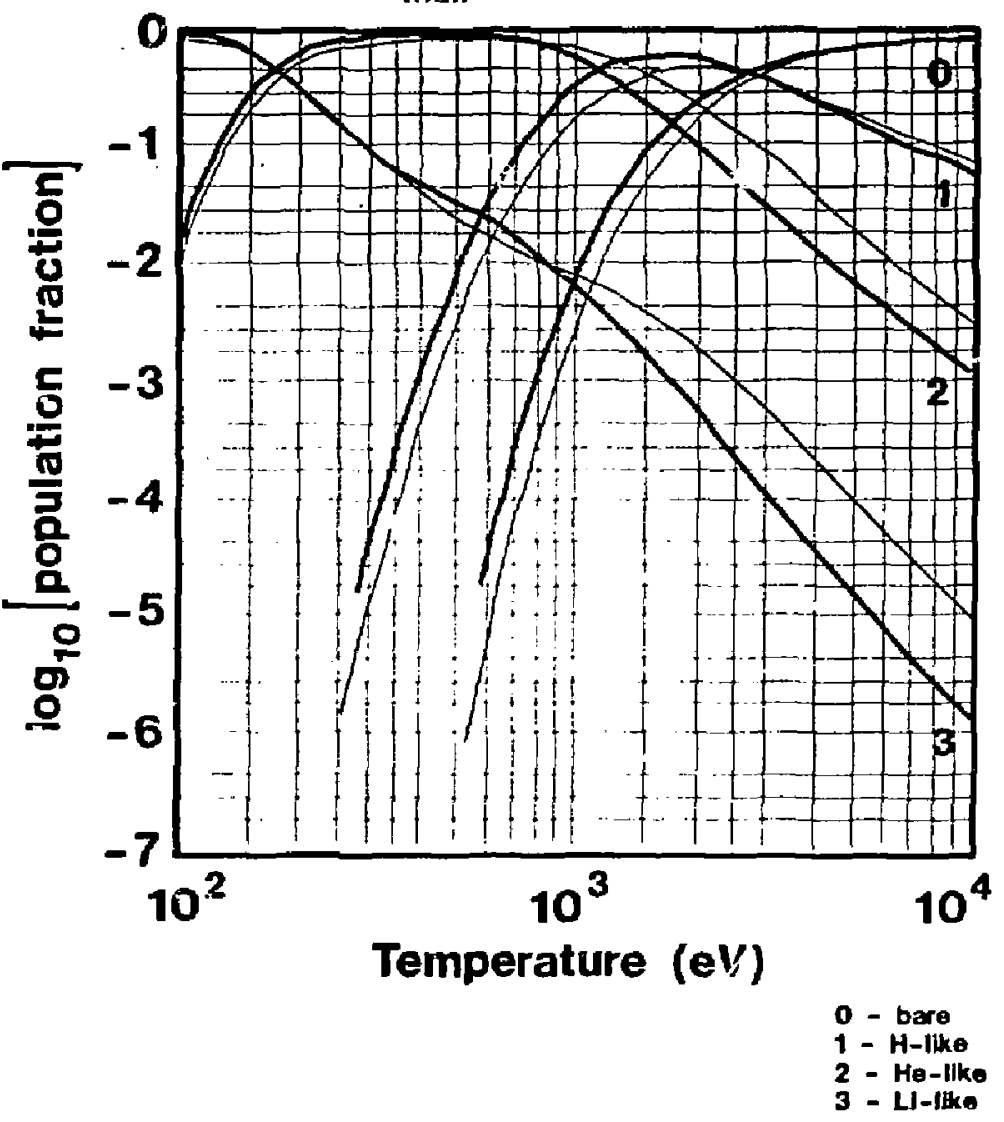

Effect of limiting the states to those having principal quantum numbers $\leq n_{\text {max }}=5$. Populations of the four charge-states are computed by LINEZ for the standard Ar piasma in two ways: (i) normal LINEZ, as in Fig. 2, with $\mathrm{t}_{\mathrm{m} \alpha \mathrm{x}}$ set by the Bates criterion; ${ }^{\prime \prime}$ and (ii) LINEZ with fixed $n_{\text {max }}=5$, plus Stewart-Pyatt continuum lowering. 


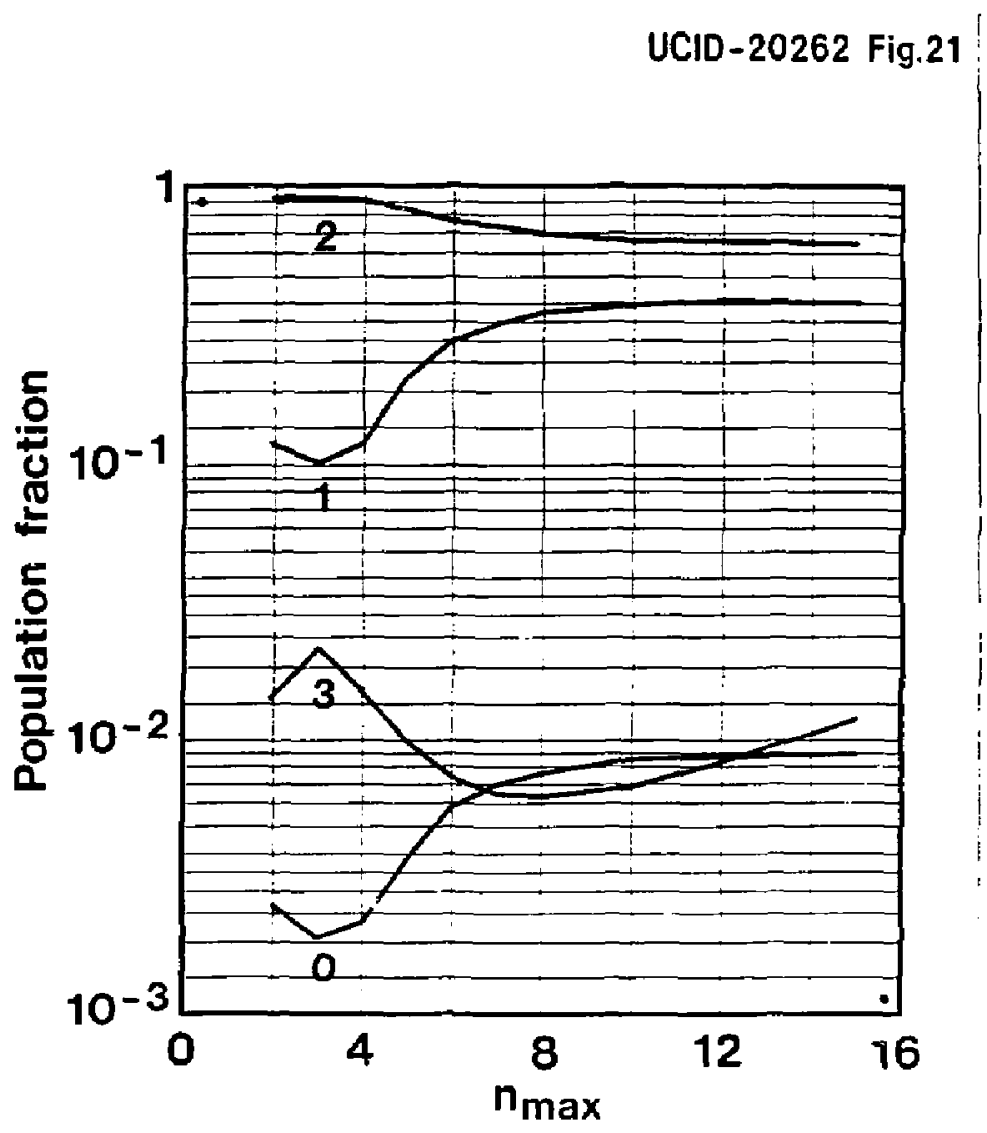

Effect of varying $n_{\max }$ in LINEZ. The optically thin Ar plasma is at the single case $1,000 \mathrm{eV}$ and $5.6 \times 10^{19}$ ions/cc. Line segments show LINEZ populations with fixed $n_{\max }$ and with Stewart-Pryatt continuum lowering. An overlay compares populations by the NLTE codes, including normal LINEZ, and lists the $\pi_{\text {mux }}$ used in each charge-state sy each code. 


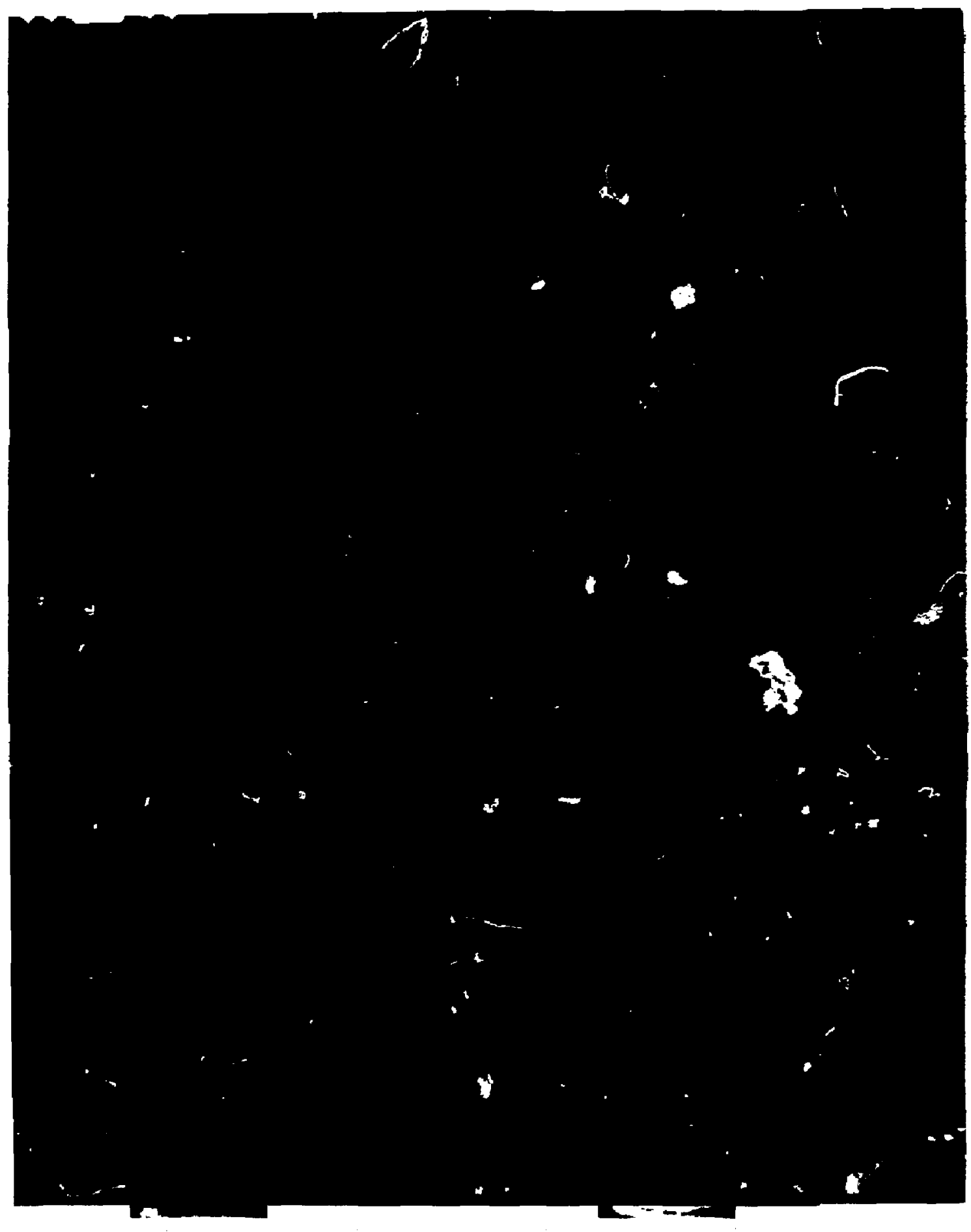




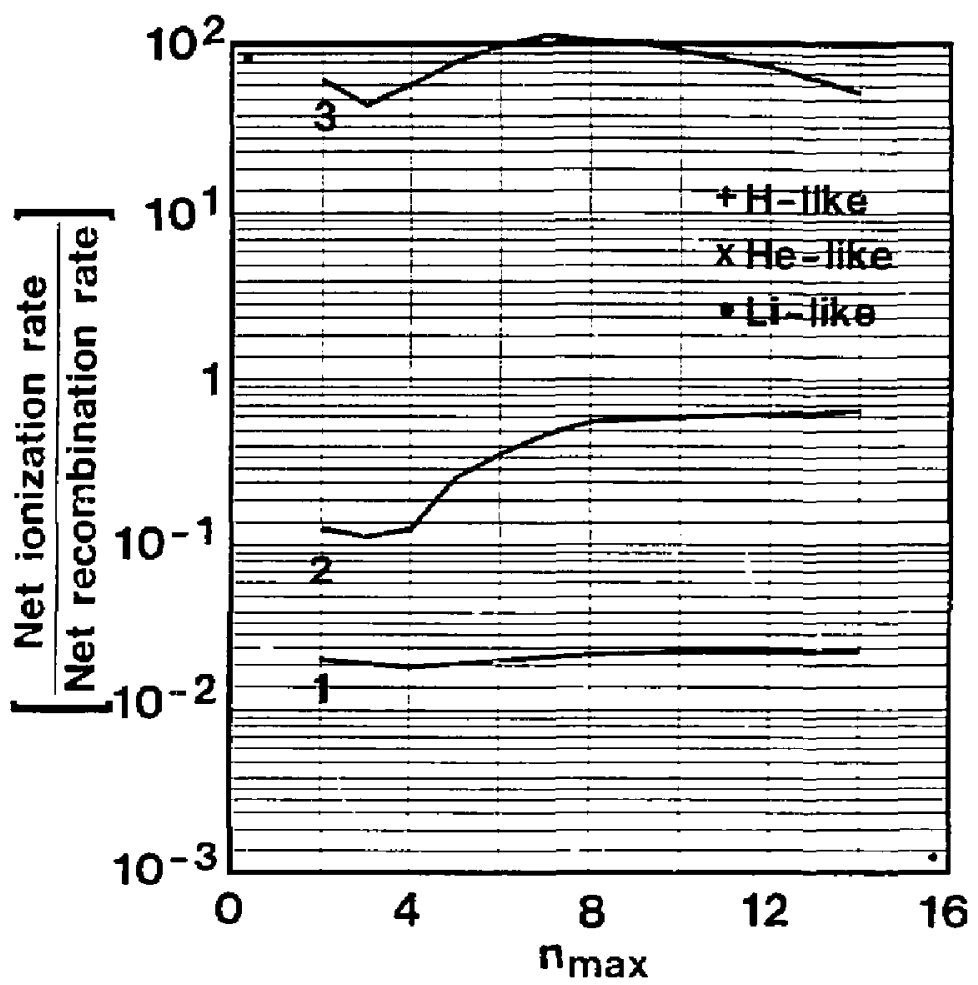

Effect of varying $\mathbf{n}_{\max }$ in LINEZ. Plotted are ratios [net ionization ratc] / lnet recombination rale] in the optically tíin Ar plasma for the single case, $1,000 \mathrm{eV}$ and $5.6 \times 10^{14}$ ions/cc. Lire segments show LINEZ ratios when $n_{\text {Inax }}$ is fixpd and with Slewart-Pyatt continuum lowering. An overlay compares the ratios by the NITE codes, including normal LINEZ, and lists the $n_{\text {mar }}$ used in cach charge-slate by each code. 


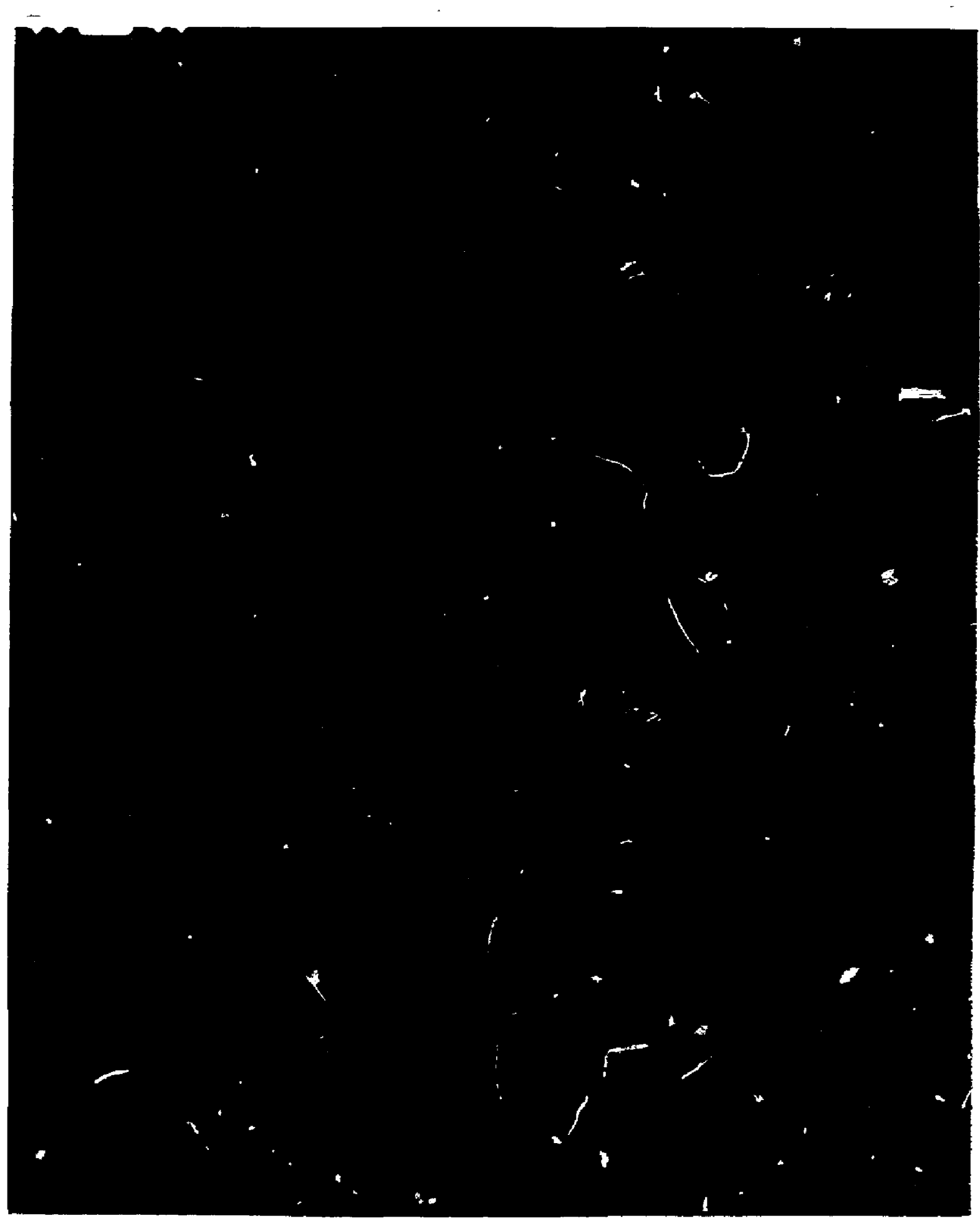




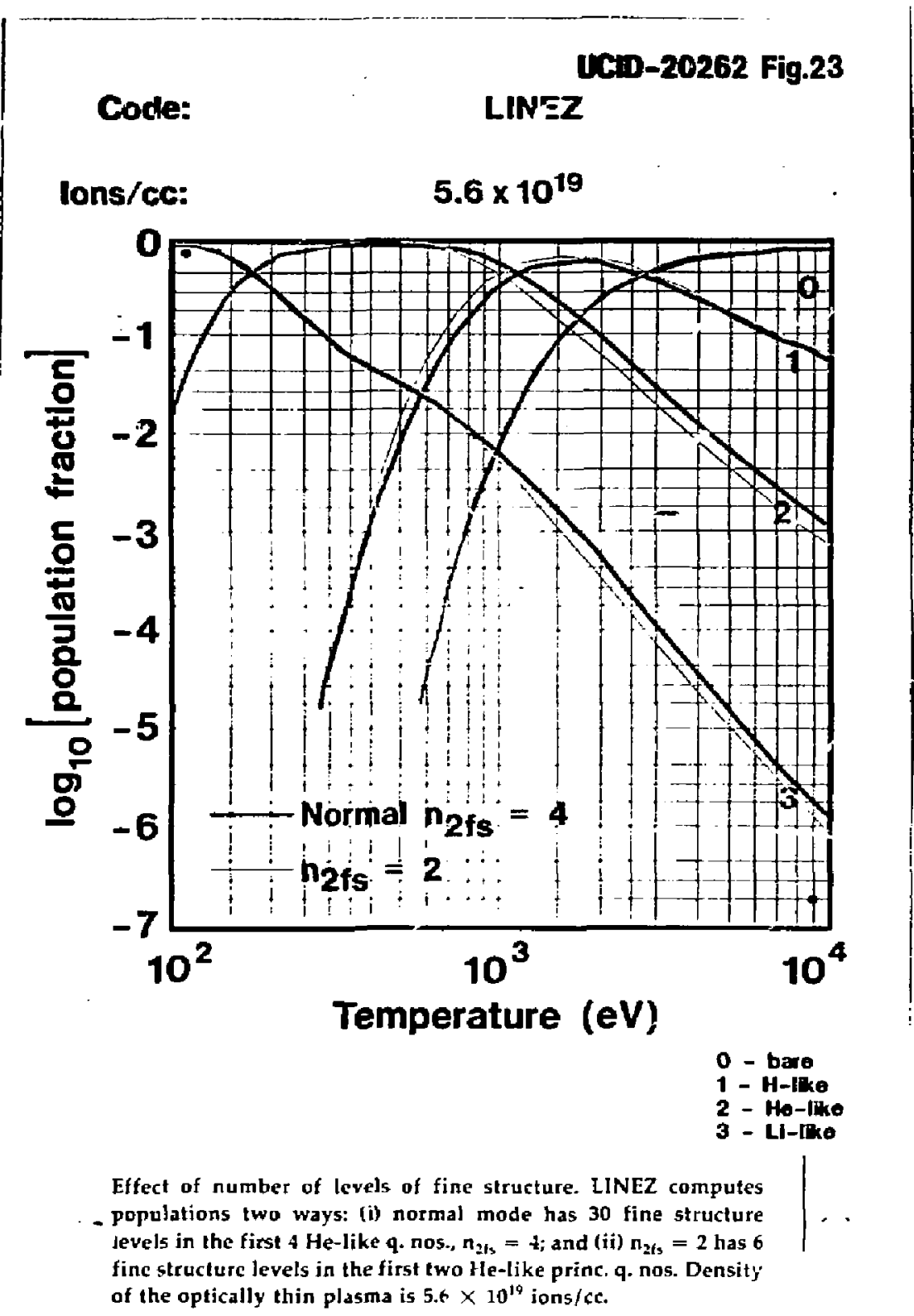

